\title{
IDŐJÁRÁS
}

Quarterly Journal of the Hungarian Meteorological Service

Vol. 124, No. 1, January-March, 2020, pp. 47-72

\section{Validation of the existing models for estimating diffuse solar radiation over Egypt}

\author{
S. M. Robaa \\ Astronomy, Space Sciences and Meteorology Department, \\ Faculty of Science, Cairo University, Giza - Egypt \\ Email:d_robaa@hotmail.com \\ (Manuscript received in final form February 7, 2019)
}

\begin{abstract}
The main objective of this study is to review and test the applicability of wellestablished models collected from the literature for estimating the monthly average daily diffuse solar radiation on a horizontal surface in Egypt. The different meteorological data measured at eight stations during the period 1987-2016 were used to calculate the monthly mean values of diffuse solar radiation over these stations using the collected models. The selected eight stations measure diffuse solar radiation component and have been chosen to cover the whole of Egypt. The collected models (fourteen models) were compared on the basis of many statistical error tests such as the relative percentage error, $(e \%)$, mean percentage error $(M P E)$, mean bias error $(M B D)$, root mean square error (RMSE), $t$-test, and Nash-Sutcliffe equation (NSE). According to the results, the Tarhan and Sarı model (Model 12) showed the best estimation of the diffuse solar radiation on a horizontal surface for all of the eight stations, and therefore, it is recommended for predicting diffuse solar radiation at any location in Egypt.
\end{abstract}

Key-words: solar energy, diffuse solar radiation, sunshine duration, extraterrestrial radiation, solar radiation models, model comparison, Egypt.

\section{Introduction}

Knowledge of local solar radiation components is essential in the design and study of many solar energy applications ( $L u$ et al, 1998; Li and Lam, 2000; Wong and Chow, 2001; Driesse and Thevenard, 2002; Almorox and Hontoria, 2004; AlMohamad, 2004; Kumar and Umanand, 2005). Although Egypt is a vast country and has abundant solar energy, solar radiation measurements are not easily 
available in Egypt (especially the diffuse solar radiation) because of not being able to afford the measuring equipments and techniques involved (Ibrahim, 1985). Therefore, it is important to develop methods to estimate the solar radiation on the basis of the more readily available meteorological data. Several models have been developed to estimate the amount of global solar radiation on horizontal surfaces in Egypt (Ibrahim, 1985; Sabbagh, 1977; El-Shahawy, 1984; El-Shazly, 1998; Trabea and Shaltout, 2000; Darwish and Taha, 2000; Tadros, 2000; ElMetwally, 2004 and 2005; El-Sebaii, and Trabea, 2005; Khalil and Shaffie, 2013; El-Metwally and Wald, 2013; Khalil and Shaffie, 2016). Unfortunately, the diffuse radiation measurements are very rare in Egypt, and there are no researches, except for the study of El-Sebaii and Trabea (2003), which made a concerning estimation of diffuse solar radiation in Egypt. Therefore, the main objective of this paper is to validate the best available models that predict the monthly mean daily diffuse radiation on a horizontal surface against an independent data set over Egypt, and thus, to select the most accurate model. All the most accurate empirical models which are used to estimate diffuse solar radiation, $D$, have been collected from literatures to evaluate the applicability of these models to estimate $D$ over different stations in Egypt. The collected models were compared on the basis of many statistical error tests.

\section{Comparison of models with literature}

The most accurate empirical models concerning estimation of diffuse solar radiation collected from the literature are as follows:

Model 1 (Hawas and Muneer, 1984):

$$
\frac{D}{H}=1.35-1.6075\left(\frac{H}{H_{0}}\right),
$$

Model 2 (Ulgen and Hepbasli, 2009):

$$
\frac{D}{H_{0}}=0.1155-0.1958\left(\frac{H}{H_{0}}\right),
$$

Model 3 (Gopinathan, 1988):

$$
\frac{D}{H}=0.697-0.577\left(\frac{n}{N_{o}}\right),
$$

Model 4 (Jamil and Akhtar, 2017):

$$
\frac{D}{H}=0.2932-1.8655\left(\frac{H}{H_{0}}\right)-1.5114\left(\frac{n}{N_{o}}\right),
$$


Model 5 (Gopinathan, 1988):

$$
\frac{D}{H}=0.879-0.575\left(\frac{H}{H_{0}}\right)-0.323\left(\frac{n}{N_{o}}\right),
$$

Model 6 (El-Sebaii et al. 2010):

$$
\frac{D}{H_{0}}=3.0020-3.8820\left(\frac{H}{H_{0}}\right)-0.1500\left(\frac{n}{N_{o}}\right),
$$

Model 7 (El-Sebaii and Trabea, 2003):

$$
\frac{D}{H}=-0.209+2.183\left(\frac{n}{N_{o}}\right)-1.785\left(\frac{n}{N_{o}}\right)^{2}
$$

Model 8 (Tarhan and Sarl, 2005):

$$
\frac{D}{H}=0.9885-1.4276\left(\frac{H}{H_{0}}\right)+0.5679\left(\frac{H}{H_{0}}\right)^{2},
$$

Model 9 (Jamil and Akhtar, 2017):

$$
\frac{D}{H}=0.3116+1.8043\left(\frac{H}{H_{0}}\right)+0.0501\left(\frac{H}{H_{0}}\right)^{2}-1.5118\left(\frac{n}{N_{o}}\right),
$$

Model 10 (Jamil and Akhtar, 2017):

$$
\frac{D}{H}=0.3017-1.8726\left(\frac{H}{H_{0}}\right)-1.5454\left(\frac{n}{N_{o}}\right)+0.0212\left(\frac{n}{N_{o}}\right)^{2},
$$

Model 11 (Jamil and Akhtar, 2017):

$$
\frac{D}{H_{0}}=-0.1776+1.6206\left(\frac{H}{H_{0}}\right)-0.6843\left(\frac{n}{N_{o}}\right)-0.2136\left(\frac{n}{N_{o}}\right)^{2},
$$

Model 12 (Tarhan and Sarl, 2005):

$$
\frac{D}{H}=1.0207-1.6582\left(\frac{H}{H_{0}}\right)+1.1018\left(\frac{H}{H_{0}}\right)^{2}-0.4019\left(\frac{H}{H_{0}}\right)^{3},
$$

Model 13 (Aras et al. 2006):

$$
\frac{D}{H}=1.7111-4.9062\left(\frac{H}{H_{0}}\right)+6.6711\left(\frac{H}{H_{0}}\right)^{2}-3.9235\left(\frac{H}{H_{0}}\right)^{3},
$$

Model 14 (Jamil and Akhtar, 2017):

$$
\frac{D}{H}=0.2191+2.3964\left(\frac{H}{H_{0}}\right)-0.3877\left(\frac{H}{H_{0}}\right)^{2}-1.7828\left(\frac{n}{N_{o}}\right)+0.1705\left(\frac{n}{N_{o}}\right)^{2},
$$


where $D$ is the monthly average of the daily diffuse solar radiation, $H$ is the monthly average of the daily global solar radiation, $H_{o}$ is the monthly average daily extraterrestrial radiation, $n$ is the day length, and $N_{o}$ is the maximum possible sunshine duration. $H_{o}$ was calculated from the following equation (Duffie, 1991):

$$
H_{o}=\frac{24}{\pi} I_{s} f\left(\cos \varphi \cos \delta \sin w+\frac{2 \pi}{360} w \sin \varphi \sin \delta\right),
$$

where $I_{S}$ is the solar constant $\left(=1367 \mathrm{Wm}^{-2}\right), f$ is the eccentricity correction factor of the Earth's orbit, $\varphi$ is the latitude of the site, $\delta$ is the sun declination, and $w$ is the mean sunrise hour angle for the given month. $f, \delta, w$, and $N_{o}$ can be computed by the following equations (Duffie, 1991):

$$
\begin{gathered}
f=\left(1+0.033 \cos \frac{360 n^{\prime}}{365}\right), \\
\delta=23.45 \sin \left[\frac{360\left(284+n^{\prime}\right)}{365}\right], \\
w=\cos ^{-1}(-\tan \varphi \tan \delta), \\
N_{o}=2 w / 15
\end{gathered}
$$

where $n^{\prime}$ is the day of the year.

\section{Data and comparison methods}

In this study, monthly mean values of global solar radiation and sunshine hours measured at of eight stations during the period 1987-2016 have been obtained from the Egyptian Meteorology Authority (EMA) to calculate the diffuse solar radiation, $D$ over these stations using the above corresponding models. Table 1 gives the list of the stations and their coordinates in addition to the type of the measured radiation at each station and its date of commencement of records. The monthly mean values of extraterrestrial solar radiation, $H_{o}$, and the day length, $n$, were calculated for each month of the year and for each station using Eqs. (15$19)$, and they were then employed to estimate $D$ for each station. 
Table 1. Coordinates of the Egyptian radiation measurements network and the radiation components measured together with the date of commencement of recording

\begin{tabular}{lcccccccc}
\hline \hline \multicolumn{1}{c}{ Station } & $\begin{array}{c}\text { Latitude } \\
(\mathbf{N})\end{array}$ & $\begin{array}{c}\text { Longitude } \\
(\mathbf{E})\end{array}$ & $\begin{array}{c}\text { Elevation } \\
(\mathbf{m})\end{array}$ & \multicolumn{4}{c}{ Measurement } & $\begin{array}{c}\text { Date of } \\
\text { commencement } \\
\text { of records* }\end{array}$ \\
\hline \hline Sidi-Barrani & $31^{\circ} 38^{\prime}$ & $25^{\circ} 24^{\prime}$ & 27 & $\mathrm{X}$ & $\mathrm{X}$ & - & $\mathrm{X}$ & 1984 \\
Matruh & $31^{\circ} 20^{\prime}$ & $27^{\circ} 13^{\prime}$ & 38 & $\mathrm{X}$ & $\mathrm{X}$ & - & $\mathrm{X}$ & $1961(1981)$ \\
El-Arich & $31^{\circ} 05^{\prime}$ & $33^{\circ} 49^{\prime}$ & 32 & $\mathrm{X}$ & $\mathrm{X}$ & - & $\mathrm{X}$ & 1980 \\
Tahrir & $30^{\circ} 39^{\prime}$ & $30^{\circ} 42^{\prime}$ & 16 & $\mathrm{X}$ & $\mathrm{X}$ & - & $\mathrm{X}$ & $1960(1981)$ \\
Cairo & $30^{\circ} 05^{\prime}$ & $31^{\circ} 17^{\prime}$ & 36 & $\mathrm{X}$ & $\mathrm{X}$ & $\mathrm{X}$ & $\mathrm{X}$ & $1969(1974)$ \\
Qena & $26^{\circ} 03^{\prime}$ & $32^{\circ} 12^{\prime}$ & 96 & $\mathrm{X}$ & $\mathrm{X}$ & - & $\mathrm{X}$ & 1979 \\
El-Kharga & $25^{\circ} 27^{\prime}$ & $30^{\circ} 32^{\prime}$ & 78 & $\mathrm{X}$ & $\mathrm{X}$ & - & $\mathrm{X}$ & $1964(1981)$ \\
Aswan & $23^{\circ} 58^{\prime}$ & $32^{\circ} 47^{\prime}$ & 192 & $\mathrm{X}$ & $\mathrm{X}$ & - & $\mathrm{X}$ & $1972(1981)$ \\
\hline
\end{tabular}

* The year in brackets indicates the data of commencement of diffuse and/or direct solar radiation records.

$G$ is the global solar radiation; $D$ is the diffuse solar radiation,

$I$ is the direct solar radiation, and $S$ is the sunshine duration.

The calculated values of diffuse solar radiation, $D_{c}$, were compared with the corresponding mean measured values, $D_{m}$ (mean of the period 1987-2016) in each model. Moreover, the performance of the models was also evaluated on the basis of the following statistical error tests: relative percentage error $(e \%)$, mean percentage error (MPE), mean bias error (MBE) root mean square error (RMSE), t-statistic (t), and Nash-Sutcliffe equation (NSE). $e \%, M P E, M B E, R M S E$, $t$ and NSE are defined by Equations (20-25), respectively, as below (Tiba, 2001; Ulgen and Hepbasli, 2003 and 2004; Notton et al. 2004; Soares et al. 2004; Tymvios et al. 2005; Mediavilla et al. 2005; Ulgen and Hepbasli, 2002; Togrul and Togrul, 2002; Stone, 1993; Chen et al., 2004):

$$
\begin{gathered}
e=\left[\left(D_{i, m}-D_{i, c}\right) / D_{i, m}\right]^{*} 100, \\
M P E=\sum_{i=1}^{N} \frac{\left[\left(D_{i, m}-D_{i, c}\right) / D_{i, m}\right] * 100}{N}, \\
M B E=\sum_{i=1}^{N} \frac{\left(D_{i, m}-D_{i, c}\right)}{N},
\end{gathered}
$$




$$
\begin{gathered}
R M S E=\left(\frac{\sum_{i=1}^{N}\left(D_{i, m}-D_{i, c}\right)}{N}\right)^{0.5}, \\
t=\left(\frac{(n-1) M B E^{2}}{R M S E^{2}-M B E^{2}}\right)^{0.5}, \\
N S E=1-\frac{\sum_{i=1}^{N}\left(\boldsymbol{D}_{i, m}-\boldsymbol{D}_{i, c}\right)^{2}}{\sum_{i=1}^{N}\left(\boldsymbol{D}_{i, m}-\overline{\boldsymbol{D}}_{i, c}\right)^{2}}
\end{gathered}
$$

where $D_{i, m}$ and $D_{i, c}$ are the $i$ th measured and calculated values of diffuse solar radiation, respectively, while $N$ is the number of observations taken into account.

\section{Results and discussion}

The values of monthly mean daily diffuse solar radiation intensity estimated using the above fourteen models (1-14) were compared with the corresponding measured values at the used eight stations. The relative percentage errors, $e(\%)$, between the estimated and measured values of the monthly mean daily diffuse solar radiation intensity were determined using Eq. (20) for the 12 months of the year. The statistical tests of MPE, MBE, RMSE, t-test, and NES were also calculated using Eqs. (21-25), respectively. The results are given in Tables 2-9. Furthermore, Table 10 summarizes the maximum and minimum values of the statistics errors, MPE, MBE, RMSE, $t$-test, and NSE, of each fourteen models at the eight selected stations.

It can be seen that the estimated values of $D_{c}$ at each station are in favorable agreement with the measured values $D_{m}$ for all the months of the year (Tables 2-9), whereas the percentage errors, $e(\%)$, for a single month not reaches $\pm 10 \%$ for any of the locations. Based on all statistical test results of MPE, MBE, RMSE, t-test, and NES (Tables 2-10), all models are recommended for using to estimate the diffuse radiation at all stations, whereas all statistical test results are in the range of acceptable values between $(-0.49$ and +5.12$)$ for MPE; $(-0.39$ and +2.78$)$ for MBE; $(+0.22$ and +3.51$)$ for RMSE; $(+0.03$ and +7.96$)$ for $t$-test; and $(0.9502$ and 0.9999) for NES. On the other hand, it was found that the Tarhan and Sar1 model, (Model 12), shows the best results among the all models for all of the stations. This is due to the fact that Model (12) has the lowest MPE, MBE, RMSE, 
and $t$-test value and highest $N E S$ values compared to the other fourteen models. It was found that, the overall percentage error, $e \%$, of Model (12) is in the range of acceptable values between -5.04 and $+3.31 \%$ with the lowest mean percentage error $(M P E)$ values that range from $-0.49 \%$ to $+0.27 \%$. Furthermore, $95.8 \%$ of these values $(e \%)$ lie between -2.64 and +2.94 for Model (12). Also, the $M B E$ values of Model (12) are usually equal to zero or very close to zero, while the values of $t$-test range from +0.03 to 1.88 . Furthermore, Model (12) has the highest values of NES and closest to 1.0, whereas they range from 0.9956 to 0.9999 . These are considered excellent indicators in that the Tarhan and Sar1 model (Model 12) gives precise estimation for each station and all Egypt with acceptable errors. Although Model (13) is almost like Model (12), Model (13) has higher values of MPE, MBE, RMSE, and t-test and lower values of NES than of Model (13), (See Tables 2-9). Therefore, it can be concluded that the Tarhan and Sar1 model (Model 12) is extremely recommended for use to estimate diffuse solar radiation at any location in Egypt, i.e., Model (12) is the best model for estimating diffuse solar radiation on a horizontal surface over Egypt. 


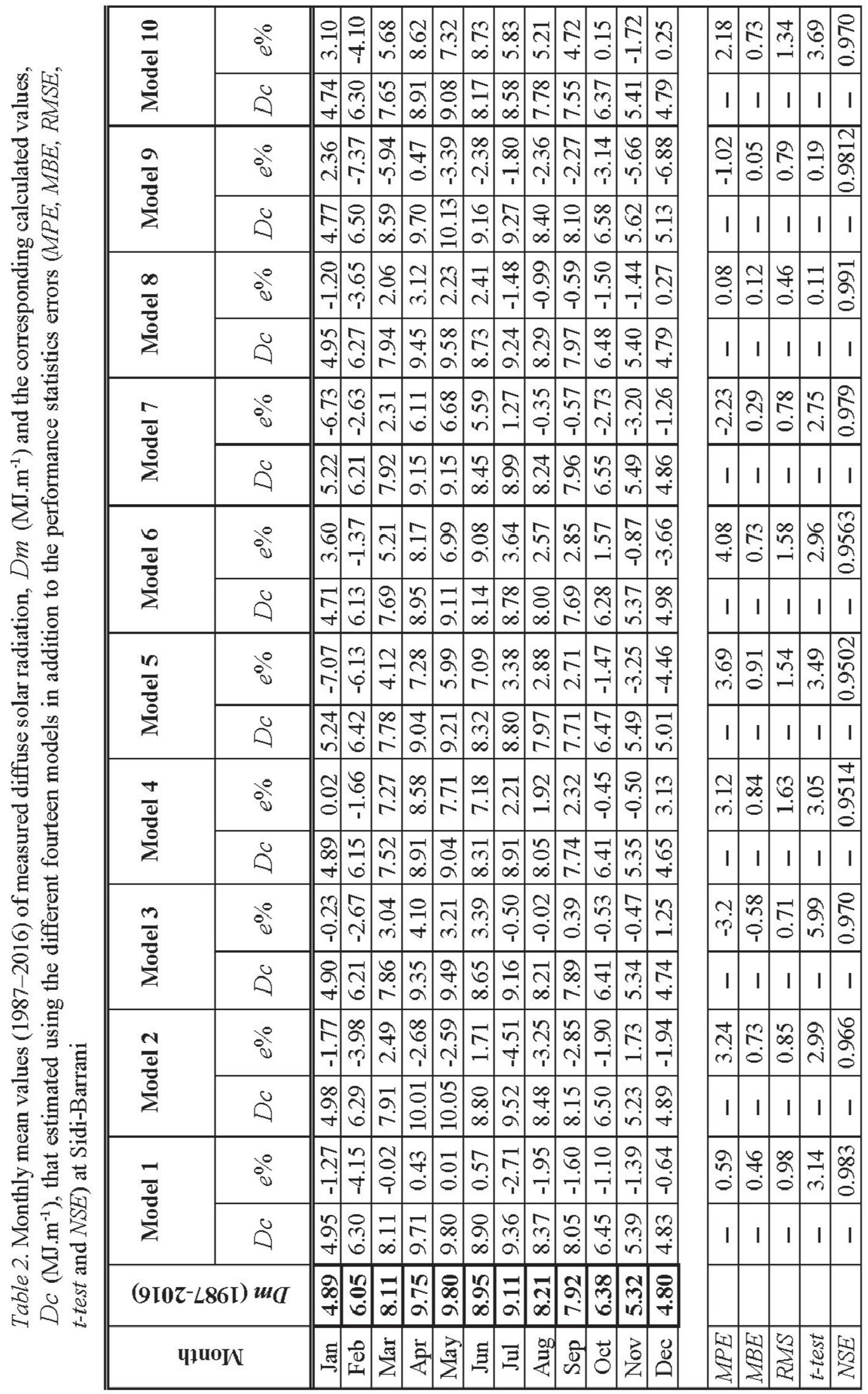




\begin{tabular}{|c|c|c|c|c|c|c|c|c|c|c|c|c|c|c|c|c|c|c|}
\hline \multirow{2}{*}{$\frac{ \pm}{\frac{D}{ \pm}}$} & oे & తి & 'ִ & 吕 & $\begin{array}{l}2 \\
2\end{array} \mid$ & $\frac{\Omega}{2}$ & ले & $\left|\begin{array}{c}\mathcal{Y} \\
\stackrel{\sim}{\sim}\end{array}\right|$ & $\stackrel{m}{i}$ & {$\left[\begin{array}{c}n \\
\sim \\
ن\end{array}\right.$} & 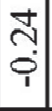 & $\begin{array}{l}\text { iे } \\
\text { i. } \\
\end{array}$ & $\begin{array}{c}\tilde{m} \\
m \\
m\end{array}$ & $\begin{array}{l}n \\
\hat{n} \\
\text { van }\end{array}$ & $\mid$\begin{tabular}{c}
0 \\
$\infty$ \\
\hdashline
\end{tabular} & తి & 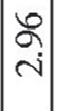 & مू \\
\hline & $\ddot{0}$ & $\begin{array}{l}\infty \\
\infty \\
\dot{\nabla}\end{array}$ & $\begin{array}{l} \pm \\
6 \\
6\end{array}$ & $\begin{array}{l}\stackrel{n}{2} \\
\end{array}$ & $\begin{array}{c}\stackrel{8}{\infty} \\
\infty\end{array} \mid$ & 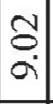 & 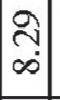 & $\begin{array}{c}\infty \\
\infty \\
\infty\end{array} \mid$ & $\begin{array}{c} \pm \\
0 \\
\infty\end{array}$ & $\frac{N}{2}$ & $\begin{array}{l}\text { f } \\
\dot{0}\end{array}$ & $\begin{array}{c}\tilde{m} \\
\dot{n}\end{array}$ & 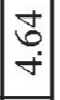 & 1 & 1 & 1 & 1 & 1 \\
\hline \multirow{2}{*}{$\frac{m}{\frac{0}{0}}$} & iे & D & 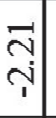 & 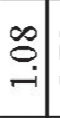 & $\left|\begin{array}{c}N \\
i n\end{array}\right|$ & $\begin{array}{c}2 \\
0 \\
\infty\end{array}$ & $\begin{array}{l}F \\
m \\
m\end{array}$ & $\left|\begin{array}{c}\infty \\
\hdashline \\
0\end{array}\right|$ & के & $\begin{array}{c}\stackrel{\overbrace{}}{0} \\
\text { } \\
\end{array}$ & $\begin{array}{c}0 \\
m \\
i \\
i\end{array}$ & $\begin{array}{c}+ \\
\infty \\
\\
\end{array}$ & 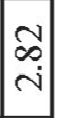 & $\frac{N}{0}$ & 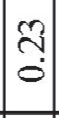 & 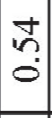 & 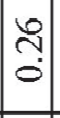 & 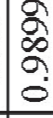 \\
\hline & $\stackrel{0}{0}$ & సે & $\begin{array}{l}\infty \\
\overrightarrow{6}\end{array}$ & $\begin{array}{c}\mathbb{1} \\
0 \\
\infty\end{array}$ & $\begin{array}{c}2 \\
-\end{array}$ & $\begin{array}{l}\overrightarrow{0} \\
\hat{\sigma}\end{array}$ & $\begin{array}{c}\overline{6} \\
\infty\end{array} \mid$ & $\left|\begin{array}{l}8 \\
8 \\
\hdashline\end{array}\right|$ & $\mid \begin{array}{l} \pm \\
m \\
\infty\end{array}$ & $\begin{array}{c}0 \\
\infty \\
\infty\end{array} \mid$ & $\frac{N}{0}$ & $\begin{array}{c}n \\
n \\
i n\end{array}$ & $\left|\begin{array}{l}0 \\
\hdashline \\
+\end{array}\right|$ & 1 & 1 & 1 & 1 & 1 \\
\hline \multirow{2}{*}{ 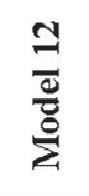 } & $\stackrel{\circ}{\circ}$ & 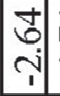 & 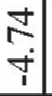 & m. & $\begin{array}{l}5 \\
\mathrm{i}\end{array}$ & $\begin{array}{c}0 \\
-1 \\
-i\end{array}$ & $\begin{array}{c}\bar{m} \\
\dot{r}\end{array}$ & $\begin{array}{l}0 \\
\vdots \\
0\end{array}$ & $\begin{array}{l}\infty \\
0 \\
0\end{array}$ & $\stackrel{?}{\circ}$ & \begin{tabular}{c}
$\infty$ \\
0 \\
\hdashline \\
\end{tabular} & $\frac{\sigma}{-1}$ & $\begin{array}{c}0 \\
0 \\
\end{array}$ & $\begin{array}{c}5 \\
0 \\
0\end{array}$ & $\begin{array}{l}8 \\
0 \\
0\end{array}$ & $\begin{array}{c}m \\
0 \\
0\end{array}$ & $\mid \begin{array}{l}3 \\
0 \\
0\end{array}$ & \\
\hline & $\stackrel{\Delta}{\Delta}$ & $\begin{array}{l}\text { s } \\
\text { ñ. }\end{array}$ & $\begin{array}{l}\tilde{m} \\
\text { bे }\end{array}$ & $\begin{array}{c}8 \\
\infty \\
\infty\end{array} \mid$ & \begin{tabular}{c}
$\infty$ \\
\multirow{+}{*}{} \\
$\sigma$
\end{tabular} & $\begin{array}{l}\hat{n} \\
\alpha\end{array}$ & $\left|\begin{array}{c}0 \\
6 \\
\infty\end{array}\right|$ & 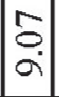 & $\begin{array}{l}n \\
\infty \\
\infty\end{array}$ & $\begin{array}{l}\infty \\
\infty \\
\infty \\
r\end{array} \mid$ & 告 & $\begin{array}{c}\stackrel{y}{+} \\
i\end{array}$ & $\mid \begin{array}{c}0 \\
\infty \\
\dot{\gamma}\end{array}$ & 1 & $\mathrm{I}$ & $\mathrm{I}$ & 1 & 1 \\
\hline \multirow{2}{*}{$\begin{array}{l}\exists \\
\frac{D}{8} \\
\frac{1}{2}\end{array}$} & o̊ & $\begin{array}{c}0 \\
0 \\
\end{array} \mid$ & $\begin{array}{l}J \\
0 \\
\dot{0}\end{array}$ & $\begin{array}{l}n \\
n \\
r\end{array}$ & $\begin{array}{l}+7 \\
r i\end{array}$ & $\begin{array}{c}6 \\
6 \\
1 \\
1\end{array}$ & $\begin{array}{c}F \\
i \\
i\end{array}$ & 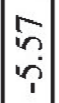 & $\begin{array}{c}\vec{m} \\
\dot{v}\end{array}$ & $\begin{array}{c}\bar{a} \\
\bar{r}\end{array}$ & \begin{tabular}{l}
$\curvearrowright$ \\
\multirow{i}{1}{}
\end{tabular} & $\begin{array}{l}2 \\
i \\
i\end{array}$ & $\begin{array}{c}8 \\
\dot{8} \\
\dot{1}\end{array} \mid$ & $\begin{array}{c}\infty \\
\alpha \\
r \\
1\end{array} \mid$ & $\left|\begin{array}{l}\infty \\
\infty \\
0 \\
0\end{array}\right|$ & $\begin{array}{l}2 \\
\infty \\
0\end{array}$ & $\frac{\wp}{-}$ & 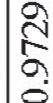 \\
\hline & $\stackrel{0}{0}$ & & $\begin{array}{l}\mathscr{0} \\
\dot{\Xi} \\
\text { in }\end{array}$ & \begin{tabular}{c|}
$\stackrel{P}{q}$ \\
$\infty$ \\
$\infty$
\end{tabular} & $\begin{array}{l}\overrightarrow{1} \\
\overrightarrow{0} \\
\end{array}$ & $\begin{array}{l}0 \\
\stackrel{1}{0} \\
\varrho\end{array}$ & 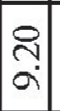 & $\left|\begin{array}{c}\sigma \\
\sigma \\
0\end{array}\right|$ & $\mid \begin{array}{c}0 \\
\infty \\
\infty\end{array}$ & $\begin{array}{c}\hat{3} \\
\infty\end{array}$ & ñ & $\begin{array}{c}f \\
\dot{f} \\
\text { in }\end{array}$ & $\begin{array}{l} \pm \\
\text { +े } \\
\end{array}$ & & & & & \\
\hline \multicolumn{2}{|c|}{$(9 \mathrm{I} 0 \mathrm{Z}-\angle 86 \mathrm{I}) u^{\prime} G$} & 命 & $\begin{array}{c}n \\
0 \\
0\end{array}$ & $\underset{\infty}{\vec{\infty}}$ & $\begin{array}{l}n \\
2 \\
2\end{array}$ & $\begin{array}{l}\varnothing \\
\infty \\
\propto\end{array}$ & $\begin{array}{l}2 \\
\infty \\
\infty\end{array} \mid$ & $=$ & $\underset{\infty}{-1}$ & $\begin{array}{l}\Omega \\
\end{array}$ & 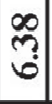 & 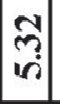 & $\begin{array}{c}\stackrel{\oplus}{\infty} \\
+ \\
+\end{array}$ & & & & & \\
\hline \multicolumn{2}{|c|}{ чриоК } & : & 0 & $\sum_{i}^{3}$ & 氞 & 궁 & $\Xi$ & $\Xi$ & 哭 & व्र & $\overline{0}$ & $\begin{array}{l}\vec{b} \\
\text { z } \\
\end{array}$ & $\begin{array}{l}0 \\
\stackrel{0}{0} \\
\end{array}$ & 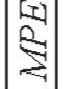 & $\mid \begin{array}{l}1 \\
02 \\
0 \\
-4\end{array}$ & & 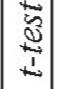 & 昜 \\
\hline
\end{tabular}




\begin{tabular}{|c|c|c|c|c|c|c|c|c|c|c|c|c|c|c|c|c|c|c|}
\hline \multirow{2}{*}{ 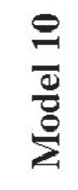 } & $g_{0}^{\circ}$ & $\begin{array}{l}? \\
0 \\
0\end{array}$ & - & $\begin{array}{l}\stackrel{8}{\infty} \\
+ \\
\end{array}$ & $\begin{array}{l}\stackrel{\partial}{+} \\
\text { ì }\end{array}$ & 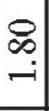 & $\begin{array}{l}\text { Nิ } \\
\text { in }\end{array}$ & 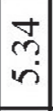 & $\stackrel{m}{\dot{m}}$ & $\begin{array}{c}n \\
m \\
m\end{array}$ & $\begin{array}{l}\hat{1} \\
\dot{1} \\
\end{array}$ & 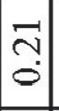 & $\bar{a}$ & సิ & $\begin{array}{c}\infty \\
0 \\
\dot{1}\end{array}$ & $\begin{array}{l}\infty \\
\stackrel{\circ}{\circ}\end{array}$ & $\begin{array}{l}\stackrel{2}{0} \\
\dot{N}\end{array}$ & 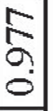 \\
\hline & $\stackrel{0}{0}$ & ? & $\begin{array}{l}\hat{a} \\
\text { na }\end{array}$ & $\stackrel{2}{2}$ & $\begin{array}{l}N \\
\infty \\
\infty \\
\infty\end{array}$ & $\begin{array}{l} \pm \\
\vdots \\
\vdots\end{array}$ & $\begin{array}{l}\Omega \\
\infty\end{array}$ & $\stackrel{m}{a}$ & $\stackrel{+}{\infty}$ & 7 & $\begin{array}{l}0 \\
? \\
6 \\
6\end{array}$ & $\begin{array}{c}\text { ते } \\
\text { in }\end{array}$ & $\stackrel{n}{+}$ & I & 1 & 1 & $\mathrm{I}$ & $\mathrm{I}$ \\
\hline \multirow{2}{*}{$\frac{a}{\frac{a}{0}}$} & oे & $\vec{m}$ & $\begin{array}{l}2 \\
\vdots \\
1\end{array}$ & $\begin{array}{l}6 \\
-6 \\
-1\end{array}$ & $\begin{array}{l}\widehat{\alpha} \\
\text { ते }\end{array}$ & $\begin{array}{l}\text { ஸ̂ } \\
\text { }\end{array}$ & $\vec{i}$ & $\begin{array}{l}n \\
i \\
i\end{array}$ & 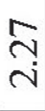 & 今ิ & \begin{tabular}{l} 
o \\
\hdashline \\
\end{tabular} & $\begin{array}{l}0 \\
\infty \\
0 \\
1\end{array}$ & $\underset{\sim}{\stackrel{\infty}{\sim}}$ & $\stackrel{n}{0}$ & กิ & $\begin{array}{l}n \\
6 \\
0 \\
0\end{array}$ & $\begin{array}{l}2 \\
0 \\
0\end{array}$ & 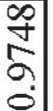 \\
\hline & $\stackrel{\Delta}{0}$ & $\stackrel{n}{\circ}$ & 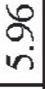 & $\begin{array}{c}\vec{N} \\
\infty\end{array}$ & $\begin{array}{l}8 \\
\circ \\
\circ\end{array}$ & : & $\stackrel{-}{a}$ & $\begin{array}{l}0 \\
\curvearrowright \\
\curvearrowright\end{array}$ & $\begin{array}{l}N \\
\infty \\
\infty\end{array}$ & $\begin{array}{l}8 \\
-1\end{array}$ & $\begin{array}{l}-6 \\
0 \\
0\end{array}$ & $\begin{array}{c}0 \\
\text { in } \\
\text { n' }\end{array}$ & $\begin{array}{l}0 \\
\text { ? } \\
\forall \\
\end{array}$ & $\mathrm{I}$ & $\mathrm{I}$ & 1 & $\mathrm{I}$ & $\mathrm{I}$ \\
\hline \multirow{2}{*}{$\frac{\infty}{\frac{\infty}{\delta}}$} & $o_{0}^{\circ}$ & $\begin{array}{l}\text { व } \\
\text { m. }\end{array}$ & $\begin{array}{l}\text { 寸 } \\
\dot{1}\end{array}$ & $\frac{n}{7}$ & 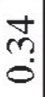 & $\vec{\sim}$ & $\begin{array}{l}0 \\
\stackrel{0}{0}\end{array}$ & $\stackrel{1}{\circ}$ & $\stackrel{\infty}{\stackrel{\infty}{\prime}}$ & $\begin{array}{c}\text { ñ? } \\
? \\
T\end{array}$ & 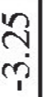 & $\begin{array}{c}2 \\
-1 \\
1\end{array}$ & $\begin{array}{l}J \\
\stackrel{\sim}{*}\end{array}$ & $\stackrel{\overbrace{}}{\stackrel{0}{0}}$ & 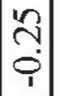 & $\stackrel{n}{0}$ & $\begin{array}{l}\bar{\infty} \\
\dot{0}\end{array}$ & $\begin{array}{l}\stackrel{2}{\circ} \\
\stackrel{0}{0}\end{array}$ \\
\hline & $\stackrel{0}{0}$ & $\exists=$ & & $\begin{array}{c}\infty \\
\stackrel{\infty}{\infty} \\
\infty\end{array}$ & ભి & mి & $\stackrel{\stackrel{\sim}{+}}{\circ}$ & $\begin{array}{l}\infty \\
\infty \\
\alpha\end{array} \mid$ & $\underset{\infty}{\stackrel{\infty}{\infty}}$ & $\left|\begin{array}{c}\infty \\
0 \\
\infty \\
\infty\end{array}\right|$ & $\begin{array}{l}\hat{\sigma} \\
0 \\
0\end{array}$ & $\begin{array}{c}1 \\
\text { in }\end{array}$ & mo & I & I & 1 & I & $\mathrm{I}$ \\
\hline \multirow{2}{*}{$\frac{\pi}{\frac{\sigma}{0}}$} & $o_{0}^{0}$ & ?] & 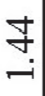 & $\stackrel{\overbrace{}}{\mathrm{i}}$ & ฉ & $\begin{array}{l} \pm \\
\text { ¿ } \\
\text { i }\end{array}$ & 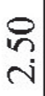 & $\begin{array}{l}\infty \\
\stackrel{\sim}{\sim}\end{array} \mid$ & $\stackrel{2}{\stackrel{2}{0}}$ & $\begin{array}{l}\searrow \\
\vdots \\
0\end{array}$ & $\begin{array}{l} \pm \\
\stackrel{ \pm}{2} \\
i\end{array}$ & $\begin{array}{l}\infty \\
\stackrel{\infty}{i} \\
ن\end{array} \mid$ & $\begin{array}{l}n \\
\infty \\
\dot{\gamma}\end{array}$ & $\frac{1}{-1}$ & $\stackrel{\Xi}{=}$ & $\stackrel{9}{\exists}$ & $\begin{array}{l}2 \\
0\end{array}$ & $\begin{array}{l}\hat{\partial} \\
\vdots \\
0\end{array}$ \\
\hline & $\stackrel{0}{0}$ & $\stackrel{\infty}{\circ}$ & $\begin{array}{l}\tilde{N} \\
\infty \\
\text { ri }\end{array}$ & $\begin{array}{c}0 \\
\infty \\
\infty\end{array}$ & $\stackrel{\nabla}{\sigma}$ & $\begin{array}{l}m \\
\infty \\
\sigma\end{array}$ & $\frac{a}{\sigma}$ & 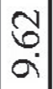 & $\stackrel{ \pm}{n}$ & পి & $\begin{array}{c}\infty \\
0 \\
0\end{array}$ & $\begin{array}{c}5 \\
\text { in }\end{array}$ & $\underset{\sim}{\stackrel{\sim}{*}}$ & I & 1 & $\mathrm{I}$ & 1 & 1 \\
\hline \multirow{2}{*}{$\frac{0}{\frac{0}{0}}$} & $\stackrel{0}{0}$ & ?2 & $\begin{array}{l}n \\
? \\
0\end{array}$ & $\begin{array}{l} \pm \\
i n\end{array}$ & ֶֶ & $\stackrel{\Omega}{\Omega}$ & $\begin{array}{l}6 \\
\dot{n}\end{array}$ & 吕 & $\widehat{\sigma}$ & $\begin{array}{l}n \\
n \\
1 \\
1\end{array}$ & $\begin{array}{l}\infty \\
\infty \\
\infty \\
i \\
?\end{array}$ & $\begin{array}{l}\infty \\
\stackrel{\infty}{+} \\
\stackrel{+}{2}\end{array}$ & $\begin{array}{l} \pm \\
\dot{r}\end{array}$ & $\stackrel{\substack{\sim \\
-i}}{\mid}$ & 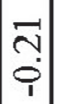 & $\stackrel{\circ}{\stackrel{2}{\circ}}$ & $\begin{array}{l}3 \\
\dot{r}\end{array}$ & $\begin{array}{l}\stackrel{\infty}{5} \\
\Omega \\
0\end{array}$ \\
\hline & $\stackrel{0}{0}$ & 学 & $\begin{array}{l}\hat{2} \\
\stackrel{n}{n}\end{array}$ & ลे & $\begin{array}{l}\stackrel{2}{\circ} \\
\stackrel{0}{0}\end{array}$ & $\begin{array}{c}\text { m. } \\
\text { aे }\end{array}$ & $\begin{array}{l}\mathscr{\Omega} \\
\infty\end{array}$ & $\begin{array}{l}\infty \\
6 \\
0 \\
0\end{array}$ & $\stackrel{n}{n}$ & $\begin{array}{c}m \\
0 \\
\infty \\
\infty\end{array}$ & $\begin{array}{l}0 \\
\infty \\
0 \\
0\end{array}$ & $\begin{array}{l}0 \\
i\end{array}$ & $\underset{\text { શे }}{+}$ & I & 1 & 1 & 1 & 1 \\
\hline \multirow{2}{*}{$\frac{\frac{n}{d}}{\frac{10}{0}}$} & $\stackrel{\circ}{\circ}$ & 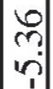 & $\begin{array}{l}\text { Na } \\
\\
i\end{array}$ & $\begin{array}{l}\infty \\
\infty \\
r\end{array}$ & ஒे & $\begin{array}{l}0 \\
m \\
n\end{array}$ & $\begin{array}{l}6 \\
6 \\
6\end{array}$ & $\begin{array}{l}\vec{j} \\
i\end{array}$ & $\begin{array}{l}\frac{6}{1} \\
i\end{array}$ & $\begin{array}{l}m \\
\vec{i}\end{array} \mid$ & $\stackrel{?}{?}$ & $\begin{array}{l}n \\
n \\
i\end{array}$ & $\begin{array}{c}m \\
\dot{m}\end{array}$ & $\begin{array}{l}2 \\
\mathrm{n}\end{array} \mid$ & $\left|\begin{array}{c}\mathbf{0} \\
\text { ì }\end{array}\right|$ & $\frac{m}{\overrightarrow{0}}$ & $\begin{array}{l}n \\
n \\
n\end{array}$ & $\begin{array}{l}8 \\
8 \\
8\end{array}$ \\
\hline & ڤั & ? & $\underset{ֶ}{N}$ & $\begin{array}{c}m \\
\infty \\
\infty\end{array}$ & $\underset{\infty}{\mathbb{\infty}}$ & $\begin{array}{l}\infty \\
\stackrel{\infty}{n} \\
a\end{array}$ & $\begin{array}{l}2 \\
\infty \\
\infty \\
\infty\end{array}$ & $\begin{array}{c}0 \\
n \\
\alpha\end{array}$ & $\stackrel{m}{n}$ & $\begin{array}{l}-\infty \\
\infty \\
\infty\end{array}$ & $\begin{array}{l}0 \\
n \\
0 \\
0\end{array}$ & $\begin{array}{l}\text { in } \\
\text { in }\end{array}$ & $\stackrel{?}{+}$ & I & 1 & 1 & 1 & 1 \\
\hline \multirow{2}{*}{$\frac{\frac{ \pm}{0}}{\frac{D}{E}}$} & 80 & $\begin{array}{l}0 \\
0 \\
1\end{array}$ & $\begin{array}{l}\stackrel{9}{+} \\
\underset{+}{+}\end{array}$ & $\begin{array}{l}\infty \\
n \\
m\end{array}$ & $\begin{array}{l}\hat{\sigma} \\
\text { in }\end{array}$ & $\begin{array}{l}\stackrel{8}{6} \\
+\end{array}$ & $\underset{\text { ì }}{\stackrel{ \pm}{ }}$ & $\underset{\sim}{ \pm}$ & $\stackrel{?}{\stackrel{9}{-}}$ & 古 & $\begin{array}{c}8 \\
\\
-1\end{array}$ & $\begin{array}{c}\infty \\
\infty \\
-1 \\
1\end{array}$ & $\stackrel{-\sigma}{-i}$ & 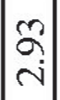 & $\stackrel{9}{\circ}$ & $\vec{n}$ & $\begin{array}{c}\stackrel{v}{ } \\
\sim \\
+\end{array}$ & $\begin{array}{l}\infty \\
\infty \\
\alpha \\
\alpha \\
\alpha\end{array}$ \\
\hline & $\stackrel{0}{0}$ & $\begin{array}{l}f \\
\dot{f}\end{array}$ & $\frac{7}{6}$ & $\begin{array}{l}2 \\
0 \\
\infty \\
\infty\end{array}$ & $\begin{array}{l}\infty \\
\infty \\
\infty \\
\infty\end{array}$ & $\begin{array}{l}n \\
0 \\
0 \\
0\end{array}$ & $\begin{array}{l} \pm \\
\infty \\
\infty\end{array}$ & $\begin{array}{l}8 \\
0 \\
0\end{array}$ & $\underset{\infty}{\mathscr{B}}$ & $\begin{array}{c}0 \\
\infty \\
r \\
r\end{array}$ & 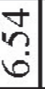 & $\begin{array}{c}n \\
m \\
n \\
n\end{array}$ & $\stackrel{\text { mे }}{+}$ & I & 1 & 1 & 1 & $\mathrm{I}$ \\
\hline \multirow{2}{*}{$\frac{m}{\frac{0}{0}}$} & oे & 웅. & $\begin{array}{l}m \\
\\
\\
\end{array}$ & $\begin{array}{l}\mathcal{I} \\
\dot{\forall}\end{array}$ & $\stackrel{\substack{ָ \\
\sim}}{ }$ & $\begin{array}{l}2 \\
\text { n }\end{array}$ & $\stackrel{g}{\circ}$ & $\begin{array}{c}\infty \\
m \\
m\end{array} \mid$ & $\begin{array}{l}\infty \\
\infty \\
i\end{array}$ & $\begin{array}{l}F \\
i\end{array}$ & 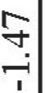 & $\begin{array}{l}\stackrel{2}{1} \\
\text { r. }\end{array}$ & $\begin{array}{l}0 \\
+ \\
+ \\
i\end{array}$ & $\begin{array}{l}+ \\
-\infty \\
-1\end{array}$ & $\begin{array}{c}n \\
i \\
i \\
i\end{array} \mid$ & $\begin{array}{l}2 \\
\vdots \\
0\end{array}$ & $\begin{array}{l}\mathfrak{i} \\
i\end{array}$ & 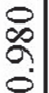 \\
\hline & 0 & & సู. & - & 尚: & $\bar{n}$ & $\frac{2}{\infty}$ & n̊. & 于 & $?$ & 文 & ले & $\underset{8}{8}$ & I & 1 & 1 & 1 & $\mathrm{I}$ \\
\hline \multirow{2}{*}{$\frac{N}{\frac{N}{0}}$} & $\theta^{\circ}$ & 극 & $\begin{array}{l}n \\
\stackrel{7}{+} \\
\stackrel{1}{*}\end{array}$ & $\begin{array}{l}1 \\
0 \\
0 \\
\end{array}$ & $\stackrel{\vartheta}{\circ}$ & $\stackrel{\circ}{\circ}$ & ñ & $\begin{array}{l}\overrightarrow{1} \\
\mathrm{i}\end{array}$ & $\frac{n}{-1}$ & \begin{tabular}{|c|}
8 \\
\\
\end{tabular} & \begin{tabular}{l} 
임 \\
\hdashline
\end{tabular} & $\begin{array}{c}9 \\
\text { m} \\
T\end{array}$ & \begin{tabular}{l}
\multirow{U}{*}{} \\
$\dot{\varphi}$ \\
$\dot{1}$
\end{tabular} & $\begin{array}{c}\text { A } \\
\text { in }\end{array}$ & $\begin{array}{l}\infty \\
2 \\
ن \\
ن\end{array}$ & $\stackrel{2}{-}$ & $\begin{array}{l}\text { D } \\
\text { in }\end{array}$ & $\begin{array}{l}8 \\
\vdots \\
0 \\
0\end{array}$ \\
\hline & $\stackrel{0}{0}$ & & $\frac{0}{6}$ & $\begin{array}{c}n \\
m \\
\infty\end{array}$ & ๙ેे & 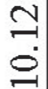 & $\begin{array}{l}\infty \\
\vdots \\
a\end{array}$ & $\begin{array}{l}0 \\
\stackrel{-}{0} \\
-\end{array}$ & $\begin{array}{l}\stackrel{2}{\infty} \\
\infty\end{array}$ & $=$ & $\begin{array}{l}n \\
n \\
0\end{array}$ & $\begin{array}{l}\text { in } \\
\text { in }\end{array}$ & $\underset{+}{\stackrel{f}{+}}$ & I & 1 & 1 & 1 & 1 \\
\hline \multirow{3}{*}{$\frac{7}{\frac{D}{5}}$} & $\delta^{\circ}$ & & 6 & రి. & $\stackrel{ }{!}$ & $\vec{\sim}$ & ले & ?̊n & $\stackrel{\circ}{\circ}$ & \& & $\begin{array}{l}m \\
0 \\
0\end{array}$ & f & กุ & $\stackrel{\infty}{2}$ & ?.? & $\stackrel{0}{0}$ & 2 & ลิ \\
\hline & & & & & & & & & & & & & & & & & & \\
\hline & 0 & & in & $\infty$ & a) & $\begin{array}{l}\infty \\
\sigma\end{array}$ & $\vec{a}$ & $\begin{array}{l}\infty \\
\infty \\
\alpha\end{array} \mid$ & $\infty$ & 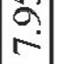 & $\begin{array}{l}n \\
6 \\
6\end{array}$ & in & $\underset{\sim}{*}$ & I & 1 & I & 1 & I \\
\hline \multicolumn{2}{|c|}{$(9 \mathrm{I} 0 Z-\angle 86 \mathrm{I}) m G$} & & $\begin{array}{l}\bar{\sigma} \\
\text { in }\end{array}$ & $\begin{array}{c}\mathscr{m} \\
\infty \\
\infty\end{array}$ & $\stackrel{m}{\tilde{m}}$ & $\stackrel{\sim}{\mathcal{O}}$ & $\stackrel{9}{8}$ & $\begin{array}{l}\infty \\
\infty \\
\infty\end{array}$ & $\underset{\infty}{\mathbb{N}}$ & $\begin{array}{l}\infty \\
9 \\
r\end{array}$ & 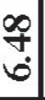 & $\begin{array}{l}N \\
N \\
\text { vi }\end{array}$ & $\begin{array}{l}0 \\
+ \\
+\end{array}$ & & & & & \\
\hline \multicolumn{2}{|c|}{ чрUоN } & & 8 & $\sum^{\bar{\pi}}$ & 荌 & $\stackrel{5}{\pi}$ & $\Xi$ & $\Xi$ & $\stackrel{\infty}{\nexists}$ & 今) & $\overline{0}$ & 官 & $\stackrel{8}{\mathscr{N}}$ & $\begin{array}{l}1 \\
2 \\
3\end{array}$ & $\mid \begin{array}{l}1 \\
\text { ara } \\
\frac{3}{4}\end{array}$ & s) & 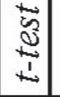 & 路 \\
\hline
\end{tabular}




\begin{tabular}{|c|c|c|c|c|c|c|c|c|c|c|c|c|c|c|c|c|c|c|}
\hline \multirow{2}{*}{$\begin{array}{l} \pm \\
\frac{\Xi}{\delta} \\
\frac{0}{2}\end{array}$} & $\stackrel{\circ}{\circ}$ & $\left|\begin{array}{c}\infty \\
\stackrel{\sim}{\sim}\end{array}\right|$ & $\begin{array}{l}2 \\
\infty \\
0 \\
1\end{array}$ & $\begin{array}{l}J \\
\stackrel{i}{*}\end{array}$ & $\begin{array}{l}\stackrel{0}{n} \\
\text { cri }\end{array}$ & \begin{tabular}{c}
$\stackrel{్}{\circ}$ \\
\hdashline
\end{tabular} & $\begin{array}{l}n \\
m \\
m\end{array}$ & $\begin{array}{c}\stackrel{f}{+} \\
\dot{r}\end{array}$ & 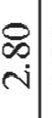 & $\stackrel{8}{-}$ & \begin{tabular}{l}
8 \\
\hdashline \\
-1
\end{tabular} & م̂م. & $=$ & $\stackrel{\overrightarrow{0}}{\mathrm{i}}$ & $\mid \begin{array}{c}0 \\
\infty \\
\grave{i} \\
\end{array}$ & $\stackrel{8}{8}$ & $\begin{array}{l}\infty \\
0 \\
\stackrel{j}{v}\end{array}$ & $\begin{array}{l}3 \\
\delta \\
\delta \\
\vdots\end{array}$ \\
\hline & $\stackrel{0}{0}$ & $\stackrel{9}{\partial}$ & $\begin{array}{l}2 \\
i\end{array}$ & $\stackrel{2}{\infty}$ & $\begin{array}{l}8 \\
8 \\
0\end{array}$ & $\begin{array}{l}8 \\
0 \\
0 \\
0\end{array}$ & $\bar{a}$ & 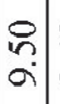 & $\begin{array}{c}\infty \\
+ \\
\infty\end{array}$ & $\begin{array}{l}\stackrel{n}{\infty} \\
\stackrel{\sim}{n}\end{array}$ & 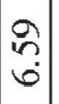 & $\begin{array}{l}\stackrel{2}{3} \\
n\end{array}$ & $\stackrel{m}{\tilde{r}}$ & I & 1 & $\mathrm{I}$ & 1 & I \\
\hline \multirow{2}{*}{$\frac{n}{\frac{0}{0}}$} & $\stackrel{\circ}{\circ}$ & 음 & $\begin{array}{l}\infty \\
0 \\
0 \\
0\end{array}$ & $\begin{array}{l}\triangleright \\
\$ \\
0\end{array}$ & 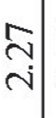 & $\begin{array}{l}? \\
\\
\end{array}$ & $\begin{array}{l}\overrightarrow{0} \\
i\end{array}$ & $\begin{array}{l}\vec{\sim} \\
\vec{i}\end{array}$ & $\stackrel{2}{2}$ & $\stackrel{p}{\stackrel{9}{0}}$ & ஸి & 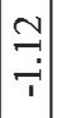 & $\stackrel{\sim}{\underset{-}{-}}$ & 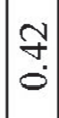 & $\stackrel{m}{0}$ & م્م & $\begin{array}{l}\stackrel{2}{\circ} \\
0\end{array}$ & 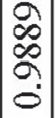 \\
\hline & $\stackrel{0}{0}$ & $\stackrel{\leftrightarrow}{+}$ & $\begin{array}{l}\mathfrak{\alpha} \\
n \\
n\end{array}$ & $\begin{array}{c}\infty \\
\infty \\
\infty\end{array}$ & $\stackrel{\sim}{\sim}$ & $\begin{array}{l}9 \\
\stackrel{9}{0}\end{array}$ & 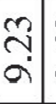 & 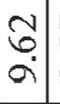 & $\begin{array}{l}n \\
\infty\end{array}$ & $\stackrel{2}{\swarrow}$ & $\begin{array}{c}3 \\
6 \\
0\end{array}$ & $\begin{array}{c}\infty \\
N \\
n \\
n\end{array}$ & $\begin{array}{l}\stackrel{\rho}{q} \\
\dot{\forall}\end{array}$ & $\mathrm{I}$ & 1 & I & $\mathrm{I}$ & $\mathrm{I}$ \\
\hline \multirow{2}{*}{$\begin{array}{l}\text { I } \\
\frac{0}{0} \\
\frac{0}{2}\end{array}$} & $\stackrel{\circ}{\circ}$ & స్. & 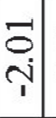 & $\begin{array}{l}\text { ㅎ. } \\
\text { గi. }\end{array}$ & $\infty$ & $\stackrel{\oplus}{-}$ & $\begin{array}{l}3 \\
0 \\
0\end{array}$ & 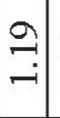 & $\begin{array}{l}2 \\
\infty \\
0 \\
0\end{array}$ & $\stackrel{5}{\mathrm{~N}}$ & 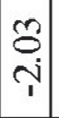 & 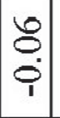 & $\begin{array}{l}\mathscr{2} \\
\stackrel{0}{0}\end{array}$ & $=$ & $\mid \begin{array}{l}0 \\
0 \\
\\
\end{array}$ & 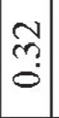 & $\frac{n}{0}$ & $\begin{array}{l}\stackrel{\circ}{\Omega} \\
\stackrel{2}{\circ}\end{array}$ \\
\hline & $\stackrel{0}{0}$ & ิㅜㅇ & $\begin{array}{l}\qquad \\
0 \\
0\end{array}$ & $\begin{array}{l}F \\
\infty\end{array}$ & 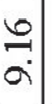 & $\begin{array}{l}\Omega \\
ૂ\end{array}$ & $\stackrel{n}{a}$ & $\vec{r}$ & $\begin{array}{l}6 \\
\infty \\
\infty\end{array}$ & $\frac{2}{2}$ & $\begin{array}{c}\overline{6} \\
\dot{0}\end{array}$ & \begin{tabular}{c}
\multirow{N}{N}{} \\
in
\end{tabular} & $\begin{array}{c}\stackrel{\mathcal{J}}{+} \\
\dot{f}\end{array}$ & $\mathrm{I}$ & 1 & $\mathrm{I}$ & $\mathrm{I}$ & 1 \\
\hline \multirow{2}{*}{$\begin{array}{l}= \\
\text { 일 } \\
\frac{0}{2}\end{array}$} & $\dot{\theta}^{0}$ & $\stackrel{N}{-}$ & $\begin{array}{l}\dddot{2} \\
\hat{\varphi}\end{array}$ & 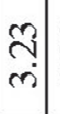 & 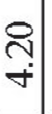 & 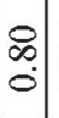 & $\begin{array}{l}2 \\
\dot{m}\end{array}$ & $\begin{array}{l}n \\
0 \\
f\end{array}$ & $\begin{array}{c}N \\
m \\
m\end{array}$ & $\underset{⿱ 亠}{\infty}$ & 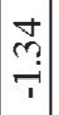 & 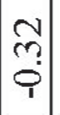 & $\begin{array}{l}\dot{O} \\
\stackrel{i}{ }\end{array}$ & $\begin{array}{l}\stackrel{B}{6} \\
\stackrel{i}{ }\end{array}$ & $\begin{array}{l}2 \\
0 \\
0\end{array}$ & $\mid \begin{array}{c}\infty \\
\infty \\
0\end{array}$ & $=$ & 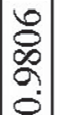 \\
\hline & $\stackrel{\Delta}{0}$ & 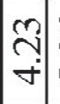 & $\begin{array}{l}\circ \\
\searrow \\
n\end{array}$ & $\begin{array}{c}\infty \\
0 \\
\infty\end{array}$ & $\begin{array}{l}+ \\
\infty \\
\infty\end{array}$ & $\begin{array}{l} \pm \\
0 \\
0 \\
0\end{array}$ & $\begin{array}{l}\qquad \\
0 \\
0\end{array}$ & $\begin{array}{c}\stackrel{?}{f} \\
\sigma\end{array}$ & $\begin{array}{c}m \\
\infty \\
\infty\end{array}$ & $\begin{array}{l}\infty \\
\stackrel{\infty}{N}\end{array}$ & ñ. & \begin{tabular}{c}
\multirow{N}{N}{} \\
$i$
\end{tabular} & $\stackrel{m}{\sim}$ & $\mathrm{I}$ & 1 & I & I & I \\
\hline \multicolumn{2}{|c|}{$(9 \mathrm{I} 0 \mathrm{z}-\angle 86 \mathrm{I}) u \sigma$} & & & $\begin{array}{c}10 \\
\infty \\
\infty\end{array}$ & $\stackrel{\tilde{\sigma}}{a}$ & $\stackrel{\mathcal{N}}{\stackrel{\mathcal{\Theta}}{\Theta}}$ & $\stackrel{p}{a}$ & $\begin{array}{l}\infty \\
\infty \\
\sigma\end{array}$ & $\underset{\infty}{\mathbb{N}}$ & $\begin{array}{l}\infty \\
\stackrel{5}{r}\end{array}$ & 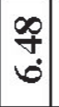 & 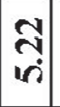 & $\begin{array}{l}0 \\
\text { ơ } \\
+\end{array}$ & & & & & \\
\hline \multicolumn{2}{|c|}{ ч1чоб } & : & $\begin{array}{c}0 \\
0 \\
0 \\
0\end{array}$ & 弯 & 豪 & 空 & $\Xi$ & $\Xi$ & 尝 & : & $\overrightarrow{0}$ & $\begin{array}{l}z \\
z \\
z\end{array}$ & $\begin{array}{l}8 \\
0 \\
0\end{array}$ & $\begin{array}{l}101 \\
3 \\
3\end{array}$ & $\mid \begin{array}{l}1 \\
00 \\
3 \\
z\end{array}$ & $\frac{1}{3}$ & & $\begin{array}{l}\text { 列 } \\
5 \\
2 \\
2\end{array}$ \\
\hline
\end{tabular}




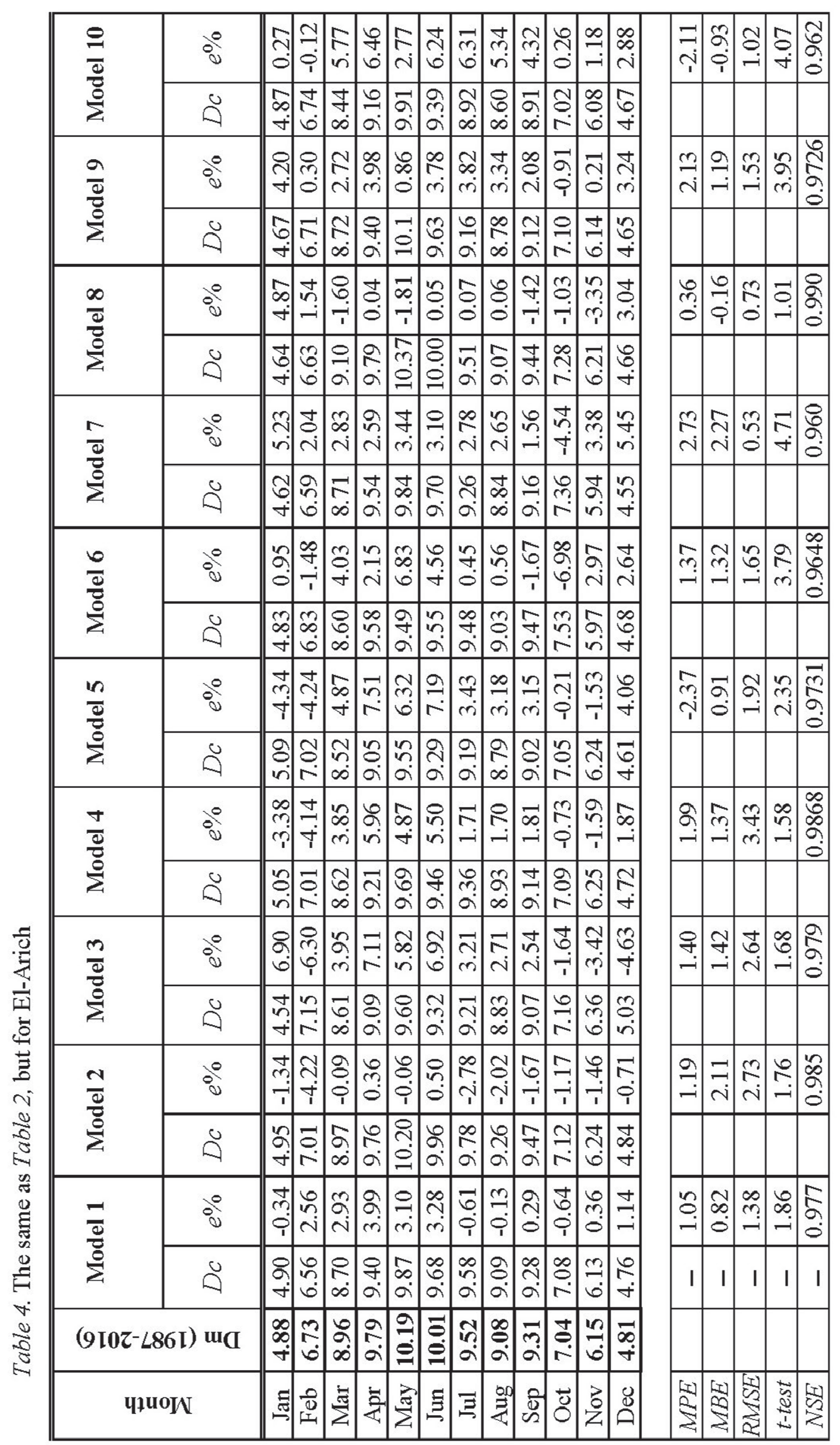




\begin{tabular}{|c|c|c|c|c|c|c|c|c|c|c|c|c|c|c|c|c|c|c|}
\hline \multirow{2}{*}{ 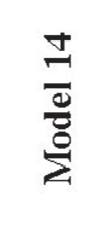 } & $\dot{o}_{0}^{\circ}$ & $\begin{array}{l}\infty \\
0 \\
0\end{array}$ & $\begin{array}{l}\underset{6}{1} \\
\stackrel{i}{c}\end{array}$ & $\mid \begin{array}{c}\vec{J} \\
\dot{v}\end{array}$ & 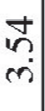 & 고 & $\begin{array}{l}m \\
m \\
m\end{array}$ & 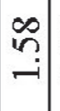 & $\begin{array}{c}\infty \\
m \\
m \\
m\end{array}$ & $\underset{\mathrm{i}}{\mathrm{N}}$ & $\begin{array}{l}\infty \\
0 \\
-i \\
\end{array}$ & $\begin{array}{l}\mathrm{g} \\
\mathrm{i}\end{array}$ & $\mid \begin{array}{l}\mathbf{6} \\
0 \\
0\end{array}$ & $\stackrel{m}{m}$ & \begin{tabular}{|l}
$\bar{\alpha}$ \\
$\hat{i}$ \\
1
\end{tabular} & $\begin{array}{l}+ \\
0 \\
0\end{array}$ & 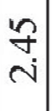 & $\frac{J}{2}$ \\
\hline & $\stackrel{0}{0}$ & $\stackrel{\curvearrowright}{\gamma}$ & $\begin{array}{l}\infty \\
\stackrel{n}{6} \\
\end{array}$ & $\mid \begin{array}{c} \pm \\
\infty \\
\infty\end{array}$ & 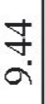 & $\begin{array}{l}0 \\
\stackrel{0}{0} \\
-\end{array}$ & 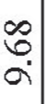 & $\begin{array}{l}n \\
2 \\
a\end{array}$ & $\stackrel{F}{\infty}$ & $\begin{array}{l}8 \\
0 \\
a\end{array}$ & $\begin{array}{c}0 \\
\frac{1}{2}\end{array}$ & $\left|\begin{array}{c}0 \\
0 \\
0\end{array}\right|$ & $\begin{array}{l}+ \\
+ \\
+\end{array}$ & $\mathrm{I}$ & 1 & 1 & 1 & 1 \\
\hline \multirow{2}{*}{$\frac{m}{\frac{m}{0}}$} & $\dot{o}^{\circ}$ & $\stackrel{\nabla}{9}$ & $\stackrel{5}{\circ}$ & $\begin{array}{c}+ \\
\infty \\
0 \\
\dot{0}\end{array}$ & הֶ̃ & $\frac{m}{9}$ & 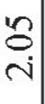 & $\begin{array}{l}7 \\
\\
\end{array}$ & $\begin{array}{l}\infty \\
\stackrel{0}{0} \\
\dot{\mathrm{j}}\end{array}$ & $\stackrel{?}{=}$ & $\begin{array}{c}\mathrm{T} \\
\mathrm{T}\end{array}$ & $\underset{-}{\stackrel{N}{-}}$ & $\mid$\begin{tabular}{l}
$n$ \\
\hdashline \\
\hdashline \\
1
\end{tabular} & $\vec{m}$ & 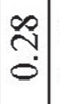 & $\begin{array}{l}\text { gे } \\
\dot{0}\end{array}$ & $\stackrel{8}{\circ}$ & $\stackrel{2}{\Omega}$ \\
\hline & $\stackrel{0}{0}$ & & $\frac{m}{6}$ & $\begin{array}{l}\infty \\
\infty \\
\infty \\
\infty\end{array} \mid$ & $\begin{array}{l}\tilde{n} \\
i\end{array}$ & 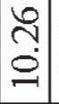 & $\begin{array}{l}8 \\
\infty \\
\infty\end{array}$ & \begin{tabular}{c}
$\infty$ \\
\multirow{\sigma}{*}{} \\
$\alpha$
\end{tabular} & $\begin{array}{c}\infty \\
\infty \\
\infty \\
\infty\end{array}$ & $\begin{array}{c}n \\
a \\
a\end{array}$ & 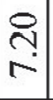 & $\begin{array}{l}\infty \\
0 \\
0 \\
0\end{array} \mid$ & $\begin{array}{l}\infty \\
\dot{+}\end{array}$ & $\mathrm{I}$ & 1 & 1 & $\mathrm{I}$ & 1 \\
\hline \multirow{2}{*}{$\frac{\sqrt{3}}{\frac{\sigma}{0}}$} & iे & 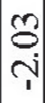 & $\stackrel{\mathbb{N}}{0}$ & 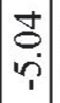 & $\underset{\infty}{\infty}$ & 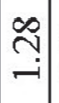 & $\begin{array}{l}\bar{b} \\
\dot{0}\end{array}$ & $\begin{array}{c}\infty \\
\infty \\
0\end{array}$ & î̀ & $\begin{array}{l}\text { ב } \\
= \\
-\end{array}$ & 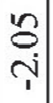 & $\stackrel{2}{\circ}$ & $\mid \begin{array}{l}0 \\
0 \\
0 \\
1 \\
1\end{array}$ & $\begin{array}{l}\infty \\
0 \\
0 \\
\dot{1}\end{array}$ & $\stackrel{m}{\overrightarrow{0}}$ & Nָ & $\frac{9}{0}$ & 2 \\
\hline & $\stackrel{0}{0}$ & 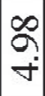 & $\frac{7}{6}$ & $\mid \begin{array}{c}\vec{y} \\
\dot{\sigma}\end{array}$ & \begin{tabular}{l}
$\vec{b}$ \\
\hdashline
\end{tabular} & $\mid \begin{array}{l}0 \\
\stackrel{1}{0} \\
0\end{array}$ & $\begin{array}{l}2 \\
\alpha\end{array}$ & \begin{tabular}{l}
$J$ \\
\multirow{2}{*}{}
\end{tabular} & $\begin{array}{l}\infty \\
\infty \\
\infty\end{array}$ & $\begin{array}{c}\stackrel{ }{a} \\
\text { | }\end{array}$ & $\begin{array}{l}\infty \\
r \\
r\end{array}$ & $\left|\begin{array}{c}8 \\
0 \\
0\end{array}\right|$ & $\begin{array}{l}-\vec{\infty} \\
\dot{\nabla}\end{array}$ & $\mathrm{I}$ & 1 & 1 & 1 & I \\
\hline \multirow{2}{*}{$\begin{array}{l}\bar{\Xi} \\
\bar{\Xi} \\
\sum\end{array}$} & $\stackrel{8}{\circ}$ & $\stackrel{\infty}{\stackrel{\infty}{\sim}}$ & $\stackrel{\sim}{\mathrm{O}}$ & $\begin{array}{l}\text { શે } \\
+\end{array}$ & 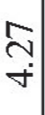 & $\begin{array}{l}\infty \\
\infty \\
-\end{array}$ & $\begin{array}{l}8 \\
\stackrel{8}{ } \\
\dot{n}\end{array}$ & $\begin{array}{l}= \\
\text { in }\end{array}$ & શે & $\begin{array}{c}\stackrel{n}{m} \\
m\end{array}$ & 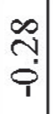 & $\begin{array}{l}J \\
0\end{array}$ & $\begin{array}{l}\exists \\
\dot{m}\end{array}$ & बे & $=$ & $\mid \begin{array}{c}\infty \\
\infty \\
-i\end{array}$ & $\begin{array}{c}\infty \\
i \\
i\end{array}$ & $\begin{array}{l}\stackrel{2}{2} \\
\stackrel{2}{\circ} \\
0\end{array}$ \\
\hline & ڤ & $\stackrel{F}{\stackrel{F}{+}}$ & $\overrightarrow{6}$ & $\begin{array}{c}\infty \\
i \\
\infty \\
\infty\end{array} \mid$ & $\stackrel{\tilde{m}}{\tilde{a}}$ & $\begin{array}{l}8 \\
\stackrel{0}{0}\end{array}$ & $\begin{array}{l}n \\
\vdots \\
\vdots\end{array}$ & $\begin{array}{l}3 \\
0 \\
0\end{array} \mid$ & 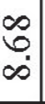 & $\begin{array}{l}\overline{0} \\
\dot{a}\end{array}$ & $\begin{array}{l}0 \\
8 \\
\end{array}$ & $\left|\begin{array}{c}0 \\
\hdashline \\
6\end{array}\right|$ & $\begin{array}{l}0 \\
\ddots \\
+ \\
\end{array}$ & I & 1 & 1 & $\mathrm{I}$ & I \\
\hline \multicolumn{2}{|c|}{$(9 \mathrm{I} 0 Z-\angle 86 \mathrm{I}) \mathrm{u}_{\mathrm{C}}$} & 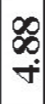 & $\frac{\pi}{6}$ & $\mid \begin{array}{c}2 \\
\infty \\
\infty\end{array}$ & $\begin{array}{l}2 \\
2 \\
2\end{array}$ & $\begin{array}{l}\frac{a}{\theta} \\
\theta\end{array}$ & $\begin{array}{l}\bar{\theta} \\
\stackrel{\theta}{\theta}\end{array}$ & 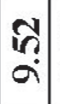 & 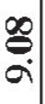 & $\bar{\sigma}$ & $\underset{r}{ \pm}$ & $\frac{1}{6}$ & $\begin{array}{l}\vec{\infty} \\
\dot{\tau}\end{array}$ & & & & & \\
\hline \multicolumn{2}{|c|}{ чұบоМ } & ت & 0 & $\sum_{i}^{\pi}$ & 荎 & $\sum_{\Sigma}^{\vec{m}}$ & $\Xi$ & $\Xi$ & $\stackrel{00}{\Xi}$ & ڤे & $\overrightarrow{0}$ & $\mid \begin{array}{l}z \\
0 \\
z\end{array}$ & $\begin{array}{l}8 \\
\mathscr{D}\end{array}$ & $\begin{array}{l}{\left[\begin{array}{ll}1 \\
0 \\
3\end{array}\right.} \\
\vdots\end{array}$ & $\begin{array}{l}\text { and } \\
\frac{2}{2}\end{array}$ & $\frac{n}{3}$ & 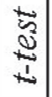 & $\begin{array}{l}\frac{1}{5} \\
\text { 曾 }\end{array}$ \\
\hline
\end{tabular}




\begin{tabular}{|c|c|c|c|c|c|c|c|c|c|c|c|c|c|c|c|c|c|c|}
\hline \multirow{2}{*}{$\begin{array}{l}\varrho \\
\frac{0}{g} \\
\frac{\sigma}{2}\end{array}$} & $o_{0}^{\circ}$ & ㄱ. & $\begin{array}{l}8 \\
\stackrel{0}{0} \\
\end{array}$ & $\begin{array}{c}2 \\
0 \\
i\end{array}$ & $\begin{array}{l}\text { ?口 } \\
\text { ñ. }\end{array}$ & $\bar{m}$ & $\begin{array}{l}1 \\
6 \\
r\end{array}$ & $\begin{array}{l}F \\
\text { in }\end{array}$ & $\begin{array}{l}0 \\
\infty \\
\forall \\
\forall\end{array}$ & $\begin{array}{c}\infty \\
\sim \\
\dot{r}\end{array} \mid$ & 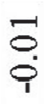 & $\begin{array}{l}\wp \\
\circ\end{array}$ & $\begin{array}{l}\text { ஓे } \\
\text { ㄱ. }\end{array}$ & $\begin{array}{c}0 \\
-1 \\
-1\end{array}$ & $\mid \begin{array}{c}0 \\
+ \\
\dot{1} \\
1\end{array}$ & $\left|\begin{array}{l}\mathcal{W} \\
\dot{0}\end{array}\right|$ & & $\mid \begin{array}{c}\infty \\
\infty \\
0 \\
0\end{array}$ \\
\hline & $\stackrel{\Delta}{\Delta}$ & 9 & $\begin{array}{l}8 \\
6 \\
\end{array}$ & $\begin{array}{c}2 \\
\infty \\
\infty\end{array}$ & $\begin{array}{l}\infty \\
i \\
i\end{array}$ & \begin{tabular}{c|} 
\\
$\dot{\gamma}$ \\
\end{tabular} & $\begin{array}{l}n \\
\infty \\
\infty\end{array}$ & 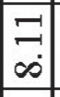 & $\begin{array}{l} \pm \\
0 \\
\end{array}$ & $\begin{array}{l}0 \\
\infty \\
-\end{array}$ & 守 & $\begin{array}{c}0 \\
\text { in } \\
\end{array}$ & $\begin{array}{l}\vec{y} \\
\dot{\sigma}\end{array}$ & 1 & 1 & 1 & 1 & $\mathrm{I}$ \\
\hline \multirow{2}{*}{$\frac{a}{\frac{0}{0}}$} & 8 & 6 & $\stackrel{\overbrace{}}{\sim}$ & 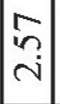 & $\begin{array}{l}\stackrel{0}{r} \\
m\end{array}$ & $\left(\begin{array}{r}n \\
0\end{array}\right.$ & $\begin{array}{l}n \\
n \\
m\end{array}$ & 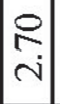 & $\begin{array}{l}0 \\
m \\
m\end{array}$ & $\stackrel{\stackrel{m}{+}}{\stackrel{\sim}{\sim}}$ & $\stackrel{\overbrace{}}{?}$ & 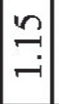 & $\stackrel{m}{-1}$ & $\begin{array}{l}0 \\
\stackrel{0}{n} \\
i\end{array}$ & $\stackrel{n}{\beth}$ & $\mid \begin{array}{c}2 \\
\stackrel{2}{2}\end{array}$ & 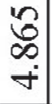 & $\left|\begin{array}{l}\bar{b} \\
0 \\
0 \\
0\end{array}\right|$ \\
\hline & ڤั & & $\begin{array}{l}\Omega \\
\text { in }\end{array}$ & $\mid \begin{array}{l}8 \\
\infty \\
\infty\end{array}$ & $\begin{array}{l}\dot{m} \\
\vdots \\
\vdots\end{array}$ & $\begin{array}{c}3 \\
0 \\
2\end{array}$ & $\begin{array}{l}P \\
\infty \\
\infty\end{array}$ & $\begin{array}{c}\infty \\
\infty \\
\infty\end{array}$ & $\begin{array}{c}5 \\
\infty \\
\infty\end{array}$ & $\begin{array}{l}2 \\
-1 \\
\end{array}$ & ñ & 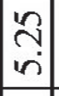 & $\begin{array}{l}\text { qे } \\
+\end{array}$ & 1 & 1 & 1 & $\mathrm{I}$ & 1 \\
\hline \multirow{2}{*}{$\frac{\infty}{0}$} & oे & \begin{tabular}{l}
$\infty$ \\
$\infty$ \\
\hdashline \\
1
\end{tabular} & $\exists$ & $\left(\begin{array}{c}\infty \\
\stackrel{-1}{-}\end{array}\right.$ & $\begin{array}{l}\stackrel{2}{\infty} \\
\text { ì }\end{array}$ & $\begin{array}{l}3 \\
\hat{1} \\
\hat{i}\end{array}$ & $\begin{array}{l}\hat{b} \\
\text { in }\end{array}$ & $\begin{array}{l}2 \\
\hat{1} \\
\end{array}$ & $\begin{array}{l}m \\
i \\
i\end{array}$ & กั & 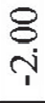 & $\begin{array}{c}0 \\
-6 \\
-1\end{array}$ & 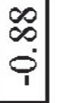 & 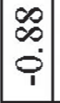 & 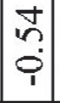 & $\mid \begin{array}{l}\infty \\
\infty \\
0 \\
0\end{array}$ & ñ & $\begin{array}{l}\infty \\
\curvearrowright \\
\delta \\
0\end{array}$ \\
\hline & $\stackrel{0}{0}$ & & $\begin{array}{l}\Omega \\
\text { in }\end{array}$ & $\left|\begin{array}{c}N \\
N \\
\infty\end{array}\right|$ & 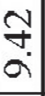 & $\begin{array}{l}7 \\
2\end{array}$ & $\begin{array}{l}\infty \\
2 \\
\infty\end{array}$ & $\begin{array}{c}2 \\
6 \\
\infty \\
\infty\end{array}$ & $\frac{\mathcal{N}}{\infty}$ & $\frac{2}{2}$ & $\begin{array}{l}8 \\
\stackrel{8}{0}\end{array}$ & \begin{tabular}{c}
\multirow{2}{N}{} \\
in
\end{tabular} & $\begin{array}{l}0 \\
\stackrel{5}{+}\end{array}$ & 1 & 1 & I & 1 & $\mathrm{I}$ \\
\hline \multirow{2}{*}{$\frac{\pi}{\frac{\sigma}{0}}$} & $\stackrel{0}{0}$ & $\begin{array}{l}n \\
n \\
n\end{array}$ & $\frac{2}{2}$ & $\left|\begin{array}{c}\tilde{W} \\
\dot{\circ}\end{array}\right|$ & $\vec{?}$ & $\mid \begin{array}{l}\infty \\
\dot{0}\end{array}$ & $\stackrel{\infty}{\stackrel{n}{-}}$ & $\stackrel{?}{\stackrel{P}{-}}$ & $\begin{array}{c}0 \\
-i \\
-1\end{array}$ & $\mid \begin{array}{l}5 \\
\circ \\
0\end{array}$ & 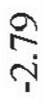 & $\mid \begin{array}{l}\stackrel{0}{0} \\
\circ\end{array}$ & $\underset{\sim}{\stackrel{\sim}{+}}$ & $\left|\begin{array}{c}\stackrel{m}{\sim} \\
\stackrel{\sim}{*}\end{array}\right|$ & $\stackrel{m}{-2}$ & 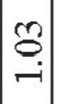 & $\stackrel{m}{\stackrel{\leftrightarrow}{+}}$ & $\begin{array}{l}1 \\
8 \\
8 \\
0\end{array}$ \\
\hline & $\stackrel{0}{a}$ & & 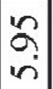 & $\left|\begin{array}{c}\infty \\
+\infty \\
\infty\end{array}\right|$ & $\hat{n}$ & $\begin{array}{l}\vec{b} \\
\dot{a}\end{array}$ & $\begin{array}{l}\infty \\
\infty \\
\infty\end{array} \mid$ & $\begin{array}{c}\infty \\
+ \\
\infty\end{array} \mid$ & \begin{tabular}{c}
\multirow{N}{N}{} \\
$\infty$
\end{tabular} & $\left|\begin{array}{c}N \\
-\infty\end{array}\right|$ & : & m. & $\begin{array}{l}0 \\
\stackrel{\Upsilon}{+}\end{array}$ & 1 & 1 & 1 & $\mathrm{I}$ & $\mathrm{I}$ \\
\hline \multirow{2}{*}{$\begin{array}{l}0 \\
\frac{0}{0} \\
\frac{0}{2}\end{array}$} & & वे. & 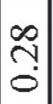 & $\left|\begin{array}{c}\stackrel{9}{+} \\
\dot{r}\end{array}\right|$ & $\stackrel{\overbrace{}}{\overparen{3}}$ & $\frac{m}{m}$ & $\begin{array}{l}\infty \\
\infty \\
m\end{array}$ & $\mid \begin{array}{c}\mathcal{\sigma} \\
- \\
-\end{array}$ & $\stackrel{-0}{-}$ & $\mid \begin{array}{l}8 \\
\circ \\
\dot{0} \\
1\end{array}$ & $\begin{array}{l}\text { in } \\
\text { in }\end{array}$ & $\left|\begin{array}{c}\infty \\
\stackrel{1}{m}\end{array}\right|$ & $\begin{array}{l}\stackrel{2}{ } \\
\dot{\gamma}\end{array}$ & $\left|\begin{array}{c}1 \\
\infty \\
\infty \\
0\end{array}\right|$ & $\mid \begin{array}{c}\infty \\
n \\
0 \\
0\end{array}$ & $\begin{array}{l}0 \\
- \\
-1\end{array}$ & & $\begin{array}{l}2 \\
2 \\
2 \\
2\end{array}$ \\
\hline & $\ddot{0}$ & 守 & $\begin{array}{l}\overrightarrow{0} \\
0 \\
\end{array}$ & $\begin{array}{c}m \\
n \\
\infty\end{array}$ & 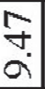 & $\begin{array}{c}a \\
-2\end{array}$ & $\begin{array}{l}6 \\
\infty \\
\infty\end{array}$ & $\mid \begin{array}{c}0 \\
+ \\
\infty \\
\infty\end{array}$ & $\begin{array}{l}N \\
\text { Na } \\
\infty\end{array}$ & \begin{tabular}{c|}
$m$ \\
$\infty$ \\
$\infty$
\end{tabular} & $\begin{array}{l}\text { षे } \\
\text { ठ }\end{array}$ & $\begin{array}{l} \pm \\
\text { in }\end{array}$ & $\stackrel{n}{\stackrel{9}{*}}$ & 1 & 1 & 1 & 1 & $\mathrm{I}$ \\
\hline \multirow{2}{*}{$\frac{n}{2}$} & & - & $\begin{array}{l}0 \\
\infty \\
ن \\
ن\end{array}$ & $\begin{array}{l}\stackrel{9}{J} \\
\dot{\nabla}\end{array}$ & $\begin{array}{l}\hat{\infty} \\
\dot{+}\end{array}$ & $\left(\begin{array}{c}n \\
6\end{array}\right.$ & $\begin{array}{l}\infty \\
\infty \\
\text { in }\end{array}$ & $\stackrel{\Xi}{-}$ & $\stackrel{\infty}{-}$ & $\begin{array}{l} \pm \\
0\end{array}$ & $\begin{array}{l}8 \\
\ddot{1}\end{array}$ & $\begin{array}{c}N \\
0\end{array}$ & $m$ & $\stackrel{\infty}{\stackrel{\infty}{0}}$ & $\stackrel{\substack{n \\
-}}{-1}$ & $\stackrel{3}{-}$ & & $\mid \begin{array}{l}5 \\
8 \\
8 \\
0\end{array}$ \\
\hline & $\stackrel{0}{0}$ & & $\begin{array}{c}\hat{3} \\
6\end{array}$ & $\left|\begin{array}{c}7 \\
0 \\
\infty\end{array}\right|$ & 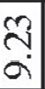 & $\left|\begin{array}{l}0 \\
0 \\
a\end{array}\right|$ & \begin{tabular}{c}
0 \\
\multirow{+}{\infty}{} \\
$\infty$
\end{tabular} & $\left|\begin{array}{c}\tilde{\gamma} \\
\infty\end{array}\right|$ & $\frac{9}{\infty}$ & $\left|\begin{array}{c}0 \\
\infty \\
\infty\end{array}\right|$ & $\frac{8}{6}$ & $\begin{array}{l}\text { N. } \\
\text { in }\end{array}$ & $\stackrel{\text { 여 }}{+}$ & 1 & $\mathrm{I}$ & 1 & 1 & $\mathrm{I}$ \\
\hline \multirow{2}{*}{$\frac{ \pm}{\frac{J}{0}}$} & & רั? & $\begin{array}{c}m \\
\ddot{r} \\
\dot{1}\end{array}$ & $\left|\begin{array}{l}+ \\
\infty \\
\dot{N}\end{array}\right|$ & $\begin{array}{l}8 \\
0 \\
0\end{array}$ & $\begin{array}{c}\stackrel{\odot}{\infty} \\
\dot{+}\end{array} \mid$ & $\begin{array}{l}\stackrel{2}{\mathrm{r}} \\
\dot{+}\end{array}$ & 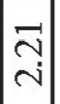 & $\begin{array}{l}\stackrel{R}{r} \\
\dot{m}\end{array}$ & $\begin{array}{l}8 \\
\dot{+}\end{array}$ & ळ̊ & $\begin{array}{c}\infty \\
\sim \\
i \\
i\end{array}$ & $\begin{array}{l}\mathscr{2} \\
\text { m. }\end{array}$ & $\mid$\begin{tabular}{l}
$\infty$ \\
\hdashline \\
-
\end{tabular} & m. & 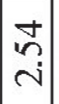 & & $\mid \begin{array}{l}\infty \\
2 \\
\vdots \\
0 \\
0\end{array}$ \\
\hline & $\stackrel{0}{0}$ & ेे & $\begin{array}{l}2 \\
6 \\
6\end{array}$ & $\left|\begin{array}{c}\infty \\
n \\
\infty\end{array}\right|$ & $\vec{z}=$ & 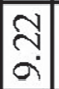 & $\begin{array}{c}\mathbb{J} \\
\infty \\
\infty\end{array} \mid$ & $\mid \begin{array}{c}\vec{y} \\
\infty\end{array}$ & 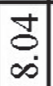 & $\begin{array}{c}\infty \\
\infty \\
-1\end{array}$ & గn & $\begin{array}{c}0 \\
\dot{n} \\
\dot{1}\end{array}$ & $\stackrel{m}{+}$ & 1 & 1 & 1 & 1 & 1 \\
\hline \multirow{2}{*}{$\frac{m}{\tilde{c}}$} & of & & $\begin{array}{l}m \\
m \\
r\end{array}$ & $\begin{array}{c}n \\
2 \\
m\end{array} \mid$ & $\underset{-}{ت}$ & $\begin{array}{c}\infty \\
\infty \\
i\end{array}$ & ఫิ & $\mid \begin{array}{c}\vec{N} \\
m\end{array}$ & $\vec{i}$ & 芯 & $\frac{\text { to }}{-}$ & $\begin{array}{c}\mathcal{T} \\
\dot{r} \\
i\end{array}$ & $\frac{9}{r}$ & $\begin{array}{l}1 \\
\dot{0} \\
\dot{1}\end{array}$ & $\left(\begin{array}{l}0 \\
-1 \\
-1\end{array}\right.$ & $\mid \begin{array}{c}\infty \\
\stackrel{\sim}{v} \\
\text { | }\end{array}$ & & $\left|\begin{array}{l}0 \\
\vdots \\
0 \\
0\end{array}\right|$ \\
\hline & 0 & 움 & 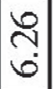 & $\left|\begin{array}{c}\infty \\
+ \\
\infty\end{array}\right|$ & $\begin{array}{l}\overrightarrow{0} \\
\vdots \\
\vdots\end{array}$ & $\frac{m}{a}$ & $\begin{array}{c}f \\
\infty \\
\infty\end{array}$ & $\left|\begin{array}{c}1 \\
m \\
\infty\end{array}\right|$ & $\begin{array}{c}\frac{1}{\infty} \\
\infty\end{array}$ & $\frac{\Omega}{\sim}$ & in & $\begin{array}{c}9 \\
\text { in. }\end{array}$ & $\begin{array}{l}8 \\
\dot{+}\end{array}$ & 1 & 1 & 1 & $\mathrm{I}$ & 1 \\
\hline \multirow{2}{*}{$\frac{N}{\frac{N}{g}}$} & $\delta_{0}^{\circ}$ & & $\begin{array}{c}m \\
n \\
r \\
r\end{array}$ & $\mid \begin{array}{l}0 \\
0 \\
0\end{array}$ & ָָ & $\begin{array}{l}2 \\
0\end{array}$ & $\stackrel{0}{-}$ & 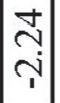 & 资 & $\begin{array}{l}\infty \\
= \\
T\end{array} \mid$ & $\frac{d}{T}$ & $\mid \begin{array}{c}- \\
-1 \\
\end{array}$ & 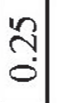 & 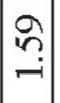 & $\stackrel{ \pm}{I}$ & $\begin{array}{c}\infty \\
0 \\
\dot{m}\end{array}$ & & $\mid \begin{array}{l}0 \\
\stackrel{2}{0} \\
\stackrel{0}{0}\end{array}$ \\
\hline & 8 & $\begin{array}{l}\text { बे } \\
\dot{\nabla}\end{array}$ & 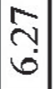 & $\left|\begin{array}{c}2 \\
\infty \\
\infty\end{array}\right|$ & \begin{tabular}{l}
$\infty$ \\
$\infty$ \\
\hdashline
\end{tabular} & 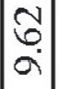 & $\begin{array}{c}\bar{\sigma} \\
\infty\end{array}$ & $\left|\begin{array}{c}2 \\
8 \\
\infty\end{array}\right|$ & $\begin{array}{c}\infty \\
+ \\
\infty\end{array}$ & {$\left[\begin{array}{c}2 \\
\infty \\
\infty\end{array}\right]$} & "n & $\left.\begin{array}{c}0 \\
\\
i\end{array}\right]$ & $\stackrel{\nabla}{\sim}$ & 1 & 1 & 1 & 1 & 1 \\
\hline \multirow{2}{*}{$\frac{\bar{c}}{\frac{\sigma}{0}}$} & 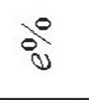 & & $\begin{array}{c}\mathscr{\infty} \\
\dot{0}\end{array}$ & $\stackrel{\mathcal{Y}}{\stackrel{\mathcal{V}}{-i}}$ & $\overrightarrow{-}$ & 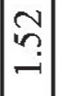 & $\stackrel{0}{\infty}$ & $\begin{array}{l}\Omega \\
- \\
-1\end{array}$ & S. & $\begin{array}{l}9 \\
0 \\
0\end{array} \mid$ & ஜ & 垈 & $\stackrel{-1}{\stackrel{c}{0}}$ & 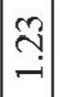 & $\stackrel{\sim}{\sim}$ & $\left|\begin{array}{c}\overrightarrow{0} \\
\stackrel{\sim}{i}\end{array}\right|$ & & $\begin{array}{c}\infty \\
\vdots \\
0 \\
0\end{array}$ \\
\hline & $\stackrel{0}{0}$ & & $\begin{array}{l}1 \\
0 \\
0\end{array}$ & $\left|\begin{array}{c}R \\
\infty\end{array}\right|$ & $\begin{array}{l}\text { a } \\
\text { aे }\end{array}$ & 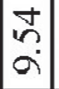 & $\begin{array}{l}\infty \\
\infty \\
\infty\end{array}$ & 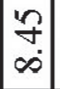 & $\begin{array}{c}\stackrel{0}{1} \\
\infty\end{array}$ & $\left|\begin{array}{c}5 \\
\infty \\
\infty\end{array}\right|$ & ت্ & $\left.\begin{array}{c}\infty \\
i \\
i\end{array}\right]$ & $\begin{array}{l}\stackrel{+}{\sim} \\
\dot{*}\end{array}$ & 1 & 1 & $\mathrm{I}$ & $\mathrm{I}$ & 1 \\
\hline \multicolumn{2}{|c|}{$(9 I 0 z-\angle 86 I) u_{a} G$} & $\begin{array}{l}9 \\
7 \\
\dot{0}\end{array}$ & $\begin{array}{l}0 \\
6 \\
0 \\
0\end{array}$ & $\left|\begin{array}{l}\infty \\
\infty \\
\infty\end{array}\right|$ & $\begin{array}{l}\stackrel{?}{2} \\
\dot{2}\end{array}$ & $\left|\begin{array}{c}\dot{a} \\
\hat{\alpha}\end{array}\right|$ & 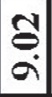 & $\mid \begin{array}{c}\overline{0} \\
\infty \\
\infty\end{array}$ & $\begin{array}{c}n \\
\infty \\
\infty\end{array}$ & $\frac{m}{\infty}$ & 于े & $\begin{array}{l}\bar{m} \\
\dot{n}\end{array}$ & in & & & & & \\
\hline \multicolumn{2}{|c|}{ чңшоО } & 娪 & & $\bar{z}$ & 菅 & 密 & $\Xi$ & $\Xi$ & $\stackrel{80}{\Xi}$ & 용 & $\overline{0}$ & $\begin{array}{l}3 \\
0 \\
2\end{array}$ & $\stackrel{\mathscr{U}}{0}$ & $\begin{array}{l}1 \\
0 \\
3 \\
\vdots\end{array}$ & 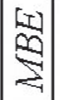 & $\frac{5}{\Omega}$ & 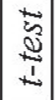 & $\begin{array}{l}\text { 窇 } \\
Z \\
Z\end{array}$ \\
\hline
\end{tabular}




\begin{tabular}{|c|c|c|c|c|c|c|c|c|c|c|c|c|c|c|c|c|c|c|}
\hline \multirow{2}{*}{ 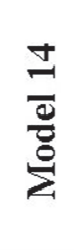 } & $i_{0}^{0}$ & $\infty$ & $\stackrel{+}{?}$ & 守 & $\stackrel{F}{i}$ & $\begin{array}{l}0 \\
0 \\
0 \\
1\end{array}$ & $\begin{array}{l}\tilde{\sigma} \\
\stackrel{\sim}{*}\end{array}$ & $\stackrel{?}{-}$ & $\stackrel{+}{\sim}$ & nn & ma & 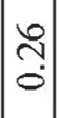 & 구 & {$\left[\begin{array}{c}n \\
0\end{array}\right.$} & $\begin{array}{l}F \\
\dot{0}\end{array}$ & $\begin{array}{l} \pm \\
0 \\
0\end{array}$ & $\stackrel{m}{m}$ & 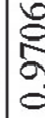 \\
\hline & $\stackrel{0}{0}$ & $\stackrel{P}{\stackrel{P}{+}}$ & $\hat{a}$ & $\begin{array}{l}\Re \\
\infty \\
\infty\end{array} \mid$ & $\stackrel{m}{\stackrel{2}{\sigma}}$ & $\begin{array}{c}R \\
2\end{array}$ & $\frac{\infty}{\infty}$ & \begin{tabular}{l}
$n$ \\
\multirow{5}{*}{} \\
$\infty$
\end{tabular} & $\underset{+\infty}{ \pm}$ & $\underset{\infty}{8}$ & ڤ̊n & 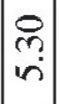 & $\begin{array}{l}\stackrel{\theta}{+} \\
\dot{\nabla}\end{array}$ & I & 1 & 1 & I & I \\
\hline \multirow{2}{*}{ 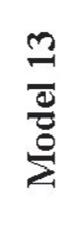 } & $\delta_{0}^{0}$ & 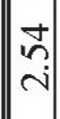 & $\stackrel{\Omega}{\sigma}$ & $\begin{array}{l}0 \\
n \\
0\end{array}$ & ì & $\mid \begin{array}{l}\mathbf{y} \\
\dot{i} \\
\dot{i}\end{array}$ & $\Omega$ & $\stackrel{2}{\Omega}$ & $\stackrel{P}{-}$ & m & ạ. & $\frac{n}{n}$ & $\frac{ \pm}{\dot{r}}$ & {$\left[\begin{array}{l}\infty \\
0 \\
0\end{array} \mid\right.$} & $\mid \begin{array}{c}0 \\
0 \\
\dot{\varphi} \\
\end{array}$ & 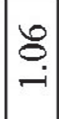 & 2 & \& \\
\hline & $\stackrel{0}{0}$ & $\begin{array}{l}\hat{\sigma} \\
\dot{\gamma}\end{array}$ & $\begin{array}{l}8 \\
8 \\
0\end{array}$ & $\begin{array}{l}\infty \\
i \\
\infty\end{array} \mid$ & $\tilde{n}$ & $\begin{array}{l} \pm \\
\stackrel{2}{2}\end{array}$ & $\mid \begin{array}{l}n \\
\infty \\
\infty\end{array}$ & $\stackrel{m}{\stackrel{m}{+}}$ & $\bar{N}$ & $\begin{array}{l}\stackrel{0}{1} \\
\infty\end{array}$ & $\begin{array}{l}n \\
0 \\
0\end{array}$ & $\begin{array}{l}0 \\
n \\
i n \\
1\end{array}$ & $\begin{array}{l}\vec{f} \\
\dot{f}\end{array}$ & I & 1 & 1 & I & I \\
\hline \multirow{2}{*}{$\frac{\text { J }}{\stackrel{0}{0}}$} & oे & $\stackrel{n}{3}$ & $\stackrel{0}{0}$ & $\begin{array}{l}\stackrel{0}{1} \\
\stackrel{i}{i}\end{array}$ & 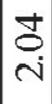 & $\stackrel{2}{0}$ & $\stackrel{m}{m}$ & $\begin{array}{l}\mathcal{O} \\
\stackrel{\circ}{0}\end{array}$ & $\vec{n}$ & $\stackrel{\text { s. }}{-}$ & 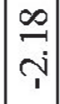 & & $\mid \begin{array}{l}-1 \\
\dot{\varphi} \\
\dot{1}\end{array}$ & $\begin{array}{l}\hat{+} \\
\dot{\varphi} \\
\dot{1}\end{array}$ & $\begin{array}{l}\hat{2} \\
\tilde{1} \\
\hat{\varphi}\end{array}$ & 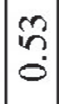 & $\stackrel{?}{3}$ & 8 \\
\hline & $\stackrel{0}{0}$ & $\begin{array}{l}\infty \\
\infty \\
\dot{0}\end{array}$ & $\stackrel{8}{0}$ & $\begin{array}{l}0 \\
\stackrel{0}{ }\end{array}$ & م̊n & $\mid$\begin{tabular}{l}
8 \\
$:$ \\
\hdashline
\end{tabular} & $\begin{array}{l}8 \\
\infty\end{array}$ & $\mid \begin{array}{l}n \\
n \\
\infty\end{array}$ & $\mid \begin{array}{l}0 \\
\infty \\
\infty\end{array}$ & $\underset{\infty}{\infty}$ & $\mid \begin{array}{l}-1 \\
6 \\
0\end{array}$ & $\begin{array}{l}\stackrel{n}{2} \\
i n\end{array}$ & $\mid \begin{array}{l}\infty \\
\stackrel{\infty}{+}\end{array}$ & I & $\mathrm{I}$ & I & I & I \\
\hline \multirow{2}{*}{$\begin{array}{l}\bar{Z} \\
\overline{0} \\
\overline{8}\end{array}$} & $\stackrel{0}{0}$ & $\mid \begin{array}{l}\infty \\
0 \\
0 \\
0\end{array}$ & $\stackrel{+}{\stackrel{0}{0}}$ & $\begin{array}{c}n \\
\tilde{i} \\
\hat{?}\end{array}$ & $\begin{array}{l}0 \\
0 \\
\dot{m}\end{array}$ & $\stackrel{n}{-i}$ & $\begin{array}{l}\hat{\infty} \\
\dot{N}\end{array}$ & $\hat{a}$ & $\begin{array}{l}\vec{y} \\
\dot{r}\end{array}$ & $\vec{N}$ & $\mid \begin{array}{l}0 \\
-1 \\
-1\end{array}$ & $\begin{array}{c}2 \\
\infty \\
0 \\
\dot{0}\end{array}$ & $\stackrel{\vec{n}}{-}$ & $\begin{array}{l}\infty \\
\stackrel{2}{0} \\
0\end{array}$ & 守 & $\begin{array}{l}+ \\
\infty \\
0\end{array}$ & & J \\
\hline & 0 & $\mid \begin{array}{l}9 \\
+ \\
\dot{7}\end{array}$ & : & $\mid \begin{array}{l}0 \\
\infty \\
\infty \\
\infty\end{array}$ & 守 & 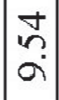 & $\frac{0}{\infty}$ & $\begin{array}{l}+ \\
\infty \\
\infty\end{array}$ & $\underset{\infty}{\stackrel{\circ}{\circ}}$ & $\frac{n}{2}$ & 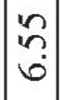 & $\begin{array}{l}\hat{n} \\
\text { ñ }\end{array}$ & 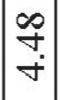 & I & 1 & 1 & I & I \\
\hline \multicolumn{2}{|c|}{$(9 I 0 z-\angle 86 I) u_{G} G$} & $\frac{9}{7}$ & $\underset{6}{6}$ & $\mid \begin{array}{l}\infty \\
\infty \\
\infty\end{array}$ & $\stackrel{2}{2}$ & $\begin{array}{l}9 \\
\dot{9}\end{array}$ & $\stackrel{\sigma}{\circ}$ & $\begin{array}{l}\mathbf{8} \\
\infty\end{array}$ & $\begin{array}{l}n \\
\infty \\
\infty\end{array}$ & $\frac{9}{\infty}$ & 于े & $\begin{array}{c}\bar{m} \\
\dot{n}\end{array}$ & 臬 & & & & & \\
\hline \multicolumn{2}{|c|}{ yłUON } & 劲 & 0ิ & $\stackrel{5}{\Sigma}$ & 穵 & 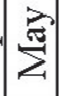 & $\Xi$ & $\Xi$ & $\stackrel{\circ}{\mathrm{z}}$ & 仓 & $\overline{0}$ & $\begin{array}{l}3 \\
2 \\
z\end{array}$ & $\begin{array}{l}8 \\
ٌ \\
\end{array}$ & $\left|\begin{array}{l}1 \\
0 \\
3 \\
z\end{array}\right|$ & $\begin{array}{l}0 \\
00 \\
0 \\
\Sigma\end{array}$ & 式 & $\frac{\vec{\xi}}{\underline{\omega}}$ & \begin{tabular}{|l}
$\frac{1}{2}$ \\
0
\end{tabular} \\
\hline
\end{tabular}




\begin{tabular}{|c|c|c|c|c|c|c|c|c|c|c|c|c|c|c|c|c|c|c|}
\hline \multirow{2}{*}{ 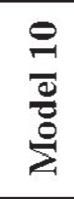 } & $\stackrel{\circ}{\circ}$ & $\stackrel{\text { S. }}{\text { i }}$ & $=$ & $\stackrel{f}{-}$ & $\stackrel{\Re}{\Re}$ & 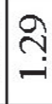 & g) & $\frac{0}{m}$ & $\begin{array}{l}n \\
m\end{array}$ & $\begin{array}{l}\underset{\sim}{\sigma} \\
\text { iv }\end{array}$ & 극 & 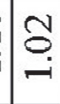 & $\stackrel{2}{-1}$ & $\mid \begin{array}{l}0 \\
0 \\
0 \\
1\end{array}$ & 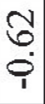 & $\underset{\infty}{+}$ & ๑ & $\begin{array}{l}\infty \\
\stackrel{\infty}{\infty} \\
0\end{array}$ \\
\hline & $\stackrel{0}{0}$ & & $\begin{array}{l}2 \\
n\end{array}$ & 卖 & $\begin{array}{l}8 \\
1\end{array}$ & $\frac{\infty}{\sigma}$ & $\begin{array}{l}8 \\
\infty\end{array}$ & $\underset{\infty}{\infty}$ & $\begin{array}{l}0 \\
\infty\end{array}$ & 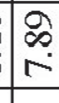 & $\frac{5}{6}$ & 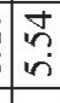 & $\begin{array}{l}n \\
\dot{n} \\
\end{array}$ & 1 & I & I & I & I \\
\hline \multirow{2}{*}{$\frac{a}{\frac{a}{\sigma}}$} & $\delta_{0}^{\circ}$ & & $\frac{2}{2}$ & $\stackrel{ }{=}$ & f & - & $\frac{2}{r}$ & $\infty$ & $\begin{array}{l}F \\
\text { r }\end{array}$ & $\stackrel{\sim}{\sim}$ & $\stackrel{\overbrace{}}{\stackrel{2}{*}}$ & $\stackrel{8}{-}$ & $\underset{-}{\tau}$ & $\begin{array}{l}\Omega \\
\vdots \\
0\end{array}$ & 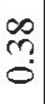 & $\stackrel{J}{-}$ & 2 & \\
\hline & $\stackrel{0}{0}$ & $\begin{array}{l}0 \\
+ \\
+\end{array}$ & $\begin{array}{l}\infty \\
\infty \\
\text { in }\end{array}$ & 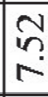 & $\underset{\infty}{\infty}$ & ๙̊ & $\begin{array}{l}7 \\
\infty\end{array}$ & $\begin{array}{l}\stackrel{\sim}{\infty} \\
\infty\end{array}$ & $=$ & $\bar{r}$ & $\underset{6}{0}$ & $\begin{array}{l} \pm \\
n \\
n\end{array}$ & $\stackrel{n}{\sim}$ & 1 & 1 & 1 & I & I \\
\hline \multirow{2}{*}{$\frac{\infty}{\frac{\infty}{\sigma}}$} & $\stackrel{8}{\circ}$ & mֶ? & in & $\frac{n}{m}$ & $\frac{9}{7}$ & $\underset{\sim}{0}$ & $\frac{7}{7}$ & $\ddot{m}$ & $\begin{array}{l}\infty \\
m \\
m\end{array}$ & $\underset{\text { ri }}{\stackrel{S}{0}}$ & $\frac{5}{7}$ & $\vec{n}$ & $\stackrel{0}{8}$ & $\begin{array}{l}2 \\
0 \\
0\end{array}$ & $\begin{array}{l}\stackrel{3}{0} \\
\dot{P}\end{array}$ & ?ִ & $\stackrel{\infty}{\infty}$ & $\stackrel{2}{2}$ \\
\hline & $\stackrel{0}{\Omega}$ & & $\infty$ & ? & 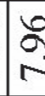 & 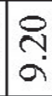 & $\begin{array}{l}\tilde{\sigma} \\
\infty\end{array}$ & $\begin{array}{l}\infty \\
\infty \\
\infty\end{array}$ & $\begin{array}{l}\infty \\
\infty \\
\infty\end{array}$ & $\stackrel{\infty}{\infty}$ & $\frac{6}{6}$ & $\begin{array}{l}m \\
n \\
n\end{array}$ & $\begin{array}{l}n \\
\stackrel{n}{+}\end{array}$ & 1 & I & 1 & I & I \\
\hline \multirow{2}{*}{$\frac{\sigma}{\frac{\sigma}{0}}$} & $\stackrel{\circ}{\circ}$ & $\begin{array}{l}\text { mे } \\
m\end{array}$ & $\sqrt{7}$ & กำ & ñ & 8 & in & 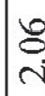 & in & $\stackrel{\text { c }}{-}$ & 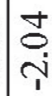 & $\stackrel{\infty}{\infty}$ & 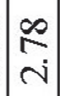 & $\mid \begin{array}{l}0 \\
0 \\
0\end{array}$ & $\begin{array}{l}\tilde{6} \\
0 \\
0\end{array}$ & 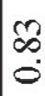 & $\ddot{\hat{\imath}}$ & $\frac{1}{3}$ \\
\hline & $\stackrel{0}{\infty}$ & $\stackrel{m}{r}$ & $\begin{array}{l}\infty \\
\text { in }\end{array}$ & 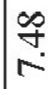 & $\underset{\infty}{2}$ & $\stackrel{\sharp}{\Omega}$ & $\begin{array}{l}R \\
\infty\end{array}$ & $\tilde{m}$ & $\underset{\infty}{\infty}$ & $\underset{\infty}{8}$ & तู & in & $\underset{f}{\tilde{f}}$ & $\mathrm{I}$ & I & I & I & I \\
\hline \multirow{2}{*}{$\frac{b}{\frac{\sigma}{\sigma}}$} & \&े & $\stackrel{8}{\circ}$ & 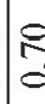 & $\stackrel{n}{\sim}$ & $\tilde{6}$ & $\stackrel{\text { ? }}{\text { ` }}$ & ๙̃ & $\tilde{c}$ & $\frac{1}{2}$ & $\stackrel{ }{=}$ & $\begin{array}{l}\infty \\
\infty \\
r\end{array}$ & $\stackrel{\text { I }}{\sim}$ & 足 & $F$ & $\begin{array}{l}\infty \\
\dot{0}\end{array}$ & ? & $\begin{array}{l}\hat{b} \\
\text { r. }\end{array}$ & $\frac{\infty}{\hat{\Omega}}$ \\
\hline & $\stackrel{0}{0}$ & f & $\infty$ & $\underset{\stackrel{Y}{r}}{\sim}$ & $\underset{\infty}{\infty}$ & $\stackrel{5}{\circ}$ & $\underset{\infty}{-}$ & $\begin{array}{l}f \\
\infty\end{array}$ & $\underset{\infty}{\infty}$ & ${ }_{\infty}^{\infty}$ & ఝి & 卓 & $\stackrel{n}{\sim}$ & $\mathrm{I}$ & I & I & I & I \\
\hline \multirow{2}{*}{$\frac{n}{\frac{0}{0}}$} & $\stackrel{\circ}{\circ}$ & $\stackrel{\infty}{\stackrel{\infty}{-}}$ & $n_{n}^{2}$ & $\stackrel{\leftrightarrow}{n}$ & $\underset{c}{2}$ & $\underset{\text { iे }}{\hat{\theta}}$ & $\frac{m}{r}$ & 8 & ర్ & $\stackrel{8}{\circ}$ & $\stackrel{\Omega}{\longrightarrow}$ & 3 & $\begin{array}{l}8 \\
\dot{\infty} \\
\dot{r}\end{array}$ & $\mid \begin{array}{c}n \\
\tilde{n} \\
0 \\
1\end{array}$ & กี & $\stackrel{2}{2}$ & $\ddot{n}$ & $\stackrel{\infty}{\infty}$ \\
\hline & $\stackrel{0}{0}$ & $\stackrel{\stackrel{\sim}{+}}{\sim}$ & $\delta$ & 守 & $\begin{array}{l}n \\
\infty \\
\infty\end{array}$ & $\begin{array}{l}\swarrow \\
\infty \\
\infty\end{array}$ & $\begin{array}{l}6 \\
\infty \\
\infty\end{array}$ & $\begin{array}{l}0 \\
\infty \\
\infty\end{array}$ & $\begin{array}{l}\text { } \\
\infty\end{array}$ & ${ }_{\infty}$ & શે & $\begin{array}{l}\infty \\
n \\
n\end{array}$ & $\underset{⿱}{\stackrel{y}{+}}$ & 1 & I & I & I & I \\
\hline \multirow{2}{*}{$\frac{ \pm}{\frac{ \pm}{0}}$} & $\delta_{0}^{\circ}$ & ?ִ & $\frac{\mathcal{F}}{7}$ & $\frac{ \pm}{m}$ & $\bar{\gamma}$ & $\hat{\sigma}$ & $\underset{f}{J}$ & $\overline{-}$ & 6 & $\hat{-}$ & $\begin{array}{l}n \\
m \\
m\end{array}$ & 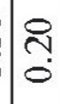 & $\bar{\sigma}$ & 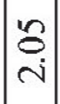 & $\stackrel{+}{\stackrel{n}{\prime}}$ & 9 & nn & $\frac{m}{3}$ \\
\hline & $\stackrel{0}{0}$ & $\stackrel{n}{+}$ & $\begin{array}{l}\infty \\
\text { in }\end{array}$ & ? & $\begin{array}{l}2 \\
2\end{array}$ & $\begin{array}{l}+ \\
\infty \\
\infty\end{array}$ & $\begin{array}{l}J \\
\infty\end{array}$ & $\begin{array}{l}J \\
\infty \\
\infty\end{array}$ & $\frac{\infty}{\infty}$ & $\frac{7}{2}$ & ஸ̂? & in & $\underset{f}{\stackrel{T}{+}}$ & 1 & 1 & 1 & I & I \\
\hline \multirow{2}{*}{$\frac{m}{\frac{\infty}{0}}$} & oे & $\stackrel{8}{\circ}$ & $\frac{9}{r_{1}}$ & $\stackrel{\text { ิ }}{+}$ & a & ঠิ & f & in & ন্ & $\stackrel{8}{6}$ & 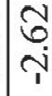 & $\frac{n}{m}$ & $=$ & $\frac{9}{0}$ & $\begin{array}{l}\infty \\
\dot{0}\end{array}$ & $\stackrel{2}{=}$ & $\bar{\curvearrowright}$ & $\stackrel{0}{2}$ \\
\hline & $\stackrel{0}{0}$ & 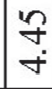 & $\stackrel{\infty}{\sigma}$ & $\stackrel{\substack{N \\
N}}{N}$ & $\begin{array}{l}\infty \\
-\infty\end{array}$ & $\underset{\infty}{\mathbb{N}}$ & $\begin{array}{l}\mathcal{F} \\
\infty\end{array}$ & $\stackrel{\infty}{\underset{N}{N}}$ & $\bar{N}$ & $\hat{\sigma}$ & ํㅜㅇ & $\begin{array}{l}\mathscr{0} \\
i \\
\text { i }\end{array}$ & $\begin{array}{l}\stackrel{2}{n} \\
+\end{array}$ & 1 & 1 & 1 & I & I \\
\hline \multirow{2}{*}{$\frac{N}{\frac{N}{0}}$} & oे & $\frac{D}{P}$ & $\begin{array}{l}\tilde{r} \\
r\end{array}$ & $\stackrel{n}{2}$ & $\ddot{m}$ & $\stackrel{\circ}{\stackrel{0}{N}}$ & $\stackrel{\sim}{\sim}$ & $\begin{array}{l}0 \\
\mathbf{1} \\
\mathbf{i}\end{array}$ & 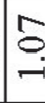 & 导 & @े & के & $\begin{array}{l}\stackrel{\sim}{\sim} \\
\stackrel{N}{2}\end{array}$ & $\begin{array}{l}\infty \\
\infty \\
0\end{array}$ & 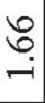 & $\frac{n}{i}$ & $\tilde{\Xi}$ & 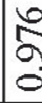 \\
\hline & $\stackrel{0}{0}$ & t. & $\frac{9}{6}$ & 学 & $\frac{\varrho}{\infty}$ & 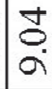 & $\begin{array}{l}2 \\
\infty \\
\infty\end{array}$ & $\begin{array}{l}\mathscr{n} \\
\infty \\
\infty\end{array}$ & 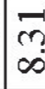 & $\stackrel{\Omega}{\Omega}$ & $\stackrel{0}{\stackrel{0}{0}}$ & $\vec{r}$ & $\stackrel{\stackrel{n}{n}}{+}$ & 1 & I & I & I & I \\
\hline \multirow{2}{*}{$\frac{7}{\frac{0}{\sigma}}$} & oे & $\stackrel{n}{n}$ & $\frac{2}{7}$ & $\begin{array}{l}\infty \\
\stackrel{\infty}{0} \\
\text { in }\end{array}$ & $\vec{J}$ & $\begin{array}{l}\sigma \\
\dot{\sigma} \\
\dot{m}\end{array}$ & f & $\stackrel{n}{9}$ & 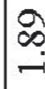 & $\sigma \underline{\sigma}$ & m. & $\stackrel{?}{\stackrel{9}{*}}$ & - & 告 & $\stackrel{\overbrace{}}{\stackrel{\overbrace{}}{*}}$ & 고 & $\hat{\imath}$ & 5 \\
\hline & $\stackrel{8}{0}$ & ले & $\begin{array}{l}5 \\
\text { in }\end{array}$ & 亲 & হ & $\begin{array}{l}\mathscr{2} \\
\infty\end{array}$ & $\begin{array}{l}E \\
\infty\end{array}$ & $\underset{\infty}{\infty}$ & $\underset{\infty}{\sim}$ & م) & $\frac{7}{6}$ & $\begin{array}{l}\infty \\
\stackrel{0}{n}\end{array}$ & $\underset{+}{\sigma}$ & $\mathrm{I}$ & I & I & I & I \\
\hline \multicolumn{2}{|c|}{$(9 I 0 Z-\angle 86 I) u G$} & $\stackrel{P}{+}$ & in & $\underset{0}{0}$ & $\underset{\infty}{\infty}$ & बू & $\Xi$ & $\mid \begin{array}{l}\infty \\
\infty\end{array}$ & $\underset{\infty}{F}$ & $\underset{\infty}{\infty}$ & 응 & $\underset{\text { ir }}{8}$ & $\underset{+}{8}$ & & & & & \\
\hline & & & & $\sum^{ \pm}$ & 定 & $-\frac{3}{\Sigma}$ & 国 & $\Xi$ & 旁 & 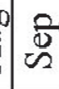 & $-\overrightarrow{0}$ & 完 & $\begin{array}{l}8 \\
\stackrel{d}{日}\end{array}$ & $\mid \begin{array}{l}5 \\
8 \\
3 \\
\end{array}$ & 初 & 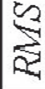 & & $\mid \begin{array}{c}5 \\
0 \\
0\end{array}$ \\
\hline
\end{tabular}




\begin{tabular}{|c|c|c|c|c|c|c|c|c|c|c|c|c|c|c|c|c|c|c|}
\hline \multirow{2}{*}{$\begin{array}{l} \pm \\
\frac{\Xi}{0} \\
\frac{0}{2}\end{array}$} & ஓे & in & $\stackrel{8}{8}$ & $\begin{array}{l}2 \\
0\end{array}$ & 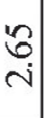 & 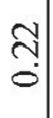 & $\begin{array}{l}\stackrel{n}{n} \\
\text { iv }\end{array}$ & $\begin{array}{c}= \\
\vec{\sim}\end{array}$ & $\begin{array}{l}0 \\
\stackrel{1}{2} \\
\text { in }\end{array}$ & $\begin{array}{c}\vec{J} \\
-i\end{array}$ & ণָ & $\begin{array}{l}0 \\
0 \\
0 \\
0\end{array}$ & 8 & $\begin{array}{l}\frac{J}{0} \\
\dot{0}\end{array}$ & 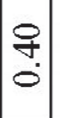 & $\stackrel{8}{-}$ & $\begin{array}{l}\Omega \\
\text { సे }\end{array}$ & $\begin{array}{l}0 \\
5 \\
0 \\
0\end{array}$ \\
\hline & ¿ & $\stackrel{9}{+}$ & $\begin{array}{l}+ \\
\infty \\
i\end{array}$ & $\stackrel{ \pm}{r}$ & $\begin{array}{c}\infty \\
\stackrel{\infty}{\infty} \\
\infty\end{array}$ & 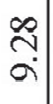 & $\left|\begin{array}{l}\infty \\
\infty \\
\infty\end{array}\right|$ & $\begin{array}{c}N \\
\infty \\
\infty\end{array}$ & $\begin{array}{c}\infty \\
\underset{\infty}{-1}\end{array}$ & ஓ̆ & $\frac{7}{6}$ & $\begin{array}{l}8 \\
\dot{8} \\
\dot{n}\end{array}$ & $\stackrel{N}{\sim}$ & 1 & 1 & 1 & $\mathrm{I}$ & I \\
\hline \multirow{2}{*}{ 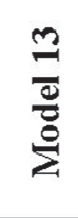 } & $\diamond^{\circ}$ & 군 & $\begin{array}{l}\infty \\
\infty \\
\dot{0}\end{array}$ & $\begin{array}{c}\tilde{3} \\
\dot{0}\end{array}$ & $\vec{i}$ & ঙి & $\begin{array}{l}n \\
\sim \\
i\end{array}$ & $\begin{array}{c}\text { ले } \\
\text { i }\end{array}$ & $\begin{array}{l}\text { in } \\
\text { în }\end{array}$ & $\stackrel{m}{\longrightarrow}$ & \begin{tabular}{l}
0 \\
\hdashline \\
\hdashline
\end{tabular} & $\begin{array}{l}\text { ते. } \\
\text { }\end{array}$ & $\begin{array}{c}\infty \\
\stackrel{\sim}{N}\end{array}$ & $\frac{0}{0}$ & 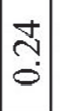 & $\mid \begin{array}{l}\infty \\
0 \\
\dot{0}\end{array}$ & $\stackrel{\Omega}{-}$ & \\
\hline & ¿ & $\begin{array}{c}\mathrm{N} \\
\dot{f}\end{array}$ & $\begin{array}{l}n \\
\infty \\
n \\
n\end{array}$ & 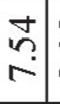 & $\begin{array}{c}\infty \\
\infty \\
\infty \\
\infty\end{array}$ & స̃ & $\stackrel{N}{\infty}$ & $\begin{array}{c}\stackrel{m}{\infty} \\
\infty\end{array}$ & $\frac{9}{\infty}$ & হे & $\begin{array}{c}(7 \\
6\end{array}$ & 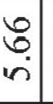 & 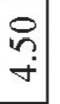 & 1 & 1 & 1 & $\mathrm{I}$ & I \\
\hline \multirow{2}{*}{$\begin{array}{l}\text { U } \\
\frac{0}{0} \\
\frac{0}{2}\end{array}$} & iे & ลึ. & $\begin{array}{l}0 \\
0 \\
0\end{array}$ & $\begin{array}{l}\overrightarrow{3} \\
\hat{i}\end{array}$ & $\begin{array}{l}\stackrel{+}{+} \\
i\end{array}$ & $\begin{array}{l}2 \\
0 \\
0\end{array}$ & $\stackrel{\infty}{2}$ & $\stackrel{2}{=}$ & 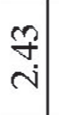 & 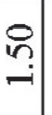 & $\begin{array}{l}\stackrel{0}{7} \\
\div\end{array}$ & 웅. & ઼ָ & 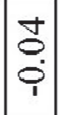 & : & $\mid \begin{array}{c}m \\
\tilde{n} \\
0\end{array}$ & $\begin{array}{l}\infty \\
\infty \\
\circ\end{array}$ & ईू \\
\hline & $\stackrel{\Delta}{0}$ & $\begin{array}{l}\text { वे } \\
\text { t. }\end{array}$ & $\begin{array}{l}n \\
\infty \\
n \\
n\end{array}$ & $\begin{array}{l}\widetilde{\sigma} \\
\stackrel{\sim}{\sim}\end{array}$ & $\begin{array}{c}\stackrel{0}{1} \\
\infty \\
\infty\end{array}$ & సે & $\begin{array}{c}\infty \\
\infty \\
\infty\end{array}$ & $\begin{array}{c}\stackrel{f}{q} \\
\infty\end{array}$ & $\underset{\infty}{\stackrel{\infty}{\infty}}$ & $\begin{array}{l}\infty \\
\end{array}$ & స్ & $\begin{array}{l}\text { n̊ } \\
\text { ñ }\end{array}$ & $\begin{array}{l}\stackrel{9}{+} \\
\dot{+}\end{array}$ & 1 & 1 & 1 & 1 & I \\
\hline \multirow{2}{*}{ 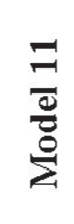 } & oे & $\vec{n}$ & $\begin{array}{c}\infty \\
\\
0\end{array}$ & $\stackrel{8}{0}$ & 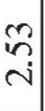 & $\begin{array}{l}n \\
0 \\
0\end{array}$ & $\hat{\tilde{n}}$ & 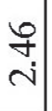 & $\begin{array}{l}\infty \\
\sim \\
\sim \\
\sim\end{array} \mid$ & 극 & 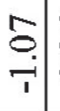 & $\begin{array}{l}0 \\
? \\
? \\
?\end{array}$ & $\stackrel{m}{\mathrm{~N}}$ & $\begin{array}{c}2 \\
0\end{array}$ & 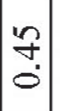 & $\begin{array}{l}\dot{0} \\
\dot{0}\end{array}$ & $\begin{array}{l}\infty \\
\infty \\
ن\end{array}$ & $\frac{2}{2}$ \\
\hline & 8 & $\begin{array}{l}f \\
\dot{\nabla}\end{array}$ & $\begin{array}{l}\infty \\
\infty \\
i\end{array}$ & 命 & $\begin{array}{c}\stackrel{2}{0} \\
\infty\end{array}$ & 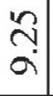 & $\begin{array}{l}2 \\
\infty \\
\infty\end{array}$ & సે & $\begin{array}{c}\infty \\
-\infty \\
\infty\end{array} \mid$ & $\begin{array}{c}8 \\
\infty \\
\infty\end{array}$ & $\begin{array}{l}- \\
6 \\
6\end{array}$ & $\begin{array}{l}\text { Wర } \\
\dot{n}\end{array}$ & $\begin{array}{l}\stackrel{\rho}{\mathrm{f}} \\
\dot{\mathrm{f}}\end{array}$ & 1 & 1 & 1 & 1 & I \\
\hline \multicolumn{2}{|c|}{$(9 I 0 z-\angle 86 I) u_{\sigma}$} & \begin{tabular}{|}
$\vec{n}$ \\
$\vec{\nabla}$
\end{tabular} & $\begin{array}{l}\text { Яิ } \\
\text { ம் }\end{array}$ & \begin{tabular}{l|l}
8 \\
$\sim$ \\
\end{tabular} & $\begin{array}{c}\text { ల్ } \\
\infty\end{array}$ & ભે & 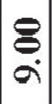 & $\begin{array}{l}\overrightarrow{\mathrm{n}} \\
\infty\end{array}$ & $\begin{array}{c}\mathcal{f} \\
\underset{\infty}{0}\end{array}$ & $\underset{\infty}{\stackrel{\theta}{\infty}}$ & $\begin{array}{l}\text { 영 } \\
6\end{array}$ & $\begin{array}{c}\overrightarrow{6} \\
\dot{1}\end{array}$ & $\begin{array}{l}\stackrel{8}{\sigma} \\
\stackrel{+}{+}\end{array}$ & & & & & \\
\hline \multicolumn{2}{|c|}{ чриоW } & 击 & & $\stackrel{\vec{z}}{\Sigma}$ & 完 & 穵 & $\Xi$ & $\Xi$ & 兊 & ठे & $\overrightarrow{0}$ & $\begin{array}{l}3 \\
\vdots \\
z\end{array}$ & \begin{tabular}{l}
$\mathscr{\Xi}$ \\
\hdashline
\end{tabular} & $\mid \begin{array}{c}1 \\
\frac{1}{2} \\
\frac{2}{2}\end{array}$ & $\mid \begin{array}{l}1 \\
\frac{1}{20} \\
\frac{9}{2}\end{array}$ & 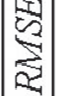 & 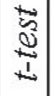 & $\frac{1}{15}$ \\
\hline
\end{tabular}




\begin{tabular}{|c|c|c|c|c|c|c|c|c|c|c|c|c|c|c|c|c|c|c|}
\hline \multirow{2}{*}{$\begin{array}{l}\text { 을 } \\
\frac{0}{0} \\
\sum_{2}^{\circ}\end{array}$} & $\dot{\theta}_{0}^{\circ}$ & $\stackrel{n}{0}$ & $\begin{array}{l}\infty \\
\stackrel{n}{\circ}\end{array}$ & $\stackrel{m}{\longrightarrow}$ & $\begin{array}{l}= \\
\dot{r}\end{array}$ & $\begin{array}{c}0 \\
0 \\
0\end{array} \mid$ & $\begin{array}{l}\text { ஓे } \\
\text { ㄱ. }\end{array}$ & $\begin{array}{l}\vec{b} \\
\dot{\sim}\end{array} \mid$ & $\begin{array}{c}\hat{0} \\
\dot{m}\end{array}$ & $\begin{array}{c}\overrightarrow{0} \\
\stackrel{i}{ }\end{array}$ & $\begin{array}{c}n \\
7 \\
i\end{array}$ & $\begin{array}{c}\infty \\
\stackrel{\infty}{0} \\
0\end{array}$ & $\underset{g}{q}$ & $\stackrel{+}{m}$ & $\begin{array}{l}\overrightarrow{1} \\
\overrightarrow{1} \\
\end{array}$ & $\stackrel{\leftrightarrow}{\circ}$ & $\stackrel{\bar{m}}{\mathrm{c}}$ & 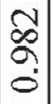 \\
\hline & ڤั & $\begin{array}{l}\text { f } \\
\text { f }\end{array}$ & $\begin{array}{l}7 \\
\text { in }\end{array}$ & $\begin{array}{l}\tilde{m} \\
\stackrel{n}{*}\end{array}$ & $\begin{array}{l}\mathscr{m} \\
\infty \\
\infty\end{array}$ & $\mid$\begin{tabular}{|c|}
$\infty$ \\
$n$ \\
$\infty$ \\
$\infty$
\end{tabular} & ִִ & $\begin{array}{l}8 \\
-1 \\
\end{array}$ & ?ִ & $\begin{array}{l}m \\
r \\
R\end{array}$ & $\begin{array}{l}6 \\
i n\end{array}$ & $\begin{array}{l}\tilde{n} \\
\dot{f}\end{array}$ & $\begin{array}{l}\Xi \\
\dot{\nabla}\end{array}$ & 1 & I & 1 & 1 & I \\
\hline \multirow{2}{*}{$\frac{a}{\frac{0}{0}}$} & oे & مُ & $\frac{2}{0}$ & $\stackrel{f}{\circ}$ & ๙ & {$\left[\begin{array}{c}\infty \\
n \\
0\end{array} \mid\right.$} & 足 & $\begin{array}{c}\vec{\delta} \\
\stackrel{\sim}{i}\end{array} \mid$ & $\begin{array}{l}\mathrm{S} \\
\mathrm{i}\end{array}$ & $\overline{-}$ & $\begin{array}{c}q \\
-i \\
i\end{array}$ & $\begin{array}{c}\infty \\
\infty \\
0\end{array}$ & $\begin{array}{l}\infty \\
\dot{0}\end{array}$ & $\stackrel{m}{\stackrel{m}{\circ}}$ & $\stackrel{9}{\stackrel{9}{0}}$ & $\stackrel{5}{\infty}$ & 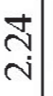 & 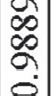 \\
\hline & 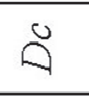 & \begin{tabular}{|l|l|} 
\\
+ \\
+ \\
+
\end{tabular} & $\begin{array}{l}\stackrel{f}{f} \\
\text { in }\end{array}$ & $\begin{array}{l}\hat{m} \\
\stackrel{n}{-}\end{array}$ & $\begin{array}{l}3 \\
\infty \\
\infty\end{array}$ & $\begin{array}{c}8 \\
\infty \\
\infty\end{array} \mid$ & হ. & \begin{tabular}{|c|} 
\\
6 \\
2 \\
\end{tabular} & 吕 & $\begin{array}{l}5 \\
\end{array}$ & $\begin{array}{l}\hat{6} \\
\text { in }\end{array}$ & $\begin{array}{l}n \\
n \\
\sim\end{array}$ & $\begin{array}{l}0 \\
-1 \\
\end{array}$ & 1 & I & 1 & 1 & I \\
\hline \multirow{2}{*}{$\frac{\infty}{\frac{\infty}{0}}$} & o̊ & 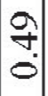 & $\stackrel{\infty}{\stackrel{\infty}{n}}$ & $\mid \begin{array}{l}\sigma \\
\sigma \\
-i\end{array}$ & $\begin{array}{c}m \\
m\end{array}$ & $\begin{array}{c}\stackrel{\circ}{\infty} \\
\stackrel{\circ}{0}\end{array}$ & $\begin{array}{l}\widehat{N} \\
\text { ָे }\end{array}$ & $\begin{array}{c}\vec{J} \\
\dot{\sim} \\
\dot{\gamma}\end{array}$ & $\frac{a}{m}$ & $\begin{array}{c} \pm \\
\stackrel{\sim}{\sim}\end{array}$ & $\begin{array}{c} \pm \\
\\
\end{array}$ & $\stackrel{r}{\vec{f}}$ & $\stackrel{8}{6}$ & 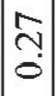 & 고 & 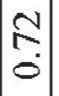 & $\underset{\sim}{\mathbb{N}}$ & ळ్ \\
\hline & 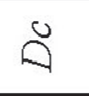 & $\begin{array}{l}\infty \\
+ \\
+ \\
+\end{array}$ & $\begin{array}{l}\text { ت } \\
\text { in }\end{array}$ & 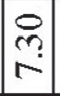 & $\underset{\infty}{0}$ & $\begin{array}{c}\infty \\
n \\
\infty \\
\infty\end{array} \mid$ & 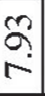 & $\mid \begin{array}{c}\hat{\sigma} \\
-\end{array}$ & חָ & $\begin{array}{c}N \\
N\end{array}$ & $\begin{array}{l}6 \\
i \\
i\end{array}$ & in & $\stackrel{m}{\stackrel{9}{+}}$ & $\mathrm{I}$ & I & I & 1 & I \\
\hline \multirow{2}{*}{$\frac{n}{2}$} & oे & 苂 & $\begin{array}{l}- \\
0 \\
0\end{array}$ & 吢 & $\frac{\sim}{m}$ & ஓे & $\begin{array}{c}3 \\
\dot{r} \\
r\end{array}$ & $\begin{array}{l}\vec{b} \\
\dot{\sim}\end{array} \mid$ & $\hat{\widehat{a}}$ & 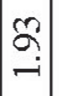 & $\begin{array}{l}n \\
7 \\
1\end{array}$ & $\begin{array}{l}\stackrel{0}{\infty} \\
\circ\end{array}$ & $\stackrel{\infty}{\Omega}$ & $\left(\begin{array}{c}n \\
\hdashline\end{array}\right.$ & $\begin{array}{l}1 \\
8 \\
0\end{array}$ & $\begin{array}{c}\mathscr{C} \\
\hat{O} \\
\dot{0}\end{array}$ & $\begin{array}{l}\Omega \\
\text { in }\end{array}$ & $\stackrel{m}{2}$ \\
\hline & 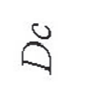 & $\stackrel{\vartheta}{f}$ & $\begin{array}{l}\stackrel{f}{f} \\
\text { in }\end{array}$ & $\stackrel{\bar{m}}{r}$ & $\underset{\infty}{\infty}$ & $\left|\begin{array}{c}0 \\
n \\
\infty \\
\infty\end{array}\right|$ & $\stackrel{2}{\Upsilon}$ & $\mid \begin{array}{c}-6 \\
-\end{array}$ & 吕 & $\frac{\pi}{5}$ & $\begin{array}{l}P \\
\text { in }\end{array}$ & $\left|\begin{array}{l}n \\
\tilde{f} \\
\dot{\gamma}\end{array}\right|$ & 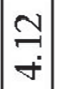 & 1 & I & 1 & I & I \\
\hline \multirow{2}{*}{$\frac{0}{\frac{0}{g}}$} & ò & $\stackrel{t}{\stackrel{0}{0}}$ & $\stackrel{N}{\circ}$ & $\stackrel{R}{-}$ & $\begin{array}{c}\tilde{O} \\
\dot{\theta}\end{array}$ & $\left|\begin{array}{l}2 \\
-2\end{array}\right|$ & $\begin{array}{l}3 \\
\\
\ddot{n}\end{array}$ & $\stackrel{n}{-}$ & $\begin{array}{l}2 \\
i\end{array}$ & $F$ & $\begin{array}{l}\text { ñ } \\
\text { กิ }\end{array}$ & $F$ & $\stackrel{n}{n}$ & $\stackrel{\sim}{n}$ & $\begin{array}{l}\tilde{\sigma} \\
\dot{0}\end{array}$ & $\mid \begin{array}{c}\infty \\
\stackrel{\sim}{-}\end{array}$ & $\begin{array}{c}\exists \\
\dot{m}\end{array}$ & $\stackrel{1}{\hat{s}}$ \\
\hline & $\stackrel{\Delta}{\Delta}$ & 守 & $\begin{array}{l}\stackrel{f}{+} \\
\text { in }\end{array}$ & શุ. & $\underset{\infty}{\stackrel{D}{0}}$ & $\left|\begin{array}{l}8 \\
n \\
\infty\end{array}\right|$ & $\stackrel{+}{2}$ & $\left|\begin{array}{c}9 \\
0 \\
-1\end{array}\right|$ & $\begin{array}{l}\text { ñ } \\
\\
r\end{array}$ & $\begin{array}{l}0 \\
2 \\
-1\end{array}$ & $\begin{array}{l}n \\
\text { in }\end{array}$ & $\mid \begin{array}{l}\vec{n} \\
\dot{\gamma}\end{array}$ & $\begin{array}{l} \pm \\
\dot{\nabla}\end{array}$ & $\mathrm{I}$ & I & 1 & 1 & I \\
\hline \multirow{2}{*}{$\frac{n}{\frac{1}{0}}$} & $\stackrel{\circ}{0}$ & $\begin{array}{c}- \\
0 \\
0\end{array}$ & $\stackrel{\leftrightarrow}{0}$ & $\left|\begin{array}{l} \pm \\
\infty \\
\dot{i}\end{array}\right|$ & $\begin{array}{l}\vec{B} \\
\dot{m}\end{array}$ & $\left|\begin{array}{c}n \\
\tilde{n}\end{array}\right|$ & $\begin{array}{l}2 \\
\hat{m}\end{array}$ & $\begin{array}{l}\stackrel{\sim}{0} \\
\stackrel{\sim}{i}\end{array} \mid$ & $\vec{i}$ & $F$ & $\begin{array}{l}\stackrel{0}{1} \\
\text { ì }\end{array}$ & $\begin{array}{l}+ \\
\infty \\
0 \\
0\end{array}$ & 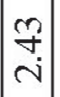 & $\mid \begin{array}{c}n \\
\dot{0} \\
i\end{array}$ & 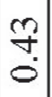 & $\stackrel{n}{a}$ & $\overrightarrow{\mathrm{N}}$ & $\begin{array}{l}\stackrel{2}{\infty} \\
\stackrel{\alpha}{\alpha}\end{array}$ \\
\hline & $\stackrel{\Delta}{\Delta}$ & $\begin{array}{l}\text { f } \\
\end{array}$ & $\begin{array}{l}\text { 寸 } \\
\stackrel{\text { in }}{ }\end{array}$ & $\begin{array}{l}\bar{N} \\
-\end{array}$ & ঐ. & \begin{tabular}{|c|}
2 \\
$\ddots$ \\
$\infty$
\end{tabular} & $\begin{array}{l}\infty \\
\infty \\
\infty \\
\end{array}$ & \begin{tabular}{|l|}
6 \\
6 \\
-
\end{tabular} & ח? & $\begin{array}{l} \\
2 \\
-1\end{array}$ & $\begin{array}{l}\Re \\
i \\
i\end{array}$ & $\begin{array}{l}n \\
n \\
q\end{array}$ & $\begin{array}{l}0 \\
\stackrel{1}{+} \\
\end{array}$ & 1 & I & 1 & I & I \\
\hline \multirow{2}{*}{$\frac{ \pm}{\frac{d}{g}}$} & $\delta_{0}^{\circ}$ & ભે & $\underset{0}{\stackrel{0}{0}}$ & $\mid \begin{array}{c}0 \\
\stackrel{n}{i} \\
\mid\end{array}$ & $\stackrel{n}{m}$ & $\begin{array}{c}\infty \\
\infty \\
\dot{N}\end{array} \mid$ & 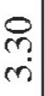 & શ. & $\stackrel{\vec{n}}{i}$ & $\left|\begin{array}{c}-6 \\
-i\end{array}\right|$ & $\begin{array}{l}\hat{\sigma} \\
\hat{i} \\
\dot{1}\end{array}$ & $\stackrel{?}{0}$ & $\begin{array}{c}\stackrel{P}{ } \\
\dot{m}\end{array} \mid$ & $\left.\mid \begin{array}{c}0 \\
\\
-1\end{array}\right]$ & $\begin{array}{l}\stackrel{0}{ } \\
-i\end{array}$ & $\stackrel{m}{-1}$ & $m$ & $\stackrel{N}{\Omega}$ \\
\hline & ¿ & 导 & $\begin{array}{l}\text { 守 } \\
\text { in }\end{array}$ & 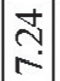 & $\bar{\infty}$ & $\begin{array}{c}7 \\
\overline{0} \\
\infty\end{array} \mid$ & $\stackrel{\wp}{\Omega}$ & $\left|\begin{array}{c}0 \\
0 \\
-1\end{array}\right|$ & م. & $\begin{array}{c}0 \\
2 \\
2\end{array}$ & $\begin{array}{l}\stackrel{6}{2} \\
\text { in }\end{array}$ & $\begin{array}{l}n \\
\dot{f}\end{array}$ & $\begin{array}{l}\mathscr{\wp} \\
\dot{+}\end{array}$ & 1 & 1 & I & I & I \\
\hline \multirow{2}{*}{$\frac{m}{\frac{D}{g}}$} & $\delta_{0}^{\circ}$ & $\ddot{\infty}$ & $\stackrel{\overbrace{}}{\rightarrow}$ & $\left|\begin{array}{c}\infty \\
\stackrel{\infty}{ } \\
r\end{array}\right|$ & 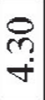 & 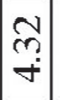 & $\begin{array}{l}\infty \\
\infty \\
\dot{+}\end{array}$ & $\begin{array}{c}J \\
-i\end{array}$ & 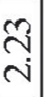 & $\stackrel{n}{-}$ & $\begin{array}{c}\stackrel{n}{r} \\
\text { ri }\end{array}$ & \begin{tabular}{c}
$n$ \\
\hdashline \\
0
\end{tabular} & $\begin{array}{l}n \\
\infty \\
\dot{0}\end{array}$ & $\stackrel{2}{a}$ & $\begin{array}{c}\infty \\
\infty \\
0\end{array}$ & ڤ̊. & 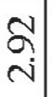 & $\frac{1}{a}$ \\
\hline & $\tilde{a}$ & fo & ñ & $\frac{\infty}{\frac{\infty}{n}}$ & $\Omega$ & 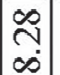 & \begin{tabular}{c}
$\stackrel{\infty}{\infty}$ \\
\hdashline
\end{tabular} & $\begin{array}{l}P \\
r\end{array}$ & & $\begin{array}{c}\infty \\
\stackrel{2}{r}\end{array}$ & $\begin{array}{l}2 \\
\text { in }\end{array}$ & $\stackrel{n}{f}$ & $\begin{array}{l}0 \\
+ \\
\dot{0}\end{array}$ & 1 & 1 & 1 & I & I \\
\hline \multirow{2}{*}{$\frac{\frac{N}{0}}{\sum_{0}^{\circ}}$} & $\partial_{0}^{\circ}$ & $\begin{array}{l}n \\
0 \\
0 \\
1\end{array}$ & $\frac{\Omega}{7}$ & $\stackrel{ \pm}{\stackrel{ \pm}{\sim}}$ & $\stackrel{m}{\stackrel{m}{N}}$ & $\left.\begin{array}{c}\stackrel{c}{\sim} \\
r\end{array}\right]$ & $\begin{array}{c}\stackrel{N}{N} \\
\stackrel{r}{*}\end{array}$ & $\begin{array}{c}5 \\
\infty \\
0\end{array}$ & $\stackrel{\vec{m}}{\stackrel{\sim}{-}}$ & $\bar{\Omega}$ & $\begin{array}{l}8 \\
\text { ஸे } \\
i\end{array}$ & $\mid \begin{array}{l}0 \\
2 \\
0 \\
1\end{array}$ & $\begin{array}{l}\circ \\
\stackrel{2}{2}\end{array}$ & $\begin{array}{c}\exists \\
\dot{\sigma} \\
\end{array}$ & $\stackrel{8}{8}$ & $\mid$\begin{tabular}{c}
8 \\
\hdashline \\
$-i$
\end{tabular} & $\stackrel{n}{\sim}$ & $\stackrel{2}{\hat{a}}$ \\
\hline & $\ddot{a}$ & $\stackrel{\leftrightarrow}{\rightarrow}$ & $\begin{array}{l}n \\
n \\
n\end{array}$ & $\mid \begin{array}{l}0 \\
N \\
\end{array}$ & $\underset{\infty}{\stackrel{8}{0}}$ & $\left|\begin{array}{l}n \\
m \\
\infty\end{array}\right|$ & $\begin{array}{l}+ \\
\vdots \\
\end{array}$ & $\begin{array}{l} \pm \\
2\end{array}$ & 站 & $\left|\begin{array}{c}\infty \\
\infty \\
\sim\end{array}\right|$ & $\begin{array}{c}m \\
r i\end{array}$ & $\begin{array}{c}\hat{b} \\
\dot{+}\end{array} \mid$ & $\left|\begin{array}{l}\infty \\
\stackrel{0}{+} \\
\dot{+}\end{array}\right|$ & 1 & I & 1 & 1 & I \\
\hline \multirow{2}{*}{$\frac{\bar{D}}{\frac{D}{E}}$} & $\partial_{0}^{0}$ & $\begin{array}{l}\text { के } \\
0\end{array}$ & $\stackrel{+}{m}$ & $\overrightarrow{\hat{\sigma}}$ & $\begin{array}{l}\stackrel{g}{+} \\
\dot{\sim}\end{array}$ & $\begin{array}{c}\sim \\
\sim \\
\forall\end{array}$ & $\underset{\sim}{\stackrel{\sim}{+}}$ & $\begin{array}{l}\infty \\
\stackrel{\infty}{\sim} \\
\sim\end{array} \mid$ & 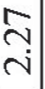 & $\begin{array}{l}9 \\
-i\end{array}$ & $\begin{array}{c}0 \\
\infty \\
-1 \\
-1\end{array}$ & $\begin{array}{l}0 \\
0 \\
\dot{1}\end{array}$ & 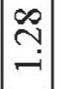 & $\stackrel{m}{-2}$ & $\begin{array}{l}\infty \\
\\
-\end{array}$ & $\left|\begin{array}{c}\hat{O} \\
\stackrel{\sim}{i}\end{array}\right|$ & $\begin{array}{l}\infty \\
\infty \\
\dot{\sim}\end{array}$ & $\stackrel{+}{\stackrel{5}{0}}$ \\
\hline & ڤ & f̊ & ñ & 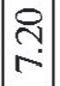 & $\frac{\alpha}{r}$ & 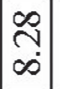 & 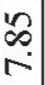 & 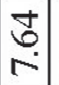 & 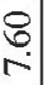 & $\begin{array}{l}2 \\
i\end{array}$ & in & $\begin{array}{c}1 \\
\sigma \\
+\end{array} \mid$ & \begin{tabular}{l}
$n$ \\
\hdashline
\end{tabular} & $\mathrm{I}$ & I & 1 & I & I \\
\hline \multicolumn{2}{|c|}{$(9 I 0 Z-\angle 86 I) u G$} & $\stackrel{8}{+0}$ & $\underset{ن}{7}$ & \begin{tabular}{c}
\multirow{y}{Y}{} \\
\end{tabular} & సે & $\left|\begin{array}{c}1 \\
\infty \\
\infty\end{array}\right|$ & స్త్ & $\begin{array}{l}\infty \\
\infty \\
-1\end{array}$ & \begin{tabular}{l}
$\infty$ \\
\hdashline \\
\end{tabular} & $\begin{array}{l}\infty \\
\infty \\
-1\end{array}$ & $\begin{array}{c}\overline{6} \\
\text { मी }\end{array}$ & 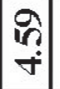 & $\begin{array}{l}\stackrel{\mathrm{N}}{+} \\
+\end{array}$ & & & & & \\
\hline \multicolumn{2}{|c|}{ Y740IN } & & 0 & $\stackrel{5}{\Sigma}$ & 完 & $\sum_{i}^{3}$ & $\Xi$ & $\Xi$ & $\stackrel{00}{\mathrm{Z}_{4}}$ & 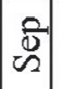 & $\overrightarrow{0}$ & $\begin{array}{l}3 \\
2 \\
z\end{array}$ & $\begin{array}{l}0 \\
\ddot{y} \\
0\end{array}$ & $\mid \begin{array}{l}{\left[\begin{array}{c}1 \\
0 \\
z \\
z\end{array}\right.}\end{array}$ & ô & 这 & 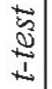 & $\begin{array}{l}\frac{1}{5} \\
\frac{2}{2} \\
2\end{array}$ \\
\hline
\end{tabular}




\begin{tabular}{|c|c|c|c|c|c|c|c|c|c|c|c|c|c|c|c|c|c|c|}
\hline \multirow{2}{*}{$\frac{ \pm}{\frac{D}{J}}$} & oे & 足 & $\begin{array}{l}\infty \\
\infty \\
0\end{array}$ & 8 & $\bar{\Omega}$ & g & $\stackrel{n}{\stackrel{n}{*}}$ & $\tilde{m}$ & $\begin{array}{l}\mathcal{N} \\
\sim \\
\dot{N}\end{array}$ & 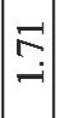 & $\begin{array}{l}\exists \\
\stackrel{1}{7}\end{array}$ & స̦. & $\stackrel{-}{0}$ & $\stackrel{+}{n}$ & $\stackrel{ \pm}{ \pm}$ & $\begin{array}{l}\mathscr{2} \\
\stackrel{\vdots}{0}\end{array}$ & & : \\
\hline & $\stackrel{\circ}{\circ}$ & $\stackrel{9}{\stackrel{9}{f}}$ & $\begin{array}{l}\stackrel{g}{9} \\
\text { in }\end{array}$ & $m$ & 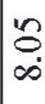 & $\vec{\sigma}$ & $\widehat{a}$ & 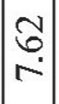 & $\begin{array}{l}0 \\
n \\
r\end{array}$ & $\begin{array}{l}2 \\
r\end{array}$ & $\begin{array}{l}\infty \\
0 \\
i\end{array}$ & $\mid \begin{array}{c}\infty \\
\sim \\
\dot{\gamma}\end{array}$ & $\underset{\sim}{\stackrel{m}{\sigma}}$ & I & 1 & I & I & I \\
\hline \multirow{2}{*}{$\frac{m}{\frac{m}{2}}$} & o̊ & $\stackrel{m}{=}$ & $\begin{array}{l}\infty \\
\dot{0}\end{array}$ & 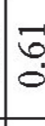 & $\begin{array}{l}\infty \\
\stackrel{\infty}{i}\end{array}$ & 导 & $\stackrel{i}{n}$ & 고 & $\frac{d}{i}$ & $\left(\begin{array}{c}\sigma \\
-\end{array}\right.$ & $\frac{0}{T}$ & 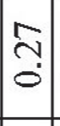 & $\frac{n}{n}$ & $\begin{array}{l}N \\
? \\
0\end{array}$ & 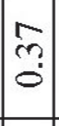 & $\stackrel{8}{\circ}$ & $\stackrel{\infty}{-}$ & के \\
\hline & $\ddot{0}$ & 年 & 守 & $m$ & $\begin{array}{l}\mathscr{8} \\
\infty \\
\infty\end{array}$ & $\vec{b}$ & $\stackrel{\Omega}{\circ}$ & \begin{tabular}{l}
\multirow{0}{0}{} \\
$r$
\end{tabular} & $\stackrel{n}{n}$ & $\begin{array}{l}0 \\
2 \\
2\end{array}$ & $\begin{array}{l}0 \\
n \\
n \\
n\end{array}$ & $\left|\begin{array}{l}2 \\
\sigma \\
\dot{\gamma}\end{array}\right|$ & $\stackrel{m}{\dot{\sigma}}$ & I & $\mathrm{I}$ & I & I & I \\
\hline \multirow{2}{*}{$\frac{\text { I }}{\frac{d}{\delta}}$} & $\stackrel{\circ}{\circ}$ & ?. & $F$ & $\because$ & $\begin{array}{l}\infty \\
\text { ij }\end{array}$ & f & $\begin{array}{l}\tilde{C} \\
\stackrel{N}{*}\end{array}$ & 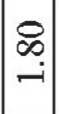 & $\begin{array}{l}\infty \\
i \\
i\end{array}$ & 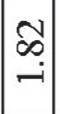 & $\frac{\text { 워 }}{\mathrm{i}}$ & $\begin{array}{l}n \\
0 \\
0\end{array}$ & - & $\stackrel{m}{0}$ & $\overline{1}$ & $\begin{array}{l}\infty \\
n \\
0\end{array}$ & $\stackrel{8}{-}$ & : \\
\hline & $\stackrel{0}{0}$ & $\begin{array}{l}\infty \\
\text { f } \\
\end{array}$ & $\begin{array}{l}f \\
\text { ñ } \\
\end{array}$ & $m$ & $\begin{array}{l}2 \\
\infty \\
\infty\end{array}$ & $\bar{\sigma}$ & 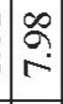 & $\begin{array}{l}6 \\
- \\
\end{array}$ & $\begin{array}{l}0 \\
r \\
r\end{array}$ & $\begin{array}{l}n \\
r \\
i\end{array}$ & $\begin{array}{l}8 \\
\text { in } \\
\end{array}$ & $\begin{array}{l}0 \\
n \\
\forall \\
\end{array}$ & $\begin{array}{l}0 \\
+ \\
+\end{array}$ & I & I & I & I & I \\
\hline \multirow{2}{*}{$\bar{z}$} & $o^{\circ}$ & $\stackrel{n}{n}$ & $\stackrel{R}{\circ}$ & fo & $\begin{array}{l}\mathcal{N} \\
\text { ․ }\end{array}$ & $\stackrel{\mathcal{Y}}{0}$ & 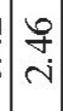 & $\begin{array}{c}\stackrel{\sim}{f} \\
\stackrel{\sim}{*}\end{array}$ & $\begin{array}{l}n \\
i\end{array}$ & 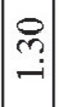 & $\left|\begin{array}{l}\infty \\
0 \\
\hdashline \\
\end{array}\right|$ & $\begin{array}{c}1 \\
\hat{T} \\
\vdots \\
\end{array}$ & 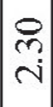 & $\begin{array}{l}0 \\
0 \\
0\end{array}$ & $\begin{array}{c}m \\
\mathfrak{n} \\
0\end{array}$ & $\begin{array}{l}\infty \\
\stackrel{2}{0}\end{array}$ & $\begin{array}{l}\stackrel{8}{\circ} \\
\dot{r}\end{array}$ & $\begin{array}{l}0 \\
\ddot{8} \\
0 \\
0\end{array}$ \\
\hline & $\stackrel{\Delta}{\Delta}$ & $\stackrel{m}{\stackrel{f}{f}}$ & 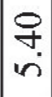 & m & io & $\bar{\sigma}$ & $\begin{array}{l}8 \\
\infty \\
\infty\end{array}$ & $\begin{array}{c}\sigma \\
\sigma \\
\sim\end{array}$ & $\begin{array}{l}\infty \\
n \\
n\end{array}$ & $\begin{array}{l}2 \\
2\end{array}$ & $\begin{array}{l}6 \\
i \\
\end{array}$ & $\left|\begin{array}{l}\mathscr{8} \\
\dot{+}\end{array}\right|$ & $\stackrel{\circ}{\stackrel{9}{+}}$ & I & 1 & I & I & I \\
\hline \multicolumn{2}{|c|}{$(9 I 0 z-\angle 86 I) u_{G}$} & $\stackrel{\text { p }}{+}$ & $\begin{array}{l}7 \\
\dot{8}\end{array}$ & $\frac{\gamma}{r}$ & సે & $\begin{array}{l}12 \\
\infty\end{array}$ & $\underset{\infty}{\underset{\infty}{\infty}}$ & $\mid \begin{array}{l}\infty \\
\dot{r}\end{array}$ & $\begin{array}{l}\infty \\
⿱ \\
r\end{array}$ & $\begin{array}{l}\infty \\
\infty \\
\end{array}$ & $\begin{array}{l}\overline{6} \\
\dot{10}\end{array}$ & in & ণิ & & & & & \\
\hline \multicolumn{2}{|c|}{ чңบоО } & & D & $\stackrel{ \pm}{z}$ & 宗 & $\sum^{3}$ & $\Xi$ & $\Xi$ & $\stackrel{\infty}{\Xi}$ & 逗 & $\overrightarrow{0}$ & $\mid \begin{array}{l}3 \\
0 \\
z\end{array}$ & $\mathscr{8}$ & $\frac{1}{10}$ & \begin{tabular}{|l}
$\frac{1}{10}$ \\
0 \\
$\frac{3}{z}$
\end{tabular} & $\frac{\sqrt{3}}{3}$ & $\begin{array}{l}\frac{\omega}{2} \\
\stackrel{\omega}{ \pm}\end{array}$ & $\begin{array}{l}\frac{1}{0} \\
0 \\
2\end{array}$ \\
\hline
\end{tabular}




\begin{tabular}{|c|c|c|c|c|c|c|c|c|c|c|c|c|c|c|c|c|c|c|}
\hline \multirow{2}{*}{ 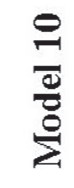 } & $\rho_{0}^{\circ}$ & กิ: & $\begin{array}{l}\stackrel{0}{0} \\
\vdots \\
0\end{array}$ & $\stackrel{+}{\stackrel{H}{0}}$ & $\overrightarrow{\mathrm{N}}$ & $\stackrel{?}{\stackrel{?}{\circ}}$ & 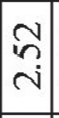 & $\stackrel{m}{\mathrm{~m}}$ & $\left|\begin{array}{l}n \\
6 \\
i\end{array}\right|$ & 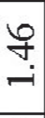 & $\begin{array}{c}0 \\
\cdots \\
7\end{array}$ & $\begin{array}{l}3 \\
0 \\
0 \\
\end{array}$ & $\begin{array}{c} \pm \\
\text { - } \\
\end{array}$ & & $\stackrel{?}{\stackrel{9}{\circ}}$ & 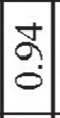 & $\begin{array}{l}2 \\
\text { ì }\end{array}$ & $\begin{array}{l} \pm \\
\infty \\
0 \\
0\end{array}$ \\
\hline & 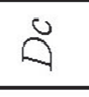 & $\begin{array}{l} \\
\text { f } \\
\end{array}$ & $\begin{array}{l}n \\
\dot{y} \\
\dot{\forall}\end{array}$ & $\begin{array}{c}n \\
0 \\
\end{array}$ & $\begin{array}{l}0 \\
\vdots \\
0\end{array}$ & $\begin{array}{l}9 \\
0 \\
\infty\end{array}$ & $\begin{array}{c}7 \\
\infty \\
\infty\end{array}$ & $\begin{array}{l}n \\
6 \\
6\end{array}$ & $\begin{array}{c} \pm \\
\\
\end{array}$ & $\begin{array}{l}? \\
0 \\
0\end{array}$ & $\begin{array}{c}\text { Na } \\
\text { in } \\
\end{array}$ & $\begin{array}{l}\hat{\sigma} \\
\dot{+}\end{array}$ & $\begin{array}{l}\infty \\
0 \\
\dot{y} \\
\end{array}$ & I & 1 & I & I & I \\
\hline \multirow{2}{*}{$\frac{a}{\frac{0}{0}}$} & $\partial_{0}^{\circ}$ & @: & $\begin{array}{c}n \\
\vdots \\
0 \\
0\end{array}$ & 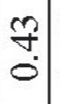 & $\begin{array}{l}\stackrel{0}{N} \\
i\end{array}$ & ڤ̊? & (ñ & $\begin{array}{l}\text { त̂ } \\
\text { ¿ }\end{array}$ & $\begin{array}{l} \pm \\
\dot{N}\end{array}$ & $\begin{array}{l}8 \\
- \\
-\end{array}$ & ָิ & ֻُ & ำ & مֶ & $\begin{array}{l}+ \\
0 \\
0\end{array}$ & {$\left[\begin{array}{c}\infty \\
\infty \\
0 \\
0\end{array}\right]$} & $\begin{array}{l}\text { ले } \\
\text { సे }\end{array}$ & 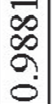 \\
\hline & $\stackrel{0}{0}$ & वे & $\begin{array}{l}\mathscr{2} \\
\dot{\forall}\end{array}$ & $\begin{array}{l}\infty \\
\text { r? } \\
0\end{array}$ & $\begin{array}{l}\text { ڤ̊ } \\
6\end{array}$ & $\begin{array}{l}\hat{b} \\
\infty \\
\infty\end{array}$ & $\begin{array}{l}1 \\
\infty \\
\infty\end{array}$ & $\frac{7}{6}$ & $\frac{9}{6}$ & $\begin{array}{l}8 \\
0 \\
0\end{array}$ & 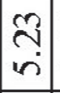 & $\begin{array}{l}8 \\
\stackrel{0}{0} \\
\dot{+}\end{array}$ & $\begin{array}{l}\stackrel{\partial}{0} \\
\dot{+}\end{array}$ & I & 1 & $\mathrm{I}$ & I & I \\
\hline \multirow{2}{*}{$\frac{\infty}{\stackrel{\infty}{0}}$} & $\otimes_{0}^{\circ}$ & 今. & $\begin{array}{c}0 \\
6 \\
0 \\
0\end{array}$ & $\begin{array}{l} \pm \\
\Xi \\
-1\end{array}$ & $\begin{array}{c}\stackrel{0}{1} \\
\dot{m}\end{array}$ & $\begin{array}{l}\tilde{\sigma} \\
\stackrel{0}{0}\end{array}$ & $\stackrel{\circ}{a}$ & $\begin{array}{l}\stackrel{\circ}{\prime} \\
\stackrel{i}{i}\end{array}$ & $\left|\begin{array}{c}8 \\
\dot{m} \\
\dot{m}\end{array}\right|$ & $\stackrel{\infty}{\Omega}$ & 弪 & $\tilde{n}$ & $\stackrel{n}{m}$ & ㄱ. & $\mid \begin{array}{l}0 \\
0 \\
0\end{array}$ & $\begin{array}{l}m \\
0\end{array}$ & - & \\
\hline & $\check{\Delta}$ & नि & & $\begin{array}{c}7 \\
3 \\
0\end{array} \mid$ & $\hat{\jmath}$ & $\begin{array}{l}\widehat{\sigma} \\
\infty \\
\infty\end{array}$ & $\frac{m}{\infty}$ & $\stackrel{2}{8}$ & $\frac{7}{6}$ & $\begin{array}{l}\mathscr{0} \\
\stackrel{0}{0} \\
\stackrel{0}{0}\end{array}$ & $\begin{array}{l}\stackrel{2}{i} \\
i n\end{array}$ & $\begin{array}{l}\vec{J} \\
\dot{f}\end{array}$ & 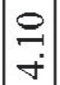 & I & $\mathrm{I}$ & $\mathrm{I}$ & I & I \\
\hline \multirow{2}{*}{$\frac{r}{\frac{\sigma}{0}}$} & $\delta_{0}^{\circ}$ & $\stackrel{ }{\stackrel{0}{-}}$ & $\begin{array}{c}8 \\
\infty \\
\vdots \\
0\end{array}$ & $\begin{array}{l}\mathscr{2} \\
\stackrel{0}{0}\end{array}$ & $\begin{array}{c}\vec{\sigma} \\
\dot{m}\end{array}$ & $\begin{array}{l}2 \\
0\end{array}$ & $\left|\begin{array}{l}\infty \\
i\end{array}\right|$ & $\stackrel{\vec{m}}{\mathrm{r}}$ & $\begin{array}{l}\stackrel{2}{\alpha} \\
\mathrm{i}\end{array}$ & $\Omega$ & {$\left[\begin{array}{c}n \\
n \\
\hdashline \\
1\end{array}\right]$} & $\begin{array}{l}\infty \\
\infty \\
\stackrel{\circ}{0}\end{array}$ & $\stackrel{\mathscr{P}}{-}$ & $\stackrel{n}{0}$ & $=$ & $\left|\begin{array}{c}\infty \\
2 \\
0\end{array}\right|$ & $\begin{array}{c}0 \\
-1 \\
\sim\end{array}$ & $\begin{array}{l}3 \\
8 \\
0 \\
0\end{array}$ \\
\hline & $\stackrel{0}{0}$ & 文 & $\begin{array}{l}n \\
\dot{r} \\
\dot{*}\end{array}$ & $\begin{array}{l}n \\
? \\
6\end{array}$ & $\begin{array}{l} \pm \\
0 \\
0\end{array}$ & 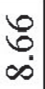 & $\mid \begin{array}{c} \pm \\
\infty \\
\infty\end{array}$ & $\begin{array}{l}n \\
6\end{array}$ & \begin{tabular}{c}
1 \\
\hdashline \\
0
\end{tabular} & $\begin{array}{l}\hat{\sigma} \\
0\end{array}$ & 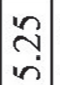 & 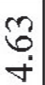 & $\begin{array}{l}\stackrel{0}{1} \\
\dot{\sigma}\end{array} \mid$ & I & I & $\mathrm{I}$ & I & 1 \\
\hline \multirow{2}{*}{$\frac{0}{\frac{0}{0}}$} & $\stackrel{\circ}{\circ}$ & $\mid \begin{array}{c}\tilde{b} \\
0\end{array}$ & $\begin{array}{l}n \\
\vdots \\
0 \\
0\end{array}$ & $\underset{8}{8}$ & 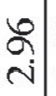 & $\stackrel{I}{g}$ & $\begin{array}{l}\bar{a} \\
\hat{\lambda}\end{array}$ & 2 & $\left|\begin{array}{c}n \\
\infty \\
i \\
\sim\end{array}\right|$ & $\stackrel{\infty}{-}$ & 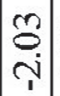 & సָ & 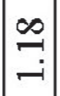 & & $\begin{array}{l}f \\
\dot{0}\end{array}$ & 8 & $\begin{array}{c}\mathcal{N} \\
\stackrel{\mathcal{N}}{\mathrm{N}}\end{array} \mid$ & 宫 \\
\hline & $\stackrel{0}{0}$ & กิ & $\begin{array}{r}r \\
\dot{r}\end{array}$ & $\begin{array}{c}+ \\
\text { ? } \\
0\end{array}$ & $\begin{array}{l} \pm \\
0 \\
0\end{array}$ & $\begin{array}{l}3 \\
\infty \\
\infty \\
\infty\end{array}$ & $\frac{m}{\infty}$ & $\begin{array}{l}9 \\
6 \\
0\end{array}$ & $\frac{m}{6}$ & $\begin{array}{l}\hat{\sigma} \\
0 \\
0\end{array}$ & $\begin{array}{l}\text { Ny } \\
\text { in }\end{array}$ & $\begin{array}{l}\overline{6} \\
\dot{+}\end{array}$ & $\begin{array}{l}\exists \\
\dot{\gamma}\end{array}$ & I & I & I & I & $\mathrm{I}$ \\
\hline \multirow{2}{*}{$\frac{n}{\frac{0}{0}}$} & \&̊ & $\begin{array}{l}\infty \\
\cdots \\
0\end{array}$ & $\begin{array}{c}0 \\
\stackrel{m}{0} \\
0\end{array}$ & 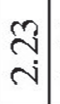 & $\begin{array}{c}0 \\
\dot{q} \\
\dot{r}\end{array}$ & $\stackrel{\infty}{n}$ & $\begin{array}{c}\vec{\sigma} \\
\dot{r}\end{array} \mid$ & $\overrightarrow{\mathrm{N}}$ & $\mid \begin{array}{l}\stackrel{\alpha}{\Omega} \\
\mathrm{\sim}\end{array}$ & $\Omega$ & $\frac{n}{n}$ & $\begin{array}{l}6 \\
0 \\
0\end{array}$ & 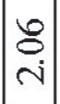 & $\frac{m}{0}$ & $\stackrel{3}{0}$ & $\begin{array}{l}\bar{a} \\
0\end{array}$ & $\begin{array}{c}\text { Nิ } \\
\text { ป }\end{array}$ & $\begin{array}{l}0 \\
0 \\
0 \\
0 \\
0\end{array}$ \\
\hline & $\stackrel{0}{0}$ & $\vec{\nabla}$ & $\begin{array}{c}E \\
+ \\
\end{array}$ & ㄱ. & $\begin{array}{l}\bar{a} \\
0\end{array}$ & $\begin{array}{l}\stackrel{2}{n} \\
\infty\end{array}$ & \begin{tabular}{|c}
5 \\
$\infty$ \\
$\infty$
\end{tabular} & $\begin{array}{l}\stackrel{0}{6} \\
6\end{array}$ & \begin{tabular}{c}
1 \\
\hdashline \\
6
\end{tabular} & $\begin{array}{l}6 \\
6 \\
0\end{array}$ & \begin{tabular}{|c|}
$n$ \\
$n$ \\
$n$
\end{tabular} & $\begin{array}{l}\dot{U} \\
\dot{+} \\
\end{array}$ & $\begin{array}{l}5 \\
\dot{+} \\
\end{array}$ & I & I & I & I & I \\
\hline \multirow{2}{*}{$\frac{\nabla}{\frac{\sigma}{g}}$} & $\stackrel{\circ}{0}$ & $\stackrel{n}{n}$ & 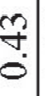 & $\stackrel{n}{2}$ & $\begin{array}{c}\text { ণ̃ } \\
\text { ๓r}\end{array}$ & $\overline{-}$ & $\left|\begin{array}{c}7 \\
\hdashline \dot{r} \\
\dot{n}\end{array}\right|$ & $\begin{array}{r}\stackrel{\oplus}{i} \\
\stackrel{\sim}{ }\end{array}$ & $\mid \begin{array}{l}\vec{v} \\
\text { ì }\end{array}$ & $\underline{F}$ & $\begin{array}{l}m \\
\vec{i} \\
\sim\end{array} \mid$ & $\begin{array}{l}\stackrel{8}{0} \\
\stackrel{0}{0}\end{array}$ & $\begin{array}{l}\hat{\sigma} \\
\dot{j}\end{array} \mid$ & $\stackrel{\infty}{\infty}$ & ?n & - & 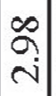 & $\frac{5}{5}$ \\
\hline & 0 & 守 & $\begin{array}{l}\hat{F} \\
\dot{\nabla}\end{array}$ & 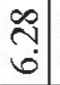 & $\begin{array}{c}\widehat{\sigma} \\
\sigma\end{array}$ & 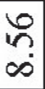 & $=\frac{F}{\infty}$ & $\begin{array}{l}2 \\
6 \\
6\end{array}$ & $\frac{m}{6}$ & $\begin{array}{l}\stackrel{\infty}{0} \\
\qquad \\
0\end{array}$ & $\begin{array}{l}\infty \\
i \\
n\end{array} \mid$ & $\begin{array}{l}\mathbb{J} \\
\dot{f}\end{array}$ & $\begin{array}{l}2 \\
\dot{+}\end{array}$ & I & I & $\mathrm{I}$ & I & I \\
\hline \multirow{2}{*}{$\frac{m}{\frac{\pi}{0}}$} & $\delta_{0}^{\circ}$ & $\begin{array}{c}0 \\
0 \\
0\end{array}$ & $\begin{array}{l}0 \\
\vdots \\
\vdots \\
i \\
1\end{array}$ & $\begin{array}{c}8 \\
8 \\
\dot{r}\end{array}$ & $\begin{array}{l}a \\
\mathrm{~m}\end{array}$ & $\begin{array}{l}n \\
m \\
m\end{array}$ & $\begin{array}{c}\text { ले } \\
\dot{+}\end{array}$ & $\stackrel{m}{q}$ & $\begin{array}{c}\vec{f} \\
\mathrm{i}\end{array}$ & ' & $\left|\begin{array}{c}\hat{\sigma} \\
\hat{i}\end{array}\right|$ & $\begin{array}{l}\infty \\
0 \\
0\end{array}$ & $\mid \begin{array}{l}0 \\
\stackrel{0}{-}\end{array}$ & $\stackrel{?}{\stackrel{9}{\circ}}$ & $\mid \begin{array}{l}0 \\
0 \\
0\end{array}$ & 근 & 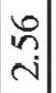 & $\begin{array}{l}\infty \\
\Omega \\
0\end{array}$ \\
\hline & $\check{0}$ & 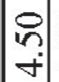 & $\begin{array}{c}\mathbb{N} \\
\infty \\
\forall\end{array}$ & तु & $\begin{array}{c}\infty \\
6 \\
6\end{array}$ & $\begin{array}{l}\underset{\infty}{+} \\
\dot{\infty}\end{array}$ & $\left|\begin{array}{c}\sigma \\
\infty \\
\infty\end{array}\right|$ & $\begin{array}{l}8 \\
6 \\
6\end{array}$ & $\begin{array}{c}n \\
6 \\
6\end{array}$ & $\begin{array}{l}8 \\
\stackrel{8}{0} \\
\text {. }\end{array}$ & 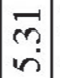 & $\begin{array}{l}\vec{J} \\
\dot{+}\end{array}$ & $\begin{array}{l}8 \\
\dot{+}\end{array}$ & I & I & I & I & $\mathrm{I}$ \\
\hline \multirow{2}{*}{$\frac{\frac{N}{0}}{\frac{D}{\delta}}$} & ò & กิ & 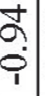 & $\begin{array}{l}\stackrel{\sim}{\sim} \\
\stackrel{\sim}{*}\end{array}$ & $\begin{array}{l}2 \\
\dot{r} \\
\dot{r}\end{array}$ & $\underset{\dot{r}}{\mathscr{\theta}}$ & 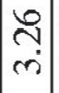 & $\underset{-}{\vec{\gamma}}$ & $\underline{\Omega}$ & 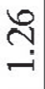 & $\begin{array}{c}0 \\
\tilde{m} \\
\mathrm{i}\end{array}$ & $\begin{array}{l}2 \\
\dot{p} \\
\end{array}$ & $\left|\begin{array}{c}\infty \\
\dot{r} \\
\dot{n}\end{array}\right|$ & $\stackrel{\infty}{\infty}$ & 을 & $\stackrel{n}{\stackrel{n}{g}}$ & $\hat{⿵}$ & $\frac{0}{2}$ \\
\hline & $\stackrel{0}{0}$ & $\stackrel{N}{\sim}$ & $\begin{array}{l}3 \\
\infty \\
+ \\
+\end{array}$ & 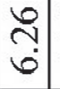 & ఫे & $\begin{array}{l}0 \\
+ \\
\infty \\
\infty\end{array}$ & $\left|\begin{array}{c}0 \\
\infty \\
\infty\end{array}\right|$ & $\begin{array}{l}\infty \\
\infty \\
0\end{array}$ & $\left|\begin{array}{c}\infty \\
-\vdots \\
0\end{array}\right|$ & $\overrightarrow{0}$ & $\begin{array}{l}\stackrel{2}{ } \\
\text { in }\end{array}$ & $\begin{array}{l}\infty \\
0 \\
\dot{+}\end{array}$ & $\mid \begin{array}{l}\mathcal{O} \\
\dot{f}\end{array}$ & I & I & 1 & I & 1 \\
\hline \multirow{2}{*}{$\frac{7}{\bar{c}}$} & of & 吉 & $\begin{array}{l}0 \\
6 \\
0 \\
1 \\
1\end{array}$ & $\begin{array}{l}\vec{J} \\
\dot{\sim} \\
\dot{1}\end{array}$ & $\begin{array}{l}\AA \\
\text { mे }\end{array}$ & $\stackrel{n}{n}$ & $\left|\begin{array}{c}\stackrel{0}{2} \\
\dot{r}\end{array}\right|$ & $\begin{array}{l}\stackrel{g}{\mathrm{~d}} \\
\stackrel{\mathrm{j}}{ }\end{array}$ & $\begin{array}{c}\hat{2} \\
\text { ì }\end{array}$ & $\stackrel{P}{i}$ & 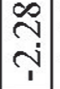 & $\begin{array}{l}\bar{Z} \\
\\
\end{array}$ & $\mid \begin{array}{l}\tilde{m} \\
\tilde{i}\end{array}$ & & $\stackrel{\Xi}{\stackrel{\Xi}{-}}$ & $\mid \begin{array}{c}5 \\
-i\end{array}$ & $\begin{array}{c}\stackrel{\sim}{c} \\
\dot{m}\end{array} \mid$ & $\frac{\tilde{2}}{2}$ \\
\hline & $\ddot{0}$ & 文 & $\begin{array}{l}\mathbb{D} \\
+ \\
+\end{array}$ & 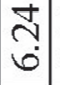 & $\begin{array}{l}\infty \\
\infty \\
0 \\
6\end{array}$ & $\begin{array}{l}\mathcal{F} \\
\infty\end{array}$ & $\left|\begin{array}{c}8 \\
\infty \\
\infty\end{array}\right|$ & $\frac{F}{6}$ & $\begin{array}{c}0 \\
-1 \\
0\end{array}$ & $\begin{array}{l}0 \\
0 \\
0 \\
0\end{array}$ & $\begin{array}{l}2 \\
\text { in }\end{array}$ & $\begin{array}{l}6 \\
\dot{\sigma}\end{array}$ & $\left|\begin{array}{l}8 \\
\dot{\gamma} \\
\dot{+}\end{array}\right|$ & I & I & $\mathrm{I}$ & I & I \\
\hline \multicolumn{2}{|c|}{$(9 I 0 Z-\angle 86 I) u G$} & in & $\begin{array}{l}2 \\
+ \\
+\end{array}$ & $\begin{array}{l}7 \\
7 \\
0\end{array}$ & $\underset{1}{\frac{6}{2}}$ & $\underset{\infty}{\stackrel{m}{\infty}}$ & $\left|\begin{array}{c}\infty \\
\infty \\
\infty\end{array}\right|$ & बై & $\mid \begin{array}{c}\overline{7} \\
\dot{6}\end{array}$ & $\begin{array}{l}\otimes \\
\varnothing \\
6\end{array}$ & $\frac{7}{18}$ & $\begin{array}{l}5 \\
+ \\
+\end{array}$ & $\begin{array}{l}0 \\
+ \\
+\end{array}$ & & & & & \\
\hline \multicolumn{2}{|c|}{ 4140\% } & & 节 & $\stackrel{\Xi}{\Sigma}$ & 荌 & $\stackrel{\mathrm{m}}{\mathrm{z}}$ & $\Xi$ & $\Xi$ & 密 & હे & 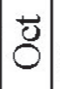 & z & $\mid \begin{array}{l}0 \\
\mathscr{\Xi} \\
0\end{array}$ & 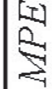 & $\mid \begin{array}{l}101 \\
09 \\
3\end{array}$ & $\mid \begin{array}{l}0 \\
\text { : }\end{array}$ & 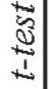 & $\begin{array}{l}\frac{1}{5} \\
2 \\
2\end{array}$ \\
\hline
\end{tabular}




\begin{tabular}{|c|c|c|c|c|c|c|c|c|c|c|c|c|c|c|c|c|c|c|}
\hline \multirow{2}{*}{$\begin{array}{l}\frac{ \pm}{\sigma} \\
\frac{\sigma}{\delta} \\
\frac{0}{2}\end{array}$} & $\stackrel{\circ}{\circ}$ & ฉి & $\begin{array}{l}2 \\
0\end{array}$ & $\stackrel{-}{-}$ & $\begin{array}{l} \pm \\
\stackrel{\mathrm{N}}{ }\end{array}$ & $\stackrel{\infty}{\infty}$ & $\left|\begin{array}{c}0 \\
\infty \\
ن\end{array}\right|$ & $\begin{array}{l}\infty \\
\stackrel{2}{\mathrm{i}} \\
\end{array}$ & $\begin{array}{l}+ \\
\infty \\
i\end{array}$ & $\begin{array}{l}0 \\
-i\end{array} \mid$ & $\begin{array}{l}8 \\
\stackrel{8}{1} \\
-1\end{array}$ & $\begin{array}{l} \pm \\
\vdots\end{array}$ & 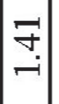 & $\stackrel{7}{0}$ & 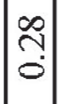 & ஓ. & $\left|\begin{array}{c}\infty \\
\stackrel{\sim}{\sim}\end{array}\right|$ & 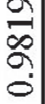 \\
\hline & $\stackrel{0}{0}$ & fे & $\begin{array}{l}\stackrel{2}{+} \\
+\end{array}$ & 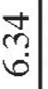 & ڤ̊. & $\begin{array}{l}0 \\
0 \\
\infty\end{array}$ & $\begin{array}{l} \pm \\
\infty \\
\infty\end{array} \mid$ & $\frac{7}{6}$ & $\begin{array}{c}m \\
0 \\
0\end{array}$ & $\left|\begin{array}{l}0 \\
0 \\
6\end{array}\right|$ & 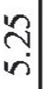 & $\begin{array}{c}3 \\
+ \\
+\end{array}$ & $\begin{array}{l}\stackrel{0}{1} \\
\dot{+}\end{array}$ & I & 1 & $\mathrm{I}$ & $\mathrm{I}$ & I \\
\hline \multirow{2}{*}{ 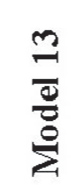 } & $i_{0}^{\circ}$ & $\underset{-}{\stackrel{N}{-1}}$ & $\begin{array}{l}\infty \\
\stackrel{\infty}{\circ}\end{array}$ & $\stackrel{n}{0}$ & $\begin{array}{l}\stackrel{0}{2} \\
i\end{array}$ & $\stackrel{m}{\circ}$ & in & 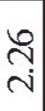 & $\begin{array}{l}R \\
i \\
ن\end{array}$ & $\mid \begin{array}{l}* \\
-i\end{array}$ & \begin{tabular}{c}
$\stackrel{ \pm}{\sim}$ \\
\hdashline \\
\hdashline
\end{tabular} & $\stackrel{\sim}{\circ}$ & $\stackrel{2}{2}$ & $\stackrel{0}{\circ}$ & 宇 & $\begin{array}{l}\Omega \\
\vdots \\
\delta\end{array}$ & \begin{tabular}{l|}
$\mid$ \\
6 \\
$ن$ \\
$ن$
\end{tabular} & 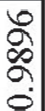 \\
\hline & $\check{0}$ & $\begin{array}{l}\text { of } \\
+ \\
+\end{array}$ & $\begin{array}{l}r \\
\dot{\sigma}\end{array}$ & (ָ) & 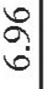 & $\begin{array}{l}\hat{\sigma} \\
\infty\end{array}$ & $\left|\begin{array}{c}0 \\
-\infty \\
\infty\end{array}\right|$ & $\stackrel{゚}{6}$ & $\begin{array}{l} \pm \\
\stackrel{0}{0}\end{array}$ & $\left|\begin{array}{l}\hat{0} \\
6 \\
0\end{array}\right|$ & $\begin{array}{l}0 \\
\qquad 1 \\
i\end{array}$ & 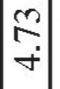 & $\mid \begin{array}{l}\infty \\
\stackrel{+}{+} \\
\end{array}$ & I & 1 & I & 1 & 1 \\
\hline \multirow{2}{*}{$\begin{array}{l}\text { I } \\
\text { d } \\
\frac{0}{2}\end{array}$} & o̊ & $\stackrel{m}{0}$ & $\begin{array}{l}\stackrel{0}{\circ} \\
\circ\end{array}$ & $\begin{array}{l}-\infty \\
0\end{array}$ & $\begin{array}{l} \pm \\
\stackrel{\mathrm{N}}{ }\end{array}$ & $\begin{array}{l}\sigma \\
0\end{array}$ & $\mid \begin{array}{l}F \\
i\end{array}$ & $\stackrel{\text { co }}{\mathrm{i}}$ & $\left|\begin{array}{l}\infty \\
\infty \\
i\end{array}\right|$ & $\mid \begin{array}{c}\infty \\
-i\end{array}$ & \begin{tabular}{l}
0 \\
\hdashline \\
-1
\end{tabular} & $\begin{array}{l}0 \\
0 \\
0\end{array}$ & הָ & $\stackrel{2}{0}$ & $=$ & $\left|\begin{array}{c}\infty \\
0 \\
0\end{array}\right|$ & $\left|\begin{array}{c}\infty \\
\infty \\
-i \\
-1\end{array}\right|$ & ळ \\
\hline & $\stackrel{0}{0}$ & ही & $\stackrel{2}{r}$ & 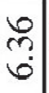 & مُ & $\mid \begin{array}{l}\infty \\
\infty \\
\infty\end{array}$ & $\begin{array}{l}\because \\
\infty \\
\infty\end{array} \mid$ & $\stackrel{7}{8}$ & $\frac{7}{6}$ & $\left|\begin{array}{c}6 \\
6 \\
0\end{array}\right|$ & 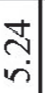 & $\begin{array}{l}\dot{J} \\
\dot{+}\end{array} \mid$ & $\begin{array}{l}\exists \\
\dot{+}\end{array}$ & I & 1 & I & 1 & 1 \\
\hline \multirow{2}{*}{$\begin{array}{l}= \\
\overline{0} \\
\frac{0}{\delta} \\
\Sigma\end{array}$} & o̊ & $\stackrel{2}{2}$ & $\begin{array}{c}5 \\
0\end{array}$ & $\begin{array}{l}\vec{\infty} \\
\dot{0}\end{array}$ & $\begin{array}{l}\stackrel{\partial}{0} \\
\text { in }\end{array}$ & f. & $\left|\begin{array}{l}\infty \\
\stackrel{0}{ } \\
\dot{\sim}\end{array}\right|$ & $\begin{array}{l}\stackrel{\partial}{0} \\
\stackrel{i}{*}\end{array}$ & $\begin{array}{l}-\infty \\
\dot{\sim}\end{array}$ & $\stackrel{F}{-i}$ & \begin{tabular}{c}
$\stackrel{\infty}{\sim}$ \\
\hdashline \\
\hdashline
\end{tabular} & ले & m. & ñ & $\frac{2}{0}$ & $\begin{array}{l}\infty \\
0 \\
0\end{array}$ & 공 & $\begin{array}{l}0 \\
\infty \\
0 \\
0\end{array}$ \\
\hline & $\stackrel{0}{0}$ & กी & $\begin{array}{l}r \\
\dot{f} \\
\end{array}$ & 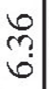 & $\begin{array}{l}\stackrel{2}{2} \\
\vdots\end{array}$ & $\begin{array}{c}0 \\
0 \\
\infty\end{array}$ & $\begin{array}{l}\because \\
\infty \\
\infty\end{array} \mid$ & $\underset{8}{7}$ & $\frac{m}{6}$ & $\left|\begin{array}{c}0 \\
0 \\
0 \\
0\end{array}\right|$ & $\begin{array}{l}3 \\
\\
\text { Int }\end{array}$ & $\left|\begin{array}{l}2 \\
\dot{+}\end{array}\right|$ & 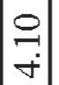 & I & I & I & I & I \\
\hline \multicolumn{2}{|c|}{$(9 I 0 z-\angle 86 I) u G$} & ?ִ & 9 & $\overrightarrow{7}$ & 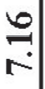 & $\begin{array}{l}n \\
\infty \\
\infty\end{array}$ & $\left|\begin{array}{c}\infty \\
\infty \\
\infty\end{array}\right|$ & ఫิ & ⿹ें & $\left|\begin{array}{c}\infty \\
6 \\
\emptyset\end{array}\right|$ & 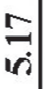 & $\begin{array}{l}\mathbf{6} \\
+ \\
+\end{array}$ & $\mid \begin{array}{l}0 \\
\dot{7}\end{array}$ & & & & & \\
\hline \multicolumn{2}{|c|}{ чриој } & & $\begin{array}{l}0 \\
0 \\
0\end{array}$ & $\stackrel{\vec{\pi}}{\Sigma}$ & 节 & $\sum_{i}^{\frac{\pi}{\Delta}}$ & 思 & $\Xi$ & 隹 & ڤ్ & $\overrightarrow{0}$ & $\begin{array}{l}0 \\
z \\
z\end{array}$ & $\begin{array}{l}0 \\
\varnothing\end{array}$ & 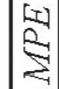 & $\left|\begin{array}{l|}1 \\
0 \\
0 \\
3\end{array}\right|$ & 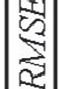 & 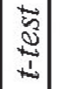 & 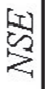 \\
\hline
\end{tabular}




\begin{tabular}{|c|c|c|c|c|c|c|c|c|c|c|c|c|c|c|c|c|c|c|}
\hline \multirow{2}{*}{$\frac{e}{\frac{e}{0}}$} & $\stackrel{\circ}{\circ}$ & 이 & $\frac{\circ}{\circ}$ & $\begin{array}{l}\sigma \\
\dot{0}\end{array}$ & $\begin{array}{l}\infty \\
\infty \\
i\end{array}$ & \begin{tabular}{l}
$n$ \\
\hdashline \\
0
\end{tabular} & $\left|\begin{array}{l}\tilde{\sigma} \\
ن \\
ن\end{array}\right|$ & $\begin{array}{l}\infty \\
\stackrel{\sim}{\sim}\end{array}$ & $\stackrel{r}{i}$ & 足 & \begin{tabular}{c}
$m$ \\
\\
\hdashline
\end{tabular} & $\begin{array}{c}m \\
\tilde{o} \\
0\end{array}$ & $\mid \begin{array}{c}\infty \\
n \\
-i\end{array}$ & $\stackrel{m}{\circ}$ & $\stackrel{1}{0}$ & $\begin{array}{l}- \\
\infty \\
\dot{0}\end{array}$ & & \\
\hline & $\stackrel{\circ}{0}$ & $\stackrel{m}{\partial}$ & $\begin{array}{l}8 \\
\text { i. }\end{array}$ & $\begin{array}{l}n \\
\sim\end{array}$ & $\frac{\pi}{2}$ & $\stackrel{n}{\infty}$ & $\begin{array}{l}6 \\
\infty \\
\infty\end{array} \mid$ & $\frac{2}{2}$ & $\stackrel{8}{-}$ & 菅 & $\begin{array}{l}\nexists \\
\text { )े }\end{array}$ & $\begin{array}{l}\vec{m} \\
n \\
\end{array}$ & 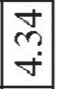 & 1 & $\mathrm{I}$ & 1 & I & 1 \\
\hline \multirow{2}{*}{$\frac{a}{\frac{0}{0}}$} & $\stackrel{\circ}{\circ}$ & 今. & $\begin{array}{l}\stackrel{0}{0} \\
0\end{array}$ & $\begin{array}{l}\mathbb{\sigma} \\
0 \\
0\end{array}$ & 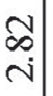 & $\begin{array}{l}\infty \\
+ \\
0 \\
0\end{array}$ & $\begin{array}{l}8 \\
i \\
ن\end{array} \mid$ & $\stackrel{n}{i}$ & $\begin{array}{l}\infty \\
i \\
i\end{array}$ & $\stackrel{\text { g. }}{\text { - }}$ & શิ & 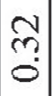 & $\left|\begin{array}{c}0 \\
+ \\
-i\end{array}\right|$ & $\frac{n}{8}$ & 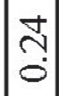 & & 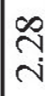 & $\begin{array}{l}2 \\
\infty \\
\Omega \\
\Omega\end{array}$ \\
\hline & $\check{0}$ & $\begin{array}{l}\Delta \\
\dot{f}\end{array}$ & $\begin{array}{l}8 \\
\dot{8} \\
\text { in }\end{array}$ & $\begin{array}{c}0 \\
-1 \\
\end{array}$ & $\frac{d}{\sim}$ & $\begin{array}{l}n \\
\infty \\
\infty\end{array}$ & $\left|\begin{array}{c}0 \\
\infty \\
\infty\end{array}\right|$ & ڤ్ & $\begin{array}{l}9 \\
\infty \\
-\infty\end{array}$ & חִ & సે. & $\begin{array}{l}\bar{m} \\
i n\end{array}$ & $\begin{array}{l}m \\
m \\
\dot{v}\end{array}$ & 1 & 1 & 1 & I & $\mathrm{I}$ \\
\hline \multirow{2}{*}{$\frac{\infty}{\frac{\infty}{0}}$} & $\stackrel{\circ}{\circ}$ & $\begin{array}{c}\infty \\
\infty \\
0\end{array}$ & $\frac{?}{\circ}$ & $\begin{array}{c}+ \\
\dot{0} \\
0\end{array}$ & $\begin{array}{l}\Omega \\
\text { ন }\end{array}$ & \begin{tabular}{l}
$N$ \\
\hdashline \\
0
\end{tabular} & $\left|\begin{array}{c}m \\
i\end{array}\right|$ & $\overrightarrow{\mathrm{N}}$ & $\begin{array}{l}\infty \\
\infty \\
\\
\end{array} \mid$ & $\stackrel{N}{2}$ & ণ্ণ & $\begin{array}{l}\stackrel{2}{2} \\
0\end{array}$ & 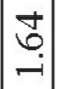 & $\stackrel{+}{0}$ & ?ָ. & & $\infty$ & হి \\
\hline & $\stackrel{0}{0}$ & $\frac{J}{\dot{\theta}}$ & 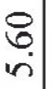 & $\begin{array}{l} \pm \\
⿱ 亠 䒑\end{array}$ & $\frac{m}{r}$ & $\begin{array}{l}0 \\
i \\
\infty \\
\infty\end{array}$ & $\left|\begin{array}{l}0 \\
0 \\
\infty\end{array}\right|$ & $\frac{\Omega}{r}$ & $\begin{array}{c}2 \\
\infty \\
-\infty\end{array}$ & 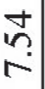 & 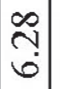 & $\begin{array}{c}1 \\
\tilde{n} \\
i n\end{array} \mid$ & 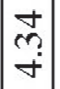 & 1 & $\mathrm{I}$ & $\mathrm{I}$ & I & 1 \\
\hline \multirow{2}{*}{$\frac{n}{\frac{0}{0}}$} & $\delta_{0}^{\circ}$ & $\hat{\partial}$ & $\begin{array}{c}\infty \\
0 \\
0\end{array}$ & $\begin{array}{c}\infty \\
\infty \\
0 \\
0\end{array}$ & $\begin{array}{l}2 \\
\mathrm{j}\end{array}$ & $\begin{array}{l}3 \\
6 \\
0 \\
0\end{array}$ & $\begin{array}{l}\mathfrak{m} \\
i\end{array} \mid$ & ત્. & $\begin{array}{l}\infty \\
\infty \\
i \\
\sim\end{array} \mid$ & $\begin{array}{l}+ \\
\infty \\
-1 \\
-1\end{array}$ & 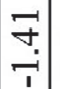 & $\begin{array}{l}\overline{6} \\
0 \\
0\end{array}$ & $\left|\begin{array}{c}\infty \\
\\
-\end{array}\right|$ & $\begin{array}{l}0 \\
? \\
0\end{array}$ & $\stackrel{?}{0}$ & $\begin{array}{c}\infty \\
0 \\
0\end{array}$ & $\frac{\circ}{i}$ & कू \\
\hline & $\stackrel{0}{0}$ & $\begin{array}{l}\vec{y} \\
\dot{\theta}\end{array}$ & $\begin{array}{l}8 \\
i \\
i\end{array}$ & $\underset{⿱ ㇒ 日}{2}$ & $\frac{m}{r}$ & $\begin{array}{l}n \\
\infty \\
\infty \\
\infty \\
0\end{array}$ & $\mid$ & 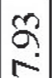 & $\begin{array}{l}\stackrel{2}{\infty} \\
\stackrel{-}{2}\end{array}$ & $\stackrel{n}{n}$ & 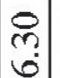 & in & $\stackrel{m}{m}$ & I & $\mathrm{I}$ & $\mathrm{I}$ & I & 1 \\
\hline \multirow{2}{*}{$\frac{0}{\frac{0}{0}}$} & ôे & $\mid \begin{array}{c}+ \\
0 \\
0\end{array}$ & $\frac{\mathfrak{2}}{0}$ & $\begin{array}{l} \pm \\
0\end{array}$ & $\begin{array}{l}\infty \\
\infty \\
ن\end{array}$ & $\begin{array}{l}\hat{\delta} \\
\dot{0}\end{array}$ & $\left|\begin{array}{c}\mathbb{N} \\
i\end{array}\right|$ & $\begin{array}{l}8 \\
i \\
ن\end{array}$ & $\begin{array}{l}8 \\
\dot{\infty} \\
\text { in }\end{array}$ & $F$ & $\mid$ & $\frac{0}{2}$ & $\left|\begin{array}{c}\infty \\
\end{array}\right|$ & F. & $\begin{array}{c}\infty \\
\tilde{n} \\
0\end{array} \mid$ & $\begin{array}{l}2 \\
0\end{array}$ & $\underset{\sim}{\stackrel{9}{i}}$ & $\begin{array}{l}1 \\
0 \\
\$ \\
0\end{array}$ \\
\hline & $\check{0}$ & $\stackrel{d}{a}$ & $\begin{array}{l}8 \\
0 \\
0\end{array}$ & $\stackrel{2}{2}$ & $\stackrel{?}{\sim}$ & $\begin{array}{c}\dot{t} \\
\infty \\
\infty\end{array}$ & $\left|\begin{array}{c}0 \\
0 \\
\infty \\
\infty\end{array}\right|$ & $\frac{2}{2}$ & $\stackrel{2}{\infty}$ & مُ & $\bar{n}$ & సి & $\mid$ & 1 & $\mathrm{I}$ & 1 & I & 1 \\
\hline \multirow{3}{*}{$\frac{n}{\frac{0}{0}}$} & & \$ & $n$ & 8 & 吉 & $\infty$ & $\vec{\approx}$ & 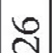 & 2 & న & in & 吉 & \pm & $\approx 2$ & I & ষ & $\approx$ & 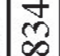 \\
\hline & & $0^{\circ}$ & $0^{\circ}$ & $-i$ & $\dot{r}$ & $-i$ & ri] & i & i & $-\dot{-i}$ & 7 & 0 & $-i$ & $0^{\circ}$ & $0^{\circ}$ & $0^{\circ}$ & i & å \\
\hline & $\ddot{0}$ & $\begin{array}{l} \pm \\
\dot{\sigma}\end{array}$ & $\begin{array}{c}\overline{6} \\
i\end{array}$ & $\begin{array}{l}8 \\
0 \\
-1\end{array}$ & $\frac{P}{2}$ & $\bar{n}$ & $\left|\begin{array}{c}\bar{b} \\
\infty\end{array}\right|$ & $\stackrel{m}{\Omega}$ & $\left|\begin{array}{c}\infty \\
\infty \\
\infty \\
\infty\end{array}\right|$ & $\stackrel{N}{n}$ & $\overline{\mid r}$ & ते & $\stackrel{m}{m}$ & 1 & $\mathrm{I}$ & 1 & I & 1 \\
\hline \multirow{3}{*}{$\frac{ \pm}{\frac{D}{0}}$} & & -1 & -1 & $n$ & $m$ & $n$ & 1 & -1 & 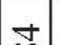 & $n$ & $m$ & $=1$ & 6 & 10 & 柾 & $N$ & 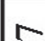 & 0 \\
\hline & & - & $\dot{0}$ & - & $\dot{r}$ & - & $i$ & $i$ & i & - & | & $\ddot{0}$ & $\mathrm{i}$ & $\stackrel{\circ}{\circ}$ & 0 & $\dot{0}$ & i & 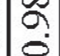 \\
\hline & $\stackrel{0}{0}$ & $\stackrel{4}{\pi}$ & $\overrightarrow{6}$ & 임. & $F$ & ஒे. & $\begin{array}{c}3 \\
6 \\
\infty\end{array}$ & $\frac{1}{2}$ & 官 & ñ. & 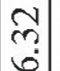 & సิ. & $\begin{array}{l}N \\
m\end{array}$ & 1 & 1 & 1 & I & 1 \\
\hline \multirow{3}{*}{$\frac{n}{\frac{0}{0}}$} & & 5 & \pm & 3 & $F$ & 아 & 8 & 5 & $\approx$ & $\approx$ & $\cong$ & 6 & 12 & 5 & I. & 18 & 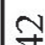 & $\bar{\infty}$ \\
\hline & & $\dot{0}$ & i' & ii & $\dot{r i}$ & $\dot{\sim}$ & $\dot{\nabla}$ & $-i$ & i & $-i$ & ง & $\ddot{0}$ & $-i$ & $0^{\circ}$ & 10 & $-i$ & $\dot{N}$ & 10 \\
\hline & $\stackrel{0}{0}$ & 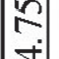 & 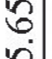 & $\stackrel{0}{\circ}$ & 官 & $\begin{array}{c}9 \\
+ \\
\infty \\
\infty\end{array}$ & $\mid \begin{array}{l} \pm \\
n \\
\infty \\
\infty\end{array}$ & 2 & 2 & ?ִ & $\mid$ & กิ & $\stackrel{m}{m}$ & 1 & 1 & 1 & I & 1 \\
\hline \multirow{3}{*}{$\frac{N}{\frac{N}{0}}$} & & $\stackrel{n}{7}$ & ris & \pm & 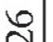 & $\vec{m}$ & $\exists$ & $\vec{\infty}$ & \pm & 2 & $\mathscr{\infty}$ & $\approx$ & $\approx$ & 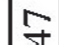 & $\because$ & $\infty$ & $\widetilde{N}$ & $\stackrel{\infty}{5}$ \\
\hline & & $0^{\circ}$ & î & i & $m$ & i & $\dot{r}$ & - & i & - & -i & 0 & i & 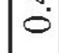 & $0^{\circ}$ & $-i$ & i & 0 \\
\hline & $\check{Q}$ & $\begin{array}{c}0 \\
\stackrel{0}{+}\end{array} \mid$ & $\begin{array}{l}0 \\
8 \\
i\end{array}$ & \begin{tabular}{c}
$\stackrel{\Xi}{0}$ \\
\hdashline
\end{tabular} & $\stackrel{P}{r}$ & $\begin{array}{c}\vec{\sigma} \\
\infty \\
\infty\end{array}$ & $\begin{array}{c}2 \\
n \\
\infty\end{array} \mid$ & 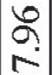 & $\begin{array}{l}\Omega \\
\Omega\end{array}$ & חִ & 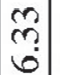 & $\begin{array}{l}n \\
n \\
n\end{array}$ & 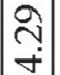 & 1 & $\mathrm{I}$ & 1 & I & 1 \\
\hline \multirow{2}{*}{$\frac{7}{\frac{\pi}{0}}$} & $\stackrel{\circ}{0}$ & $\stackrel{2}{0}$ & $\begin{array}{l}\mathbb{1} \\
0 \\
\dot{1}\end{array}$ & $\begin{array}{l}n \\
\infty \\
i\end{array}$ & $\begin{array}{c}\sigma \\
\text { r. }\end{array}$ & $\begin{array}{l}0 \\
\dot{0} \\
\text { r. }\end{array}$ & $\left|\begin{array}{l}\infty \\
\stackrel{0}{+}\end{array}\right|$ & $\vec{\sigma}$ & 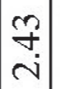 & త్ర & 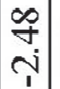 & $\begin{array}{l}\text { กิ } \\
\text { o. }\end{array}$ & बे & $\begin{array}{l}\infty \\
0 \\
0\end{array}$ & 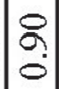 & $\underset{f}{-}$ & $\begin{array}{l}+ \\
\stackrel{\sim}{\sim}\end{array}$ & $\ddot{\infty}$ \\
\hline & $\stackrel{0}{0}$ & $\stackrel{m}{\nabla}$ & $\begin{array}{c}50 \\
\dot{n} \\
\end{array}$ & \begin{tabular}{l}
2 \\
\hdashline
\end{tabular} & 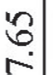 & 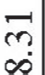 & $\left|\begin{array}{l} \pm \\
n \\
\infty\end{array}\right|$ & $\stackrel{2}{2}$ & $\begin{array}{l}\Omega \\
-\end{array}$ & ?ִ & $\begin{array}{l}0 \\
\text { ஸ̂. } \\
0\end{array}$ & mi & $\stackrel{\sim}{\tilde{m}}$ & 1 & 1 & 1 & I & 1 \\
\hline \multicolumn{2}{|c|}{$(9 I 0 z-\angle 86 I) u G$} & $\left|\begin{array}{c}\infty \\
⿱ \\
\nabla\end{array}\right|$ & $\begin{array}{c}\mathbb{6} \\
\text { (i) }\end{array}$ & ్ํ & مొ & $\begin{array}{l}\overline{6} \\
\infty \\
\infty\end{array}$ & $\left|\begin{array}{c}\Omega \\
\infty \\
\infty\end{array}\right|$ & $\underset{\infty}{\bar{\infty}}$ & $\frac{N}{\infty}$ & $\underset{\sim}{0}$ & \begin{tabular}{|c}
$\overrightarrow{0}$ \\
b.
\end{tabular} & $\begin{array}{c}\text { m. } \\
\text { in }\end{array}$ & $\begin{array}{l}\vec{y} \\
\dot{\nabla}\end{array}$ & & & & & \\
\hline \multicolumn{2}{|c|}{ чриоб } & & $\begin{array}{l}0 \\
0 \\
0\end{array}$ & $\sum^{ \pm}$ & 赵 & $\stackrel{\pi}{\Sigma_{1}^{*}}$ & $\Xi$ & $\Xi$ & $\stackrel{\infty}{\mathrm{Z}}$ & 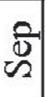 & $\overrightarrow{0}$ & $\begin{array}{l}3 \\
z \\
2\end{array}$ & $\mid \begin{array}{c}0 \\
\ddot{\Delta} \\
0\end{array}$ & $\frac{1}{1}$ & 뇜 & $\stackrel{a}{a}$ & 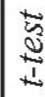 & 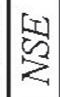 \\
\hline
\end{tabular}




\begin{tabular}{|c|c|c|c|c|c|c|c|c|c|c|c|c|c|c|c|c|c|c|}
\hline \multirow{2}{*}{$\begin{array}{l}\frac{ \pm}{ \pm} \\
\frac{\bar{d}}{8} \\
\frac{8}{2}\end{array}$} & ì & & & $\underset{-}{3}$ & $\begin{array}{c}3 \\
\dot{m}\end{array}$ & $\stackrel{8}{8}$ & $\overrightarrow{\widehat{\Omega}}$ & $\stackrel{2}{i}$ & 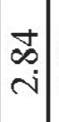 & $\begin{array}{c}\infty \\
-i\end{array}$ & בָ & $\stackrel{3}{6}$ & $\underset{\sim}{ \pm}$ & \begin{tabular}{l}
6 \\
0 \\
\hdashline
\end{tabular} & m? & ڤ̊ & $\begin{array}{c}n \\
\sim\end{array}$ & $\begin{array}{l}\infty \\
\infty \\
\Omega \\
0 \\
0\end{array}$ \\
\hline & ¿ & $\stackrel{9}{\circ}$ & $\begin{array}{l}8 \\
\dot{1} \\
\text { in }\end{array}$ & 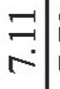 & $\stackrel{N}{\sim}$ & $\begin{array}{l}\stackrel{N}{\infty} \\
\infty \\
\infty\end{array}$ & $\begin{array}{c}\forall \\
\infty \\
\infty\end{array}$ & $\stackrel{2}{2}$ & $\begin{array}{c}\text { Dे } \\
\text { - }\end{array}$ & 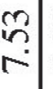 & 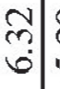 & $\begin{array}{l}\text { તે. } \\
\text { in }\end{array}$ & $\stackrel{m}{\square}$ & $\mathrm{I}$ & 1 & 1 & 1 & 1 \\
\hline \multirow{2}{*}{$\frac{n}{2}$} & $\stackrel{8}{\circ}$ & मे & 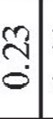 & $\begin{array}{l}\vec{y} \\
-\end{array}$ & $\begin{array}{c}\overrightarrow{0} \\
\dot{m}\end{array}$ & $\stackrel{m}{-}$ & $\begin{array}{l}\text { बे } \\
\text {. }\end{array}$ & $\begin{array}{l}\stackrel{?}{0} \\
i\end{array}$ & $\begin{array}{l}n \\
i \\
i\end{array}$ & 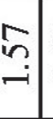 & $\begin{array}{l}\overrightarrow{6} \\
-1\end{array}$ & $\begin{array}{l}\infty \\
\overrightarrow{0} \\
0 \\
0\end{array}$ & $\begin{array}{l}\stackrel{\partial}{\vec{v}} \\
\vec{i}\end{array}$ & กิ & $\begin{array}{c}n \\
0 \\
0\end{array}$ & '. & $\begin{array}{l}\vec{b} \\
\stackrel{\mathrm{j}}{0}\end{array}$ & $\begin{array}{l}\text { §. } \\
\text { ఏ. } \\
\vdots \\
\vdots\end{array}$ \\
\hline & $\stackrel{\circ}{\Delta}$ & $\begin{array}{l}\text { J } \\
\dot{\theta}\end{array}$ & $\begin{array}{l}\hat{\sigma} \\
\dot{n}\end{array}$ & $\begin{array}{c}0 \\
\end{array}$ & $\begin{array}{c}\mathrm{N} \\
\stackrel{2}{2}\end{array}$ & $\underset{\infty}{\stackrel{9}{+}}$ & $\begin{array}{l}\tilde{b} \\
\infty\end{array}$ & 2 & $\bar{a}$ & $\stackrel{n}{n}$ & $\begin{array}{c}\text { సิ } \\
\text { | }\end{array}$ & $\begin{array}{l}\text { Yे } \\
\text { in }\end{array}$ & $\vec{m}$ & $\mathrm{I}$ & 1 & 1 & $\mathrm{I}$ & 1 \\
\hline \multirow{2}{*}{$\frac{\mathcal{J}}{\frac{\sigma}{\sigma}}$} & oे & ภे & $\begin{array}{l}\infty \\
\vdots \\
0\end{array}$ & ڤू. & $\begin{array}{l}\text { ડे } \\
\text { ஸे }\end{array}$ & $\stackrel{m}{\circ}$ & $\begin{array}{l}2 \\
i\end{array}$ & $\stackrel{ \pm}{\vec{i}}$ & $\begin{array}{l}\infty \\
\infty \\
i\end{array} \mid$ & 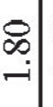 & \begin{tabular}{l} 
ஜ̂. \\
\hdashline \\
1
\end{tabular} & $\begin{array}{l}6 \\
0 \\
0\end{array}$ & ले & $\stackrel{m}{\circ}$ & $\begin{array}{c}\text { ్. } \\
\text { ○े }\end{array}$ & $\begin{array}{l}f \\
\dot{0} \\
\dot{0}\end{array}$ & ?] & $\begin{array}{l}+ \\
\text { ¿े } \\
\vdots \\
0\end{array}$ \\
\hline & $\stackrel{\circ}{\Delta}$ & 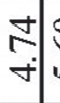 & $\begin{array}{l}8 \\
\text { in } \\
\text { in }\end{array}$ & $\stackrel{m}{\sim}$ & $\begin{array}{l}\stackrel{r}{r} \\
r\end{array}$ & $\begin{array}{l}n \\
\infty \\
\infty \\
\infty\end{array}$ & $\begin{array}{l}6 \\
0 \\
\infty\end{array}$ & ذ̊. & $\begin{array}{l}\dot{\infty} \\
\stackrel{2}{2}\end{array}$ & $\stackrel{m}{\stackrel{n}{r}}$ & $\begin{array}{c}\text { ల్లి } \\
\text { }\end{array}$ & $\begin{array}{l}\text { ते } \\
\text { in }\end{array}$ & $\stackrel{m}{m}$ & 1 & $\mathrm{I}$ & 1 & 1 & 1 \\
\hline \multirow{2}{*}{$\begin{array}{l}\exists \\
\overline{0} \\
\frac{0}{0} \\
\Sigma\end{array}$} & $\stackrel{8}{\circ}$ & $\hat{0}$ & $\begin{array}{c}\infty \\
\vdots \\
0\end{array}$ & $\vec{\sigma}$ & $\begin{array}{l}\vec{\alpha} \\
\text { iे }\end{array}$ & $\stackrel{6}{6}$ & $\begin{array}{l}\stackrel{0}{i} \\
\text { î }\end{array}$ & 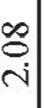 & {$\left[\begin{array}{c}\infty \\
\text { ஸे }\end{array} \mid\right.$} & $\stackrel{0}{\stackrel{0}{-}}$ & 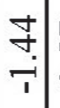 & 古 & $m$ & ñ? & 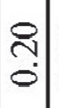 & $\begin{array}{c}\hat{\infty} \\
\dot{0} \\
\dot{0}\end{array}$ & $\tilde{3}$ & 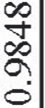 \\
\hline & $\stackrel{0}{0}$ & $\underset{\nabla}{\nabla}$ & $\begin{array}{l}8 \\
\stackrel{8}{0} \\
i\end{array}$ & $\frac{m}{r}$ & $\begin{array}{l}m \\
r\end{array}$ & $\begin{array}{l}n \\
\infty \\
\infty\end{array}$ & $\begin{array}{l}\hat{6} \\
\infty\end{array}$ & ذి & $\begin{array}{l}\stackrel{2}{\infty} \\
\text { مे }\end{array}$ & $\stackrel{n}{n}$ & 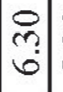 & $\begin{array}{l}\text { ஜ } \\
\text { nn }\end{array}$ & $\stackrel{n}{\sim}$ & 1 & $\mathrm{I}$ & 1 & 1 & $\mathrm{I}$ \\
\hline \multicolumn{2}{|c|}{$(9 I 0 z-\angle 86 I) u G$} & $\begin{array}{c}\infty \\
⿱ \\
+\end{array}$ & $\begin{array}{c}\mathbf{t} \\
\vdots \\
\dot{1}\end{array}$ & ్ㅗ & 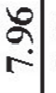 & $\begin{array}{l}\overline{6} \\
\infty\end{array}$ & $\begin{array}{l}\stackrel{్}{\infty} \\
\infty\end{array}$ & $=$ & $\underset{\infty}{7}$ & 6 & $\mid \begin{array}{c}\overrightarrow{0} \\
\text { s. }\end{array}$ & 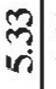 & $\begin{array}{l}F \\
+\end{array}$ & & & & & \\
\hline \multicolumn{2}{|c|}{ чриоб } & 氙 & & $\stackrel{ \pm}{ \pm}$ & 案 & 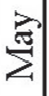 & $\Xi$ & $\Xi$ & 至 & 駋 & $\overrightarrow{0}$ & 艺 & $\begin{array}{c}8 \\
\varrho\end{array}$ & $\begin{array}{l}1 \\
0 \\
3 \\
z\end{array}$ & 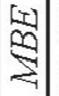 & 㝴 & 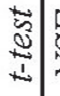 & $\begin{array}{l}\text { 空 } \\
2 \\
z\end{array}$ \\
\hline
\end{tabular}




\begin{tabular}{|c|c|c|c|c|c|c|c|c|c|c|c|c|c|c|c|}
\hline 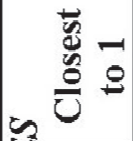 & $\begin{array}{l}\stackrel{2}{2} \\
\text { हे }\end{array}$ & $\widehat{\mathcal{\Xi}}$ & ڤั. & $\overparen{\Xi}$ & ڤ్ & $\stackrel{\Xi}{\Xi}$ & $\stackrel{2}{2}$ & $\stackrel{\overparen{త}}{\varrho}$ & $\begin{array}{l}\stackrel{2}{\circ} \\
\stackrel{\circ}{\circ}\end{array}$ & 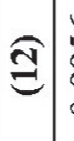 & 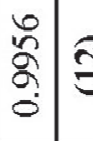 & $\overparen{\Xi} \mid \begin{array}{l}\tilde{g} \\
\dot{g} \\
0\end{array}$ & 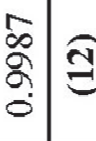 & 离 & $\overparen{\Xi}$ \\
\hline 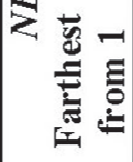 & \begin{tabular}{l}
\multirow{2}{\circ}{} \\
$\hat{\sigma}$ \\
0
\end{tabular} & $\approx$ & 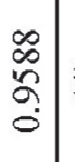 & $\overparen{Ð}$ & 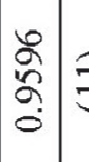 & $\Xi$ & $\begin{array}{l}\bar{b} \\
8 \\
0\end{array}$ & $\widehat{2}$ & $\begin{array}{l}\frac{m}{3} \\
\vdots \\
\sigma\end{array}$ & $\stackrel{Ð}{ }$ & $\begin{array}{c}\hat{N} \\
\stackrel{\sigma}{\circ}\end{array}$ & $\mathscr{f}$ & 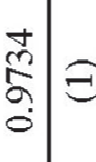 & $\frac{3}{0}$ & 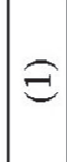 \\
\hline 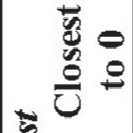 & $\begin{array}{l}\mathscr{8} \\
\dot{0}\end{array}$ & $\overparen{\Xi}$ & $\stackrel{n}{0}$ & $\underset{\exists}{\Xi}$ & $\stackrel{9}{0}$ & $\widetilde{\Xi}$ & $\overparen{\overbrace{}}$ & తิ & $\mid$ & $\widehat{\Xi}$ & $\stackrel{8}{8}$ & 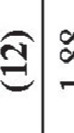 & $\begin{array}{l}\infty \\
\infty \\
- \\
-\end{array}$ & $\stackrel{n}{n}$ & $\overparen{\Xi}$ \\
\hline 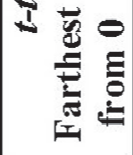 & $\frac{2}{2}$ & 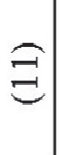 & $\begin{array}{l} \pm \\
i n\end{array}$ & $\overparen{d}$ & $\begin{array}{l}\infty \\
i \\
i\end{array}$ & $\widehat{\Xi}$ & $\underset{+\infty}{\infty}$ & $\widehat{\theta}$ & $\begin{array}{c}\hat{\sigma} \\
\dot{m}\end{array} \mid$ & 6 & $m$ & $\widehat{\mathcal{E}}$ & $\begin{array}{c}\stackrel{\ddots}{r} \\
\dot{m}\end{array}$ & i & $\widehat{\ominus}$ \\
\hline 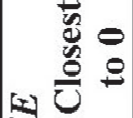 & $\stackrel{m}{m}$ & $\widehat{త}$ & $\stackrel{\overbrace{}}{\varrho}$ & $\widehat{త}$ & 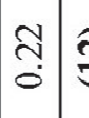 & $\widehat{త}$ & $\stackrel{n}{\tilde{\sigma}}$ & తิ) & $\stackrel{m}{o}$ & $\widehat{\mathcal{\Xi}}$ & $\stackrel{\infty}{\stackrel{\infty}{0}}=$ & $\widehat{\Xi}($ & $\begin{array}{l}\infty \\
0 \\
0\end{array}$ & 守 & $\overparen{త}$ \\
\hline 20 & $\stackrel{m}{-}$ & $\mathcal{\Xi}$ & $\vec{n}$ & $\mathcal{E}$ & 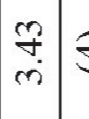 & $\mathcal{E}$ & $\begin{array}{l}\infty \\
\stackrel{\infty}{0} \\
\dot{r}\end{array}$ & $\widehat{d}$ & 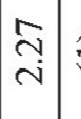 & $\Xi$ & $\stackrel{3}{0}$ & $\Xi$ & $\stackrel{0}{\stackrel{2}{-}}$ & 导 & 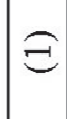 \\
\hline 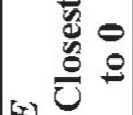 & $\begin{array}{l}8 \\
\\
\end{array}$ & $\overparen{\Xi}$ & $\begin{array}{l}\mathbf{0} \\
\stackrel{0}{i}\end{array}$ & $\stackrel{\overparen{త}}{\Xi}$ & $\stackrel{m}{\circ}$ & $\widehat{త}$ & ְे & తิత & $\begin{array}{l}\overrightarrow{0} \\
0\end{array}$ & త્ & $\overrightarrow{0}$ & $\stackrel{(\mathcal{Z}}{\exists}$ & $\vec{\Xi}: \overparen{\Xi}$ & $\stackrel{2}{3}$ & తิ \\
\hline 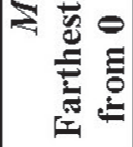 & $\bar{\sigma}$ & $\sqrt{2}$ & $\begin{array}{l}\infty \\
\stackrel{\infty}{i} \\
i\end{array}$ & $\overparen{d}$ & 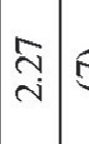 & $E$ & İ & $\widehat{d}$ & $\stackrel{8}{\circ}$ & $\widehat{త}$ & 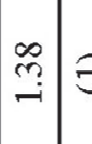 & 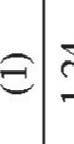 & 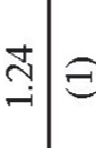 & @ి & 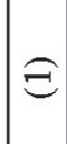 \\
\hline 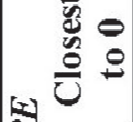 & $\stackrel{5}{\circ}$ & $\stackrel{\overbrace{}}{\Xi}$ & $\overrightarrow{0}$ & $\stackrel{\overparen{త}}{\Xi}$ & $\mid$ & $\overparen{త}$ & $\begin{array}{l}\stackrel{9}{+} \\
\stackrel{i}{i}\end{array}$ & $\stackrel{\overbrace ્ త}{\Xi}$ & $\begin{array}{l}\dot{0} \\
\dot{0} \\
\dot{i}\end{array}$ & $\underset{\Xi}{\beth}$ & $\stackrel{m}{\overrightarrow{0}}$ & $\stackrel{\overbrace{}}{\exists}$ & 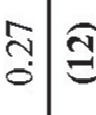 & $\frac{7}{3}$ & $\overparen{\Im}$ \\
\hline 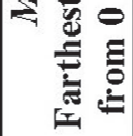 & $\begin{array}{l}\infty \\
\stackrel{0}{+} \\
\dot{f}\end{array}$ & 6 & $\begin{array}{c}7 \\
\text { in }\end{array}$ & $\overparen{త}$ & $\begin{array}{l}\text { बे } \\
\text { i }\end{array}$ & $\widehat{\Xi}$ & $\stackrel{n}{i}$ & $\widehat{\theta}$ & $\stackrel{\text { ra }}{\mathrm{N}}$ & $\mathcal{E}$ & $\stackrel{2}{r}$ & $\Xi$ & 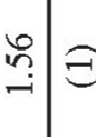 & $\stackrel{\infty}{\circ}$ & 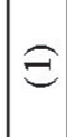 \\
\hline 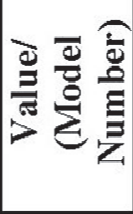 & $\frac{\mathscr{9}}{\frac{\pi}{7}}$ & 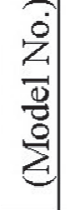 & $\frac{\mathscr{g}}{\overrightarrow{7}}$ & 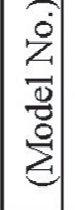 & $\frac{0}{5}$ & 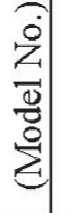 & $\frac{0}{3}$ & 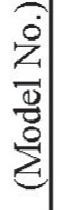 & 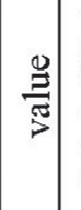 & 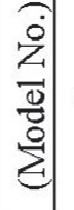 & $\frac{9}{3}$ & 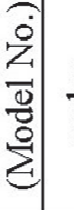 & $\frac{9}{3}$ & $\frac{\mathscr{g}}{3}$ & 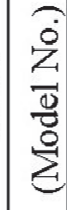 \\
\hline 竞 & 童 & & 苞 & 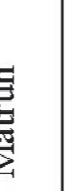 & 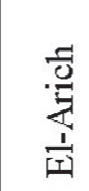 & & $\frac{\text { 当 }}{\frac{\pi}{E}}$ & & 逍 & & 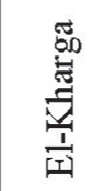 & & $\stackrel{\widetilde{Z}}{0}$ & & 营 \\
\hline
\end{tabular}




\section{Conclusions}

The most accurate empirical models that estimate diffuse solar radiation were collected from the literature to evaluate their applicability for estimate diffuse solar radiation over Egypt. The collected models were compared on the basis of the many statistical error tests; relative percentage error $(e \%)$, mean percentage error $(M P E)$, mean bias error $(M B D)$, root mean square error (RMSE), $t$-test, and Nash-Sutcliffe equation (NSE). According to the results, the Tarhan and Sarı model (Model 12) showed the best estimation of the diffuse solar radiation on a horizontal surface for all stations. Therefore, the Tarhan and Sar1 model (Model 12) is extremely recommended for predicting diffuse solar radiation at any location in Egypt.

\section{References}

Al-Mohamad A., 2004: Global, direct and diffuse solar radiation in Syria. Appl Energy 79 (2), 191-200. https://doi.org/10.1016/j.apenergy.2003.12.011

Almorox, J., and Hontoria C., 2004: Global solar radiation estimation using sunshine duration in Spain. Energy Conv. Manage 45, 1529-35. https://doi.org/10.1016/j.enconman.2003.08.022

Aras, H., Balli, O., Hepbasli, A., 2006: Estimating the horizontal diffuse solar radiation over the Central Anatolia region of Turkey. Energy Conv. Manage 47, 2240-2249. https://doi.org/10.1016/j.enconman.2005.11.024

Chen, R., Ersi, K., Yang, J., Lu, S., Zhao, W., 2004: Validation of five global radiation models with measured daily data in China. Energy Conv. Manage 45, 1759-69. https://doi.org/10.1016/j.enconman.2003.09.019

Darwish, M. A., and Taha, N. E., 2000: Estimation of diffuse radiation over the Arab World (Eastern Region). 5th conference-Meteorology \&Sustainable development 22-24 February 2000. Cairo, Egypt.

Driesse, A. and Thevenard, D. A., 2002: Test of Suehrcke's sunshine radiation relationship using a global data set. Sol. Energy 72, 167-75. https://doi.org/10.1016/S0038-092X(01)00082-2

Duffie, J. A. and Beckman, W. A., 1991: Solar engineering of thermal process. New York: Wiley.

El-Metwally, M., 2004: Simple new methods to estimate global solar radiation based on meteorological data in Egypt. Atmos. Res. 69, 217-239. https://doi.org/10.1016/j.atmosres.2003.09.002

El-Metwally, M., 2005: Sunshine and global solar radiation estimation at different sites in Egypt. $J$. Atmos. Solar-Terr. Phys. 67, 1331-1342. https://doi.org/10.1016/j.jastp.2005.04.004 https://doi.org/10.1080/01431161.2013.834393

El-Metwally, M. and Wald, L., 2013: Monthly means of daily solar irradiation over Egypt estimated from satellite database and various empirical formulae. Int. J. Remote Sens. 34, 8182-8198. https://doi.org/10.1080/01431161.2013.834393

El-Sebaii, A.A., and Trabea, A.A., 2003: Estimation of horizontal diffuse solar radiation in Egypt. Energy Conv. Manage. 44, 2471-2482. https://doi.org/10.1016/S0196-8904(03)00004-9

El-Sebaii, A.A., and Trabea, A.A., 2005: Estimation of Global Solar Radiation on Horizontal Surfaces over Egypt. Egypt. J. Solids. 28, 163-175.

El-Sebaii, A.A., Al-Hazmi, F. S., Al-Ghamdi, A.A., Yaghmour, S.J., 2010: Global, direct and diffuse solar radiation on horizontal and tilted surfaces in Jeddah, Saudi Arabia. Appl. Energy 87, 568-76. https://doi.org/10.1016/j.apenergy.2009.06.032

El-Shahawy, M.A., 1984: Estimation of daily global solar radiation. Bull. Fac. Sci. Cairo Univ. 52, 641-653.

El-Shazly, M.S., Abdelmageed, A.M. and El-Noubi, M., 1998: Solar radiation characteristics at Qena/ Egypt. Mausam 49, 59-70.

Gopinathan, K.K., 1988: Empirical correlations for diffuse solar radiation. Sol Energy 40, 369-70. https://doi.org/10.1016/0038-092X(88)90009-6

Hawas, M.M. and Muneer, T., 1984: Study of diffuse and global radiation characteristic in India. Energy Conv. Manage 24, 143-9. https://doi.org/10.1016/0196-8904(84)90026-8 
Ibrahim, S.M.A., 1985: Predicted and measured global solar radiation in Egypt. Sol Energy 35, 185-8. https://doi.org/10.1016/0038-092X(85)90009-X

Jamil, B. and Akhtar, N., 2017: Comparative analysis of diffuse solar radiation models based on sky clearness index and sunshine period for humid-subtropical climatic region of India: A case study. Renew. Sustain. Energy Rev. 78, 329-355. https://doi.org/10.1016/j.rser.2017.04.073

Khalil, S.A., and Shaffie, A. M., 2013: A comparative study of total, direct and diffuse solar irradiance by using different models on horizontal and inclined surfaces for Cairo, Egypt. Renew. Sustain. Energy Rev. 27, 853-63. https://doi.org/10.1016/j.rser.2013.06.038

Khalil, S.A., and Shaffie, A.M., 2016: Evaluation of transposition models of solar irradiance over Egypt. Renew. Sustain. Energy Rev. 66, 105-119. https://doi.org/10.1016/j.rser.2016.06.066

Kumar, R. and Umanand, L., 2005: Estimation of global radiation using clearness index model for sizing photovoltaic system. Renew Energy 30, 2221-2233. https://doi.org/10.1016/j.renene.2005.02.009

Li, D.H.W. and Lam, J.C., 2000: Solar heat gain factors and the implications for building designs in subtropical regions. Energy Build. 32, 47-55. https://doi.org/10.1016/S0378-7788(99)00035-3

Lu, Z., Piedrahita R.H., and Neto, C.D.S., 1998: Generation of daily and hourly solar radiation values for modeling water quality in aquaculture ponds. Trans ASAE 41, 1853-1859. https://doi.org/10.13031/2013.17323

Mediavilla, M.D., Miguel, A., and Bilbao, J., 2005: Measurement and comparison of diffuse solar irradiance models on inclined surfaces in Valladolid, Spain. Energy Conv. Manage 46, 2075-92. https://doi.org/10.1016/j.enconman.2004.10.023

Notton, G., Cristofari, C., and Muselli, M., Poggi, P., 2004: Calculation on an hourly basis of solar diffuse irradiations from global data for horizontal surfaces in Ajaccio. Energy Conv. Manage 45, 2849-2866. https://doi.org/10.1016/j.enconman.2004.01.003

Sabbagh, J.A., Sayigh, A.M., El Salam, E.M., 1977: Estimation of the total radiation from meteorological data. Solar Energy 19, 307-311. https://doi.org/10.1016/0038-092X(77)90075-5

Soares, J., Oliveira, A. P., Boznar, M. Z., Mlakar, P., Escobedo, J. F., and Machado, A.J., 2004: Modeling hourly diffuse solar radiation in the city of Sao Paulo using a neural network technique. Appl. Energy 79, 201-214. https://doi.org/10.1016/j.apenergy.2003.11.004

Stone, R.J., 1993: Improved statistical procedure for the evaluation of solar radiation models. Sol Energy 51, 289-91. https://doi.org/10.1016/0038-092X(93)90124-7

Tadros, M.T.Y., 2000: Uses of Sunshine Duration to Estimate the Global Solar Radiation over Eight Meteorological Stations in Egypt. Renew. Energy 21, 231-246. https://doi.org/10.1016/S0960-1481(00)00009-4

Tarhan, S. and Sarı, A., 2005: Model selection for global and diffuse radiation over the Central Black Sea (CBS) region of Turkey. Energy Conv. Manage 46, 605-613. https://doi.org/10.1016/j.enconman.2004.04.004

Tiba, C., 2001: Solar radiation in the Brazilian Northeast. Renew. Energy 22, 565-578. https://doi.org/10.1016/S0960-1481(00)00116-6

Togrul, I.T., and Togrul, H., 2002: Global solar radiation over Turkey: comparison of predicted and measured data. Renew. Energy 25, 55-67. https://doi.org/10.1016/S0960-1481(00)00197-X

Trabea, A., and Shaltout, M.A., 2000: Correlation of Global Solar Radiation with Meteorological Parameters over Egypt. Renew. Energy 21, 297-308. https://doi.org/10.1016/S0960-1481(99)00127-5

Tymvios, F.S., Jacovides, C.P., Michaelides, S.C., and Scouteli, C., 2005: Comparative study of Angstrom and artificial neural networks methodologies in estimating global solar radiation. Sol Energy 78, 752-62. https://doi.org/10.1016/j.solener.2004.09.007

Ulgen, K., and Hepbasli, A., 2002: Comparison of solar radiation correlations for Izmir, Turkey. Int. J. Energy Res. 26, 413-430. https://doi.org/10.1002/er.794

Ulgen, K., and Hepbasli, A., 2003: Comparison of diffuse fraction of daily and monthly global radiation for Izmir, Turkey. Energy Sour. 25, 637-649. https://doi.org/10.1080/00908310390212444

Ulgen, K., and Hepbasli, A., 2004: Solar radiation models. Part 2: Comparison and developing new models. Energy Sour. 26, 521-530. https://doi.org/10.1080/00908310490429704

Ulgen, K., and Hepbasli, A., 2009: Diffuse solar radiation estimation models for Turkey's big cities. Energy Conv. Manage 50, 149-56. https://doi.org/10.1016/j.enconman.2008.08.013

Wong, L.T., and Chow, W.K., 2001: Solar radiation model. Appl. Energy 69,191-224. https://doi.org/10.1016/S0306-2619(01)00012-5 